\title{
NONLINEAR DYNAMIC RESPONSE OF SEISMICALLY EXCITED RECTANGULAR CONCRETE LIQUID FILLED TANKS
}

\author{
By \\ Arash Farzin \\ B.Eng, Azad University Central Branch, Tehran, Iran, 2009 \\ A thesis \\ presented to Ryerson University \\ in partial fulfillment of the \\ requirements for the degree of \\ Master of Applied Science \\ in the program of \\ Civil Engineering
}

Toronto, ON, Canada, 2018

(C)Arash Farzin, 2018 


\section{DECLARATION}

I hereby declare that I am the sole author of this thesis. This is a true copy of the thesis, including any required final revisions, as accepted by my examiners.

I authorize Ryerson University to lend this thesis to other institutions or individuals for the purpose of scholarly research.

I further authorize Ryerson University to reproduce this thesis by photocopying or by other means, in total or in part, at the request of other institutions or individuals for the purpose of scholarly research.

I understand that my thesis may be made electronically available to the public.

Arash Farzin 


\title{
ABSTRACT \\ NONLINEAR DYNAMIC RESPONSE OF RECTANGULAR CONCRETE LIQUID TANKS IN THREE-DIMENSIONAL SPACE
}

\author{
Master of Applied Science, 2018 \\ Arash Farzin \\ Civil Engineering \\ Ryerson University
}

\begin{abstract}
The effect of nonlinearity on behaviour of rectangular concrete tanks partially filled with water is studied. The nonlinearity in the numerical modeling of the surface liquid sloshing performance and hydrodynamic pressure initiates from unknown boundary conditions of contained liquid volume. The nonlinear simulations are performed for Time-History seismic analysis using the finite element software ABAQUS/CAE. The nonlinear results are compared with linear analytical solutions and ACI 350.3-06 code. A Paramedic study is conducted to investigate the effect of tank plan dimension, frequency content of different seismic ground motions, nature of earthquake movements, and interaction of bi-directional component of earthquake on the maximum sloshing height of liquid. The results reveal that the nonlinearity is more significant in shallow tanks. Moreover, nonlinear hydrodynamic pressure distribution has no important difference with linear calculated pressure except for the surface sloshing pressure acting on the top of tanks. The linear ratio of depth of liquid to tank plan dimension used in ACI 350.3-06 formulation is found to be less accurate for calculating the maximum sloshing height of liquid.
\end{abstract}




\section{ACKNOWLEDGMENTS}

I wish to express my indeed respect to my supervisor Professor Reza Kianoush who guided me throughout my research from very early steps to the final ones with his extreme patience and knowledge. Without his review and criticism of the study, and his deep knowledge and experience on this problem this work could not be done. I always will be in debt to him for all unsparing kindness and support he showed me.

I wish to express my indeed gratitude to all of my colleagues in the Civil Engineering Department at Ryerson University; especially Dr. Mehdi Moslemi for his insight and guidance of the work had an important effect on this thesis.

I must convey my very profound gratitude to my wife Mehrnoosh for her constant support and sacrifice during my study. Moreover, I must convey my best regard to my mother for her unfailing kindness and support throughout my life.

Finally, I would like to thank Ryerson University for providing a flourishing academic environment. 


\section{Table of Contents}

Abstract

Acknowledgements $\quad$ iv

List of Tables $\quad$ vii

List of Figures viii

1 Introduction $\quad 1$

1.1 General overview 1

1.2 Methods of Analysis 4

1.3 Objective and Scope of Study 5

1.4 Thesis Layout 7

2 Literature Review $\quad 9$

2.1 Introduction 9

2.2 Seismic Damage to Liquid Storage Tanks 9

2.3 Previous Research Studies 12

2.4 Design Codes and Standards 16

3 Mathematical Background 2

$\begin{array}{lll}3.1 & \text { Introduction } & 26\end{array}$

3.2 Theories for Dynamic Analysis of Liquid Storage Tanks 27

3.3 Velocity Potential Theory Governing Equations 27

3.4 Solution of Equation for a Rectangular Tank 31

3.5 Analytical Derivation of Mechanical Model Parameters 35

$4 \quad$ Numerical Methods for Seismically Excited Liquid Storage Tanks 41

4.1 Introduction 41

4.2 Numerical Simulation Methods 41

4.2.1 Meshed-base Numerical Methods $\quad 41$

4.2.2 Lagrangian Method 42 
4.2.3 Smoothed Particle Hydrodynamics (SPH) 43

4.3 Governing Equations of liquid motion in the SPH method 46

4.3.1 Navier-Stokes Equation 46

$\begin{array}{lll}\text { 4.3.2 Continuity Equations } & 48\end{array}$

$\begin{array}{lll}\text { 4.3.3 Equation of State } & 48\end{array}$

4.3.4 Smoothing Length Calculation 49

4.3.5 Viscosity Modelling 49

$\begin{array}{lll}\text { 4.3.6 Kernel Function } & 50\end{array}$

4.3.7 Density Reinstallation Technique 51

4.3.8 Time increment Definition 51

4.3.9 Finite Element Conversion to SPH Particles 53

$5 \quad$ Nonlinear Sloshing Response of Rectangular Tanks: FEM Modelling, Results \& Discussion $\quad 54$

5.1 Introduction $\quad 54$

5.2 Application of Finite Element Program 54

5.3 Time History Analysis 61

5.3.1 Effect of Nonlinearity on Liquid Sloshing Height 63

5.3.2 Nonlinear Liquid Sloshing under Bi-directional Seismic Ground Motions $\quad 73$

5.3.3 Effect of Applying Bidirectional Seismic Component on Sloshing Response

5.3.4 Effect of Earthquake Frequency Content 87

5.3.5 Effect of Earthquake Frequency Content on Wave Interference 89

5.3.6 Nonlinear Hydrodynamic Pressure on the Walls 95

$6 \quad$ Summary, Conclusion, and Future Study $\quad 103$

$\begin{array}{lll}6.1 & \text { Summary } & 103\end{array}$

$\begin{array}{lll}6.2 & \text { Conclusions } & 104\end{array}$

6.3 Future Studies 106

$\begin{array}{ll}\text { References } & 107\end{array}$ 


\section{List of Tables}

$2.1 \quad$ Response modification factor $R \quad 19$

2.2 Importance factor $I \quad 19$

$5.1 \quad$ Selected tank models 57

5.2 Material properties of the FSI (Fluid-Structure Interaction) system 58

5.3 Maximum sloshing height from FE analysis 64

$\begin{array}{lll}5.4 & \text { Negative and positive maximum sloshing response } & 71\end{array}$

5.5 Comparison between ACI freeboard height and nonlinear FE sloshing 71

5.6 Comparison of nonlinear finite element and code estimations 72

5.7 Maximum liquid sloshing height under bi-directional seismic ground $\begin{array}{ll}\text { motions } & 75\end{array}$

$\begin{array}{lll}5.8 & \text { Normalized sloshing height values } & 76\end{array}$

5.9 Comparison of the sloshing behavior of the tanks with equal water $\begin{array}{ll}\text { depths } & 77\end{array}$

5.10 Maximum sloshing heights from ACI 350-3-06 and nonlinear FE $\begin{array}{ll}\text { analysis } & 84\end{array}$

5.11 Maximum sloshing heights from ACI 350-3-06 and nonlinear FE $\begin{array}{ll}\text { analysis } & 84\end{array}$

5.12 Effect of bidirectional seismic excitation on maximum sloshing height 85

$5.13 \quad$ Normalized sloshing heights 86

5.14 Orthogonality effect estimated by ASCE 7-05 and nonlinear FE analysis 87

5.15 Peak FE sloshing heights under Northridge, El-Centro, and San$\begin{array}{ll}\text { Francisco (unidirectional excitation) } & 88\end{array}$

5.16 Maximum time history sloshing heights for El-Centro and Northridge 89

5.17 Relative maximum sloshing heights 90

5.18 Maximum hydrodynamic pressure in model "SX40Z20" 96

5.19 Maximum hydrodynamic pressure in model "SX30Z15" 98

5.20 Maximum hydrodynamic pressure in model "SX20Z10" 100

5.21 Maximum hydrodynamic pressure in model "SX16Z8" 101 


\section{List of Figures}

2.1 Schematic single spring mechanical model (adapted from ACI 350.306(2006))

2.2 Convective and impulsive pressures distribution along the height of the tanks wall (adapted from ACI 350.3-06)

2.3 fluid motion in tank (adapted from ACI 350.3-06)

3.1 Coordinate system used for the derivation of sloshing equations (adapted from Ghaemmaghami (2010))

3.2 Schematic of equivalent mechanical model for lateral sloshing (adapted from Gaemmaghami (2010))

5.1 Nonlinear sloshing by Coupled Eulerian Lagrangian (CEL) analysis

5.2 Schematic view of a rectangular liquid tank (adapted from Ghaemmaghammi (2010))

5.3 Liquid domain mesh: (a) conventional mesh, (b) pseudo particles $\quad 60$

5.4 Schematic view of tank configurations (adapted from T.Avval (2012)) 62

5.5 Scaled horizontal components of the 1940 El-Centro record; (a) N-S, (b) E-W

5.6 Water sloshing in tank model "SX30Z15" 63

5.7 Time history of sloshing height for model "SX50Z25" 65

5.8 Time history of sloshing height for model "SX40Z20" 65

5.9 Time history of sloshing height for model "SX30Z15" 66

5.10 Time history of sloshing height for model "TX20Z10" 66

5.11 Time history of sloshing height for model "TX16Z8" 67

5.12 Time history of sloshing height for model "SX50Z25" 69

5.13 Time history of sloshing height for model "SX40Z20" 70

5.14 Time history of sloshing height for model "SX30Z15" 70

5.15 Time history of sloshing height for model "TX20Z10" 70

5.16 Liquid sloshing contour for TX20Y40: (a) whole model (b) sectional view through the tank 
5.17 Time history of sloshing height for model "TX16Z8" 78

5.18 Time history of sloshing height for model "TX20Z10" 79

5.19 Time history of sloshing height for model "SX30Z15" 79

5.20 Time history of sloshing height for model "SX40Z20" 80

5.21 Time history of sloshing height for model "SX50Z25" 80

5.22 Time history of sloshing height for model "TX16Z30" 81

5.23 Time history of sloshing height for model "TX20Z40" 82

5.24 Time history of sloshing height for model "SX30Z60" 82

5.25 Time history of sloshing height for model "SX40Z80" 82

5.26 Time history of sloshing height for model "SX50Z100" 83

5.27 Time history of sloshing height for model "SX40Z20" under El-Centro 91

5.28 Time history of sloshing height for model "SX40Z20" under Northridge 91

5.29 Time history of sloshing height for model "SX30Z15" under El-Centro 92

5.30 Time history of sloshing height for model "SX30Z15" under Northridge 92

5.31 Time history of sloshing height for model "TX20Z10” under El-Centro 93

5.32 Time history of sloshing height for model "TX20Z10" under Northridge 93

5.33 Time history of sloshing height for model "TX40Z15" under El-Centro 94

5.34 Time history of sloshing height for model "TX40Z15" under Northridge 94

5.35 Maximum hydrodynamic pressure in model "SX40Z20" 97

5.36 Maximum hydrodynamic pressure in model "SX30Z15" 99

5.37 Maximum hydrodynamic pressure in model "TX20Z10" 101

5.38 Maximum hydrodynamic pressure in model "SX16Z8" 102 


\section{Chapter 1}

\section{Introduction}

\subsection{General overview}

The dynamic behavior of fluid materials interacting with moving structures has attracted the attention of engineers in many fields in the last few decades. The fluid structure interaction (FSI) effects must be considered in design of numerous types of industrial plants such as dams, liquid storage tanks, spacecraft, suspension bridges, off-shore structures, ships, and nuclear plants. Because of the different nature of motions in those structures mentioned above, the dynamic behavior of the retained liquid changes from transient to cyclic types; hence, the dynamic FSI has become the subject of many studies in need to introduce precise models which can foresee the performance of liquid-filled tanks under dynamic loadings.

The liquid storage tanks are one of the essential structures which are required nowadays in everyday life, especially in emergency times. The liquid storage tanks provide safe and clean source of drinking water, and they are used for storing oils, petroleum materials, and other kinds of chemical materials. Hence, it is clear that their maintenance and continuous function is crucial for urban areas. Their significance becomes more critical after natural disasters such as major earthquakes. A safe source of water is needed for extinguishing the common fire hazards after severe seismic events in the past. In recent years, there has been a great deal of concern over possible primary or long-term catastrophic safety issues relating to public facilities, structures, and industrial plants. Therefore, those structures should be designed in order to remain safe during natural disasters.

Based on the most of practical design codes and standards such as ACI 360.3-06, liquid tanks are either ground-supported or pedestal-mounted structures. Ground supported tanks can be classified according to the following features:

- General configuration (rectangular or circular)

- Wall based joint type (fixed, hinged, or flexible base)

- Method of construction (prestressed or reinforced concrete) 
In previous studies, heavy damages to liquid storage tanks during strong earthquakes have been. Therefore, the poor performance of liquid tanks under seismic excitations has become clear. Damaged liquid storages and collapsed tanks have provided a great deal of motivation for engineers to develop more precise mechanical models and simulations to provide better estimations of the seismic response of the tanks during earthquakes. Many research and studies attempted to discover fundamental factors which can have significant impact on the fluid structure interaction properties.

At present, most of the codes and standards such as ACI 350.3 (2006) has adapted to one of the most commonly used analytical calculation developed by Housner (1957), which divides the total dynamics load into the impulsive and convective pressures. The impulsive pressure is associated with the movement of rigid container wall, and the convective part is in accordance with the vertical oscillation of the retained liquid. The tank wall is assumed to be rigid; nevertheless, many studies show that the wall flexibility can increase the impulsive portion of the pressure acting on the wall. It should be noted that the flexibility of the wall has negligible effect on the convective pressure.

Studies conducted on the dynamic response of liquid storage tanks revealed that a broad set of fundamental elements affect the behavior of these systems; in fact, the soil structure interaction effects (SSI) as well as the fluid structure interaction effects (FSI) should be considered in the dynamic analysis of liquid contained structures on their contact surfaces. To some extent, fluid properties and the features of the soil located under the liquid tank play important role on such analysis. Moreover, other factors including tank configurations, contained liquid properties, the depth of stored liquid, and the flexibility of structures should be deeply investigated in order to design efficient structures against dynamic loadings. Finally, when a tank partially filled with the liquid is prone to external acceleration, motion of the liquid free surface can cause dynamic loads either on the side walls or roof of the tanks. This load is called sloshing-induced load, and it depends on the frequency content of liquid oscillation, the peak ground acceleration and velocity of motions, the duration of loadings and the quantity of ground acceleration.

ACI 350.3-06 calculates the dynamic pressure acting on the wall and liquid sloshing height. The code calculation is based on a single component of seismic motion along the longer 
side of the liquid tank plan. It should be noted that an earthquake loading has 3 components along $\mathrm{x}, \mathrm{y}$, and $\mathrm{z}$ directions in reality. Many studies have been conducted on the impact of bidirectional horizontal earthquake loads and also some of them investigated the effect of vertical component of seismic motions. For example, Ghaemmaghami and Kianoush (2010) have conducted an intensive study to investigate the effect of vertical ground acceleration. They conducted a linear elastic analysis on 2D and 3D tanks considering all components of seismic ground motions. Ghaemmaghami (2010) studied the fluid structure interaction effects in flexible rectangular concrete tanks excited with four different earthquake records. Also Panchal et al. (2008) conducted a study on the seismic response of steel liquid storage tanks excited by six different ground movement records.

In well-known codes and standards mostly used around the world, linear mass-spring mechanical models are used to calculate the seismic design loads, stresses and vertical displacements of the liquid free surface (sloshing height). In these types of analytical methods the nonlinear characteristics of liquids, tanks, and seismic loadings are ignored. This assumption is unrealistic, because the nonlinear impacts are always presented and in some cases it may prevail over the linear calculated responses. To satisfy this requirement some theoretical and experimental studies have been conducted and some analytical solutions are available to determine the nonlinear sloshing response. For example, Goudarzi, and Sabbagh-Yazdi (2012) have conducted a thorough investigation on the nonlinear sloshing problem in seismically excited tanks. In addition, Juan C. Virella et al. (2008) studied the two-dimensional nonlinear and linear analysis of sloshing modes and pressures in rectangular tanks subjected to harmonic excitations.

There is a major concern over the large amplitude sloshing motions which occurs when waves with longer periods (5 to 10 or more seconds) are produced. The impact pressure acting on the tank roof could be significantly high and the associated damage on the roof will be considerable. The pressure acting on the roof is an important matter which should be the subject of studies in future.

The nonlinearity can affect every aspect of the performance of seismically excited liquid storage tanks. The resulted pressures and sloshing motions considering the nonlinearity are the 
major concerns of this study, and it is hoped that this study leads to a better understanding of the behavior of liquid storage tanks under seismic loadings.

\subsection{Methods of Analysis}

There are three distinct methods for calculating the dynamic response of liquid tanks under seismic loadings. Each type of those analyses can be employed to reach certain goals and has its advantages and disadvantages. The simplified method, the response-spectrum modal analysis, and the time-history analysis are widely used for predicting the dynamic response of liquid tanks subjected to earthquake ground motions.

First, the simplified method is used for preliminary calculation of stresses and forces. the traditional seismic coefficients are employed for computing the seismic design forces in rigid and nearly rigid tanks. In this type of calculation added mass of fluid due to the oscillation of the fluid inside the tank, and the inertia forces made by the side walls movements are represented by equivalent static forces applied at equivalent center of gravity of the moving system. To incorporate the effect of time dependant parameters each design code provides its appropriate coefficients. The inertia forces simply can be calculated from the product of structure mass or the added mass of the liquid. Provided that the stored liquid in the system is incompressible, the equivalent added mass of the liquid can be used for representing the fluid structure interaction analysis. It is important to note that this type of analysis is valid when the fluid motion are at the frequency much greater than that of the fundamental frequency of the tank motion. These types of preliminary solutions are derived by Westergaard (1938), Housner (1957), and Chopra (1975). Generally, simplified methods are foundations for later possible more refined procedures when proposed.

Second, the response-spectrum modal analysis computes the maximum linear elastic response of concrete tanks under seismic loadings. Maximum values of sloshing height, stresses and forces produced in systems can be obtained with simplified formulation assuming the liquidfilled structure remains fully linear elastic during dynamic loadings. This procedure calculates the maximum amount of stresses, forces, and displacements separately for each mode of the 
ground excitation. Then the critical modes, and multi-component earthquake inputs will be combined.

The square Root of Sum of Squared (SRSS) can be used for combing separate modal responses. This type of calculation can make an adequate estimation when the modes of excitation are well separated. On the other hand, Complete Quadratic Combination (CQC) can be employed when the vibration modes are closely spaced. In addition, the responses from each component of seismic excitations can be combined using either SRSS or QCQ type for multicomponent ground shakings. Response-spectrum modal analysis contains certain important limitations which are worth to be considered for evaluation of its results. The time dependant features of each seismic motion are significant parameters which have an impact on the dynamic response of hydro systems. Those effects are not addressed in this method. Moreover, all of calculated forces, stresses, and displacements based on this method are positive, and nonconcurrent; thus, the deformation plot of the system can't be estimated. Finally, the fluid structure interaction effects and the foundation structure interaction effects can be introduced approximately.

Finally, the time-history analysis is introduced to avoid the limitations mentioned above for the simplified method and response-spectrum modal method. The input data of seismic ground motions in this type of calculation is in the form of ground acceleration records in sequential time increment steps, hence, many major characteristics of a ground motion such as the loading time periods, number of excitation cycles, and existence of high energy pulses can be considered in this time dependant method of calculation. Moreover, the fluid-structure, and foundation-structure effects can be better addressed in the dynamic response of those structures.

\subsection{Objective and Scope of Study}

The current study is mainly focused on the nonlinear time-history analysis of liquid storage tanks. Based on numerous studies have been conducted on the dynamic response of liquid tanks, there are many factors that must be considered in design of liquid storage concrete tanks. 
The nonlinearity of liquid motions can perform distinctive effects on the performance of moving liquid tanks under dynamic loadings. In addition, there are other aspects that should be investigated in order to make a better understanding of the performance of liquid tanks under seismic loadings. These include three-dimensional geometry effects, sorted water levels, plan dimension aspect ratios, different earthquake components, long duration seismic loads, the frequency content of ground motions, and liquid sloshing waves. The main aim of this study is to investigate some of these significant parameters to foresee the behavior of liquid storage tanks subjected to seismic ground motions considering the nonlinearity of fluid motions and it is aimed to improve the design criteria of liquid storage tanks under seismic loadings. The major subjects whom this thesis will consider are as follows:

1. Proposing a nonlinear finite element analysis of concrete rectangular tanks that can be accurately employed in studying the nonlinear sloshing phenomenon and the fluid structure interaction effects with ABAQUS/CAE finite element method software package.

2. Studying the value of nonlinear sloshing heights at critical locations of rectangular concrete tanks.

3. Investigating the nonlinear liquid sloshing motions considering the wave interference effect using different horizontal components of earthquake records.

4. Studying the nonlinear total pressure acting on the tank wall.

5. Investigating the effect of frequency content of ground motions on the sloshing height values using three different seismic records.

6. Conducting a parametric study on the effect of different water levels and its aspect ratio with length of the tank side walls.

7. Developing a parametric to investigate the effect of the tank plan dimensions on the values of liquid sloshing height at the different location of rectangular tanks.

Based on studies conducted on dynamic fluid structure interaction problems, the wall flexibility considerably increases the pressure acting on tank walls due to the deformation occurs through seismic ground motions. On the other hand, the wall flexibility has no major effect on the sloshing height values and the convective part of the pressures, so the flexibility of the wall is 
ignored in this study. Moreover, dynamic behavior of the tanks with cracked wall section has negligible consequences on convective pressures and sloshing displacements of the liquid.

The vertical component of earthquake motions has shown to have no important effect on the value of convective pressures; therefore, the vertical component of earthquake records is ignored.

\subsection{Thesis Layout}

This thesis contains six chapters. In the first chapter, the importance of liquid storage structure is discussed and general information about liquid retained tanks is provided. Different available methods which are capable of analysing the fluid structure interaction phenomenon are briefly discussed. Finally, the scope and the objectives of this study are indicated.

The second chapter contains materilas which represent the importance of understanding the performance of seismically excited liquid storage tanks; in addition, the common types of damages to liquid storage tanks both for steel and concrete tanks are highlighted. The ACI 350.306 method of calculation of pressures, stresses, forces, and maximum liquid sloshing heights is described in Chapter two. Major investigations and academic research studies which have studied the fluid structure interaction problem are provided in this chapter.

Mathematical backgrounds including basic equations governing all marine structures subjected to earthquake loadings are presented in third chapter. These formulations are adapted for rectangular liquid storage tanks under seismic ground motions. The Housner's (1963) mechanical model for estimating the dynamic fluid structure interaction is discussed in Chapter three.

Different types of dynamic fluid structure interaction simulations used in computer programs are briefly discussed in Chapter four. The Smoothed-Particle-Hydrodynamic (SPH) analysis is discussed in this chapter. Finally, the method of finite element method conversion to SPH simulation is described. The SPH method is used in this study to simulate the liquid storage tanks under seismic loadings using ABAQUS/CAE (ver.2016) FEM software package. 
The application of the FEM calculation converted to the SPH simulation of the liquid storage tanks in ABAQUS/CAE is discussed in the fifth chapter. The nonlinear response of liquid storage tanks subjected to single unidirectional and bidirectional component (Longitudinal and transversal) of El-Centro (1960) seismic record is calculated. The effect of different tank plan dimension is discussed. The effect of wave interference on the sloshing performance of liquid-filled tanks at different locations is investigated in chapter five. The nonlinear pressures and sloshing heights are compared with the linear results. Calculated Results based on ACI 350.3-06 code are compared with the nonlinear dynamic response to investigate the accuracy of code in design of rectangular concrete tanks. Finally, the total nonlinear pressure acting on the tank wall is discussed in this chapter.

In chapter six, a summary of the results will be provided, in addition the major conclusions and possible recommendations for the design procedure of rectangular liquid storage tanks are suggested in this chapter. 


\section{Chapter 2}

\section{Literature Review}

\subsection{Introduction}

In this chapter, prior research and other related studies conducted on fluid-structure interaction problems in liquid-filled tanks under dynamic loading are discussed. Section 2.2 includes the dynamic behavior of liquid tanks under seismic loading and historical damages mentioned in past studies (i.e. earthquake events around the world). Moreover, the most common failure mechanisms of different liquid tank types will be briefly described. Section 2.3 reviews the important contribution of those prior studies that address the performance of seismically excited liquid storage tanks. Finally, the design code and others related studies will be discussed.

\subsection{Seismic Damage to Liquid Storage Tanks}

Several previous reports have demonstrated different types of damages to liquid storage tanks caused by strong earthquakes. Different failure mechanisms of liquid storage tanks during previous seismic events exhibit that certain characteristics of liquid storage tanks including material properties, supporting systems and tank configurations are significant factors that make a certain type of a container more- or lees- vulnerable to a specific kind of damage.

The strong 1964 Alaska earthquake was the subject of several studies. Reports revealed that oil storage tanks, most of which were unanchored at the base received considerably more damage than other types of fluid containers. The shell buckling phenomenon was observed as a common type of failure in most of the unanchored oil storage tanks. Moreover, the uplift that occurred around the exterior edge of the tank bottom plate is reported in most of those studies. Hanson (1973) implied that seismic forces could cause uplift at the bottom of the tank wall at the edge.

During the 1979 Imperial Valley earthquake, the buckling of the shell due to excessive axial compressive stresses, and the failure of fixed roofs because of the liquid sloshing effect, 
were the types of damages reported by Haroun (1983). The failure mechanisms observed due to the earthquake of the 1983 Coalinga event were mostly seen on oil cylindrical tanks. Manos and Clough (1982) observed bottom plate and joint rupture, liquid spilling over, elephant-foot buckling, top shell buckling, and roof-pipe connection corruption as the most common damages which oil storage tanks experienced in that strong seismic ground motion.

In the 1964 Niigata and 1999 Kocaeli earthquakes, the observed damages were roof failure and the flow of liquid over the top of the tank wall due to the excessive amount of liquid sloshing height. Liquid tanks at the time of those destructive earthquakes were designed based on the design code of California (1961) and they had metallic seals. Due to the surface sloshing of oil in tanks, the metal to metal connections between walls and metallic seals caused sparks that ignited the stored fuel inside the tanks. Massive long-time firing hazards took place after both earthquakes in highly industrial locations and caused a vast amount of oil loss. The 1964 Niigata, 1964 Alaska and 1999 Kocaeli earthquakes revealed the poor performance of oil storage tanks during strong earthquakes. The possible failure of these types of liquid storage tanks would be huge economic and public hazards in urban areas, so further studies and investigations on this subject are essential.

In the 1994 Northridge earthquake, many critical lifeline plants were severely damaged in Los Angeles. In the 1971 San Fernando earthquake, most water storage tanks experienced severe damage. Buckling was the most common type of damage. In addition, several tanks were damaged because of excessive liquid sloshing (Lund (1996)).

Steel tanks experienced severe damage based on available reports during previous earthquake events. Failure mechanisms that are related to steel tanks commonly include one or more of the following categories:

- The buckling of the shell, which is more common in cylindrical steel tanks and; it takes place when a high overturning moment causes high axial compression in the walls of liquid tanks.

- The roof damages occur because of the excessive liquid sloshing effect in tanks with insufficient freeboard or excessive convective pressure acting on the upper part of the wall of tanks. 
- The anchorage failure which occurs in liquid containers with restrained bases.

Concrete tanks also received severe damage during past earthquakes. It should be noted that concrete tanks are designed for full serviceability performance; thus, disruption of tank functionality or leakage more than specified limits allowed in codes and standards are considered as failures of this type of liquid container. Damages to concrete tanks due to the seismic ground motion can be classified as follows:

- Supporting system failure.

- Leakage in the reservoir and adjoining wall connection, and vertical cracks in expansion joints.

In addition to all damages and failure mechanisms mentioned above, there are other types of damages, based on information gained from previous earthquake events, which both concrete and steel tanks could receive. These types of damages are as follows:

- The liquid spilling over the top of the tank's wall because of the surface liquid sloshing effect.

- The foundation failure.

- Differential settlements at the base of the tanks.

- The failure of accessories which are connected to the tanks because of the excessive movement during earthquakes.

- The interior roof's column failure.

Based on the previous observations, liquid tanks can be subjected to a tremendous amount of hydrodynamic pressure. In concrete tanks, the additional stress could be generated by inertia due to the mass of the concrete, which could lead to leakage or even collapse of the structure.

The mentioned damages to liquid storage tanks in past strong earthquakes have attracted the attention of engineers in many fields, engineers who have conducted many research studies on the dynamic performance of liquid-filled tanks. Those investigations have made for a better understanding of the complex behavior of such structures under seismic loadings. 


\subsection{Previous Research Studies}

The investigations of fluid-structure interaction problems began in the 1930's when Westergaard (1933) investigated the pressure acting on the wall of a rectangular dam subjected to horizontal earthquake excitations. The first investigation of the seismic performance of liquid tanks was conducted by Jacobsen (1949) who studied the same problem on cylindrical, rectangular, semicircular, triangular and hemisphere liquid tank configurations under horizontal seismic motions. The first experimental study was done by Werner and Sundquist (1949) as an extension of Jacobsen's (1949) work.

The first practical solution for calculating hydrodynamic pressure was proposed by Housner $(1957,1963)$ for both cylindrical and rectangular tanks. This procedure was carried out to find solutions to satisfy Laplace's equation for specific boundary conditions of liquid volumes. He divided the stored liquid into two parts. First, the impulsive part which moves rigidly with the tank wall due to the inertia forces produced by the motion of side walls. The impulsive pressure is acting on the wall at the lower part of side walls. Second, the convective part is proportional to vertical oscillation of the liquid free surface. He proposed a simplified method of calculation of hydrodynamic pressures using a lumped mass approach under two horizontal components of earthquake motion. The tank is rigid at the base. The impulsive mass is rigidly connected to the tank wall while the convective mass is attached to the wall by an equivalent spring as shown in Figure 2.1. The liquid is assumed to be incompressible and inviscid, and tank walls are supposed to behave rigidly. However, later studies have shown that flexibility of the tank's walls has a significant effect on the amount of impulsive pressure acting on the wall. This model with some modifications has been used in the most practical codes and standards. It should be noted that this model ignores the effect of higher sloshing modes of vibrations.

Epstein (1976) based on Housner's model fundamentals, produced design curves which can be used to approximately estimate the overturning and bending moments, which are the result of hydrodynamic pressures acting on the liquid tank's wall.

Haroun (1983) conducted an experimental study on three tanks with real dimensions under different vibrations including ambient and forced types to determine the mode shapes and natural frequencies of those motions. Then, Haroun (1984) proposed a refined analytical solution 
for calculating the total impulsive pressure using the classical potential flow theory for incompressible, inviscid, and homogenous fluids. The wall of the tank is assumed to be rigid. Haroun's solution calculates lower pressure at the lower part of the wall while it estimates the higher pressure at the upper elevation of the wall in comparison with the results calculated by the Housner model. He calculated shear lateral forces and stresses for two different base supporting systems. The tank walls and the base are rigidly supported in the first system while the tank's side walls are free to move in the second one. Finally, he evaluated bending moment coefficients, which most of the codes and standards have been using.

Marchaj (1979) studied the impact of the vertical component of earthquake movement on the hydrodynamic pressure induced in liquid storage tanks subjected to dynamic loading. He assumed horizontal stripes to study the behavior of liquid excited by the vertical component of different ground motions. He suggested that the vertical component of the earthquake could be an important variable affecting the performance of moving liquid containers. Haroun and Tayel (1985) used a finite element method incorporating the effect of the vertical seismic component for analyzing the dynamic response of liquid tanks. Their method is based on the superposition of asymmetrical vibrational modes on the cylindrical tank in which the tank wall behaves elastically. While the sloshing effect was ignored, axial and radial deformation of the wall and corresponding stresses were calculated. Moreover, Veletsos and Kumar (1984) investigated the effect of the vertical component of earthquake motion in tanks with a flexible base. They found that the flexibility of the soil beneath the tank may decrease the amount of hydrodynamic pressure.

Veletsos and Yang (1976) conducted an extensive study of the dynamic performance of vibrating liquid storage tanks involving the effect of wall flexibility. They developed a simple approximate approach to predict the effect of flexibility on the hydrodynamic pressure induced on the side walls. Finally, they studied natural frequencies and modes of vibration of both empty tanks and filled-tanks by liquid. Veletsos et al. (1992) proposed a detailed solution to calculate the impulsive and convective components separately. They found out that the flexibility of the wall has a tremendous impact on the impulsive portion of the total hydrodynamic pressure.

Park et al. (1992) studied the dynamic behavior of rectangular tanks using the coupled boundary element method (BEM) and finite element method (FEM). They used FEM to 
investigate the solid wall and BEM to calculate the hydrodynamic pressure distribution. They used the time domain analysis technique to find a solution capable of calculating both convective and impulsive components of hydrodynamic pressures.

Subhash and Bhattacharyya (1996) used FEM to produce a numerical model for computing the sloshing height, and to estimate the associated pressure acting on the wall of liquid tanks. Koh et al. (1998) by using the boundary element method for the liquid domain in combination with the finite element method presented a numerical calculation for analyzing three-dimensional seismically excited rectangular tanks.

Choun and Yun (1999) investigated the sloshing response of rectangular tanks using the linear analytical approach. They considered only the horizontal component of ground movement. They discovered that the properties of ground movement and the tank configuration have a significant impact on the sloshing response of the rectangular tank. They suggested that a lowfrequency seismic ground motion increases the amount of sloshing height.

Chen and Kianoush (2005) used the sequential method to calculate The hydrodynamic pressure considering the wall flexibility effect for two-dimensional (2D) tanks. Later, Kianoush and Chen (2006) investigated the dynamic performance of the tank considering the vertical component of ground motion, and also they presented a new method of calculation of dynamic behavior of 2D rectangular tanks, which calculates both convective and impulsive parts of dynamic pressure using Time-History analysis.

Akyildiz and Unal (2005) developed experimental studies to investigate the nonlinear behavior and damping features of the fluid sloshing response in rectangular baffled and unbaffled liquid storage tanks. Also, they investigated the hydrodynamic pressure distribution at different locations of the rectangular tank. They concluded that the baffles considerably decrease the amount of resulting liquid sloshing height. Akyildiz and Unal (2006) in another similar study observed that the severity of the sloshing behavior of the liquid depends on parameters such as tank configuration, amplitude and nature of the ground motion, and frequency content of the ground vibration.

Virella et al. (2008) conducted a study on both linear and nonlinear sloshing responses of two-dimensional rectangular concrete tanks; also, he calculated natural periods and mode shapes 
of the fluid motion using both linear and nonlinear wave theories. He suggested that the resulting nonlinear pressure induced by the sloshing response was not significantly different from the linear one.

Pal and Bhattacharyya (2010) conducted numerical and experimental studies on the liquid sloshing performance of two-dimensional partially liquid-filled tanks under dynamic loadings. They used the meshless local Petrov-Galerkin method to calculate the nonlinear response of prismatic liquid tanks. They also investigated the sloshing behavior of prismatic liquid tanks experimentally. They implied that the nonlinear effect of the sloshing response could be critical when the amplitude of vibration becomes large and the frequency of the motion is near to the natural frequency of the retained liquid volume.

Goudarzi and Sabbagh-Yazdi (2012) investigated the nonlinear sloshing effects in a partially liquid-filled tank subjected to seismic excitations. They simulated both nonlinear and linear conditions. They verified linear simulation with the previous analytical solutions while they used experimental setups to validate their proposed nonlinear solution. They implied that under a nonlinear sloshing effect the positive sloshing heights grow faster than the negative ones, and maximum upward sloshing heights are larger than the downward sloshing heights at the liquid free surface. They implied that the effect of nonlinearity on liquid sloshing is more apparent in wider tanks.

Kianoush and Ghaemmaghami (2011) conducted intensive studies on rectangular tanks considering both fluid-structure interaction (FSI) and soil-structure interaction (SSI). They introduced a finite element technique which estimates convective and impulsive pressure components considering wall flexibility on seismically excited rectangular liquid storages. Moreover, they investigated the frequency content of ground motion by modeling tanks with different dimensions under four different seismic records. Finally, they studied sloshing height values at different locations of rectangular tanks. They concluded that medium frequency content ground movements would make the largest maximum sloshing heights in the corners of rectangular liquid tanks.

Under the light of previous research and studies, it is clear that many different parameters should be investigated further to provide a better understanding of the performance of 
seismically excited liquid-filled tanks. These parameters include tank configurations, components of a ground motion, the nonlinear effects of the liquid sloshing and the frequency content of earthquake motions.

\subsection{Design Codes and Standards}

The effect of dynamic fluid-structure interaction should be considered for designing of a broad variety of structures which contained or are surrounded by liquid when they are subjected to earthquake loadings. Although the fluid-structure interaction problems are different in each type of those marine or hydro structures, hydrodynamic analysis of all of them uses similar basic equations. The objective of this section is to describe the process of designing liquid storage tanks subjected to earthquake loadings based on ACI 350.3 (2006).

Many codes and standards are available to design liquid storage tanks such as ACI 350.306 (2006). ACI 350.3 is one of the most comprehensive and practical standards. Unlike some of the other standards that are for designing steel tanks or contain the procedure for circular tanks, ACI0350.3 covers the design criteria of all types of concrete liquid storage tanks. The committee

in charge of creating the code implied that liquid storage tanks should be recognized as structures not covered adequately by other national codes and standards. This code provides a full earthquake analysis and design of fluid storage concrete tanks.

The ACI 350.3 committee implies that the code includes some significant developments in comparison with prior methodologies. Those are as follows:

- In rigid tanks, the acceleration of tank motion is equal to ground acceleration motion. This code considers the wall's flexibility, assuming amplification of response due to the natural frequency of the tank.

- Effect of vertical acceleration of ground motion is considered in this standard.

- The combination of convective and impulsive parts of the hydrodynamic response should be calculated by the square-root-sun-of-squares (SRSS) formula.

- An effective mass coefficient applicable to the mass of the wall is included in this code. 
- This standard contains the response modification factor.

In this section, the calculation of the hydrodynamic response of rectangular concrete tanks based on ACI 350.3-06 will be represented.

In order to design the side walls of liquid-filled rectangular tanks in addition to the static pressures, the following dynamic forces must be considered. Those dynamic induced loads are as follows:

- Inertia forces of the wall $\left(\mathrm{P}_{\mathrm{w}}\right)$

- Liquid hydrodynamic forces including impulsive pressure $\left(\mathrm{P}_{\mathrm{i}}\right)$ and convective pressure $\left(\mathrm{P}_{\mathrm{c}}\right)$

- Dynamic earth pressure caused by saturated and unsaturated types of soil against the buried part of the wall.

- The impact of the vertical acceleration component of dynamic loads.

The general equation for calculating the total base shear of the structure $\left(V=C_{S} W\right)$ is modified by incorporating four effective weights: $\varepsilon W_{W}$ (effective weight of wall), $\left(W_{r}\right)$ (effective weight of roof), $W_{i}$ (weight of impulsive part of liquid), and $W_{c}$ (weight of convective part of liquid) instead of $W$. Moreover, $C_{S}$ should be replaced by $C_{i}, C_{c}$, and $C_{v}$, as appropriate. In this section, a more detailed description will be provided about the convective and impulsive parts. It is important to note that convective and impulsive properties of a liquid are not in the same phase; therefore, ACI 350.06-3 uses the square-root-sum-of-the-squares (SRSS) method for combining these components to calculate the total response. The dynamic lateral forces above the ground shall be calculated as follows;

Dynamic Inertia Force of the Wall:

$$
P_{W}=C_{i} I\left[\varepsilon W_{W} / R_{i}\right]
$$


Impulsive Force:

$$
P_{i}=C_{i} I\left[W_{i} / R_{i}\right]
$$

Convective Force:

$$
P_{c}=C_{c} I\left[W_{c} / R_{i}\right]
$$

The code uses a mechanical equivalent model. In this model, the impulsive mass is rigidly connected to the tank's walls, and the convective mass is attached to the walls by equivalent springs. Figure 2.1 illustrates the schematic model used in ACI 350.3-06.

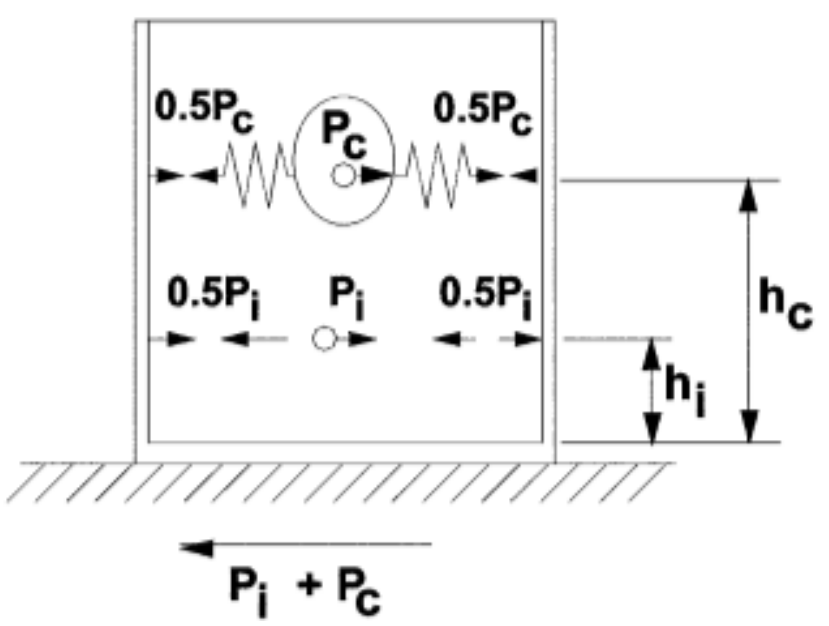

Figure 2.1: Schematic single spring mechanical model (adopted From ACI 350.3-06(2006))

As mentioned before, the hydrodynamic forces generated during a seismic event acting on the wall are in distinct phases; therefore, to combine their effect on the wall, the SRSS method should be used to calculate the total dynamic response acting on the wall as follows:

$$
V=\sqrt{\left(P_{i}+P_{w}\right)^{2}+P_{c}^{2}}
$$


Where, $I$ is the importance factor which should be selected according to the expected use of the tank. Moreover, $\left(R_{i}\right)$ is the response modification factor, and it should be chosen based on the system that the tank is connected to the ground at the base and the elevation of the tank base in comparison with the ground level. These two parameters can be chosen according to Table2.1 and Table2.2, respectively:

Table 2.1: Response modification factor $R_{i}$, and $R_{c}$ (adapted From ACI 350.3-06(2006))

\begin{tabular}{|c|c|c|c|}
\hline \multirow{2}{*}{ Type of Structure } & \multicolumn{2}{|c|}{$R_{i}$} & \multirow{2}{*}{$R_{c}$} \\
\cline { 2 - 3 } & On or above ground & Buried & \\
\hline Anchored or flexible-base tanks & 3.25 & 3.25 & 1 \\
\hline Fixed or hinged-base tanks & 2 & 3 & 1 \\
\hline Unanchored, contained or uncontained tanks & 1 & 2 & 1 \\
\hline Pedestal-mounted tanks & 2 & 0 & 1 \\
\hline
\end{tabular}

Table 2.2: Importance factor I (adapted From ACI 350.3-06(2006))

\begin{tabular}{|c|c|}
\hline Tank Use & $I$ \\
\hline Tank containing hazardous materials & 1.5 \\
\hline $\begin{array}{c}\text { Tanks that are intended to remain usable for emergency } \\
\text { purposes after an earthquake or tanks are part of lifeline } \\
\text { structures }\end{array}$ & 1.25 \\
\hline Tanks not listed in category 1 or 2 & 1 \\
\hline
\end{tabular}

It should be noted that buried tanks are defined by the elevation of liquid free surface at rest; if the liquid free surface is below the ground level, tanks should be defined as buried tanks.

The code allows engineers to consider the value of the Importance Factor more than 1.5 provided that the tank is filled with a hazardous contaminated fluid.

In this section, the calculation of dynamic forces acting on rectangular concrete tanks externally excited by seismic loads will be described. The ACI 350.3-06 procedure is based on Housner's model that uses a multi-mass system connected to the tank wall representing the equivalent convective weight effect $\left(W_{c}\right)$ of liquid and also the equivalent impulsive weight effect $\left(W_{i}\right)$. Convective mass is connected with the spring while the impulsive mass is attached 
rigidly to the wall of the tank in this model. Each of these masses is connected to the wall at equivalent heights.

Impulsive Mass:

$$
W_{i}=W_{L} \times \frac{\tanh \left[0.866\left(\frac{L}{H_{L}}\right)\right]}{0.866\left(\frac{L}{H_{L}}\right)}
$$

Convective Mass:

$$
W_{c}=W_{L} \times 0.264\left(\frac{L}{H_{L}}\right) \tanh \left[3.16\left(\frac{H_{L}}{L}\right)\right]
$$

Center of gravity for equivalent impulsive mass:

For tanks with $\frac{L}{H_{L}} \leq 1.33$

$$
h_{i}=H_{L} \times\left[0.5-0.09375\left(\frac{L}{H_{L}}\right)\right]
$$

For tanks with $\frac{L}{H_{L}}>1.33$

$$
h_{i}=H_{L} \times 0.375
$$

It should be noted that equations of $\left(h_{i}\right)$ which are mentioned above are based on an idea that the base pressure is excluded from the impulsive pressure acting on the wall.

Center of gravity for equivalent convective mass:

$$
h_{c}=H_{L} \times\left(1-\frac{\cosh \left[3.16\left(\frac{H_{L}}{L}\right)\right]-1}{3.16\left(\frac{H_{L}}{L}\right) \sinh \left[3.16\left(\frac{H_{L}}{L}\right)\right]}\right)
$$

$C_{c}$ and $C_{i}$ are seismic coefficients. Prior to calculating these seismic coefficients, the natural frequencies of the impulsive and convective parts of the liquid should be estimated.

Circular Impulsive frequency: 


$$
\omega_{i}=\sqrt{\frac{k}{m}}
$$

Structural stiffness $(\mathrm{k})$, shall be computed based on the appropriate boundary condition. For tanks fixed at the base, the tank's wall is assumed to behave like a free-top cantilever; therefore, flexural stiffness for a unit width of the wall $(\mathrm{k})$ could be estimated by the following equation in SI system:

$$
k=\frac{E_{c}}{4 \times 10^{6}}\left(\frac{t_{w}}{h}\right)^{2}
$$

The mass parameter in equation (2.10) includes impulsive mass component $\left(m_{i}\right)$ which simply could be computed by dividing $\mathrm{W}_{\mathrm{i}}$ by the gravity acceleration value. The $\mathrm{m}_{\mathrm{w}}$ could be computed as follows:

$$
m=m_{i}+m_{w}
$$

Where, $\mathrm{m}_{\mathrm{w}}$ is;

$$
m_{w}=H_{W} \frac{t_{w}}{12}\left(\frac{\gamma_{c}}{g}\right)
$$

Convective circular frequency:

$$
\omega_{c}=\frac{\lambda}{\sqrt{L}}
$$

Where, $\lambda$ is defined by:

$$
\lambda=\sqrt{3.16 g \tanh \left[3.16\left(\frac{H_{L}}{L}\right)\right]}
$$

Finally, the convective natural frequency can be calculated using the following equation:

$$
T_{C}=\frac{2 \pi}{\omega_{C}}=\left(\frac{2 \pi}{\lambda}\right) \sqrt{L}
$$

Impulsive seismic coefficients $\left(C_{i}, C_{c}\right)$ can be estimated by the following equations: Where, $T_{i} \leq T_{s}$ 


$$
C_{i}=S_{D S}
$$

And if $T_{i}>T_{S}$

$$
C_{i}=\frac{S_{D 1}}{T_{i}} \leq S_{D S}
$$

Where, $T_{s}$ is equal to:

$$
T_{S}=\frac{S_{D 1}}{S_{D S}}
$$

And for $T_{c} \leq \frac{1.6}{T_{S}}$

$$
C_{c}=\frac{1.5 S_{D 1}}{T_{c}} \leq 1.5 S_{D S}
$$

Unless,

$$
C_{c}=\frac{2.4 S_{D S}}{T_{c}^{2}}
$$

It should be noted that $\mathrm{S}_{\mathrm{D} 1}$ is the spectral response acceleration at the one second period; in addition, $\mathrm{S}_{\mathrm{DS}}$ is the short period spectral response spectral acceleration. These factors can be computed by equations (2.22) and (2.23). As mentioned at the beginning of this section, $\mathrm{S}_{\mathrm{S}}$ and $S_{1}$ are mapped maximum response spectral accelerations for at the short period and at the one second period, respectively. They should be extracted in accordance with the earthquake ground movement map (ASCE 7-05, Chapter 22). $F_{a}$ and $F_{v}$ are coefficients related to site characteristics and should be found in accordance to the site classification table provided by ASCE 7-05.

$$
\begin{aligned}
& S_{D S}=\frac{2}{3} S_{S} F_{a} \\
& S_{D 1}=\frac{2}{3} S_{1} F_{v}
\end{aligned}
$$

Impulsive and convective parts of hydrodynamic response at any given height of the rectangular tank's wall above the base can be computed by the following equations: 


$$
\begin{aligned}
P_{i y}= & \frac{\frac{P_{i}}{2}\left[4 H_{L}-6 h_{i}-\left(6 H_{L}-12 h_{i}\right)\left(\frac{y}{H_{L}}\right)\right]}{H_{L}^{2}} \\
P_{c y}= & \frac{\frac{P_{c}}{2}\left[4 H_{L}-6 h_{c}-\left[6 H_{L}-12 h_{c}\right]\left(\frac{y}{H_{L}}\right)\right]}{H_{L}^{2}}
\end{aligned}
$$

Figure 2.3 illustrates the pressure distribution proportional to the height of the wall which is perpendicular to the seismic ground motion direction.
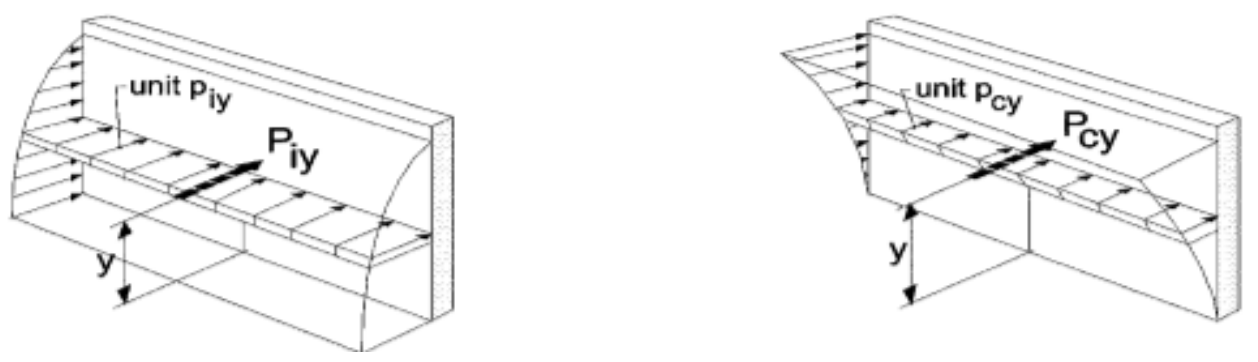

Figure 2.2: Convective and impulsive pressure distribution along the height of the tank's wall (ACI 350.3-06 (2006))

Based on ACI 350.3-06 (2006) the wall-to-floor, wall-to-wall, and wall-to-roof joints of rectangular tanks should be designed to resist seismic shear forces on the walls which are perpendicular or parallel to ground motion directions. For estimating the shear forces in the first group, the wall should be analyzed as slabs under horizontal pressures. The shear forces along the bottom, side and roof-to-wall (if applicable) joints should be responded by slab reactions. Moreover, the latter group shall be analyzed as shear walls subjected to the in-plane forces. Based on these considerations, bending moment, and overturning moment can be computed.

The bending moment induced in the liquid tank externally excited by the dynamic load consists of impulsive, convective and bending moment components that are caused by the wall inertia. Bending moment $M_{b}$ can be determined according to the following equations: 


$$
\begin{gathered}
M_{W}=P_{W} \times h_{W} \\
M_{i}=P_{i} \times h_{i} \\
M_{c}=P_{c} \times h_{c} \\
M_{b}=\sqrt{\left(M_{i}+M_{W}\right)^{2}+M_{c}^{2}}
\end{gathered}
$$

Liquid storage tanks should be designed to maintain the required freeboard height to avoid spilling the contained liquid over the edges and also to resist the impact pressure acting on the tank's roof.

Provided that the site-specific response is employed for a 5\% damping ratio of critical damping, the maximum vertical displacement of the liquid free surface shall be computed by the following equation based on the code as follows:

$$
d_{\max }=\left(\frac{L}{2}\right)\left(C_{c} I\right)
$$

On the other hand, if the site-specific response spectrum is for a damping ratio other than $5 \%$ of critical damping, the effect of damping on the spectral amplification has to be considered for calculating the maximum sloshing height. $\eta_{c}$ resembles the effect of damping on the spectral amplification.

$$
d_{\max }=\left(\frac{L}{2}\right) I\left(\eta_{c}\right) \frac{\left(0.667 S_{D}\right)}{g}\left(\frac{2 \pi}{T_{c}}\right)^{2}
$$

Where,

$$
\eta_{c}=\frac{3.043}{2.73-0.45 \ln \beta}
$$

Figure 2.3 illustrates how the standard predicts the sloshing motion of the liquid free surface in liquid tanks subjected to the seismic loadings. 


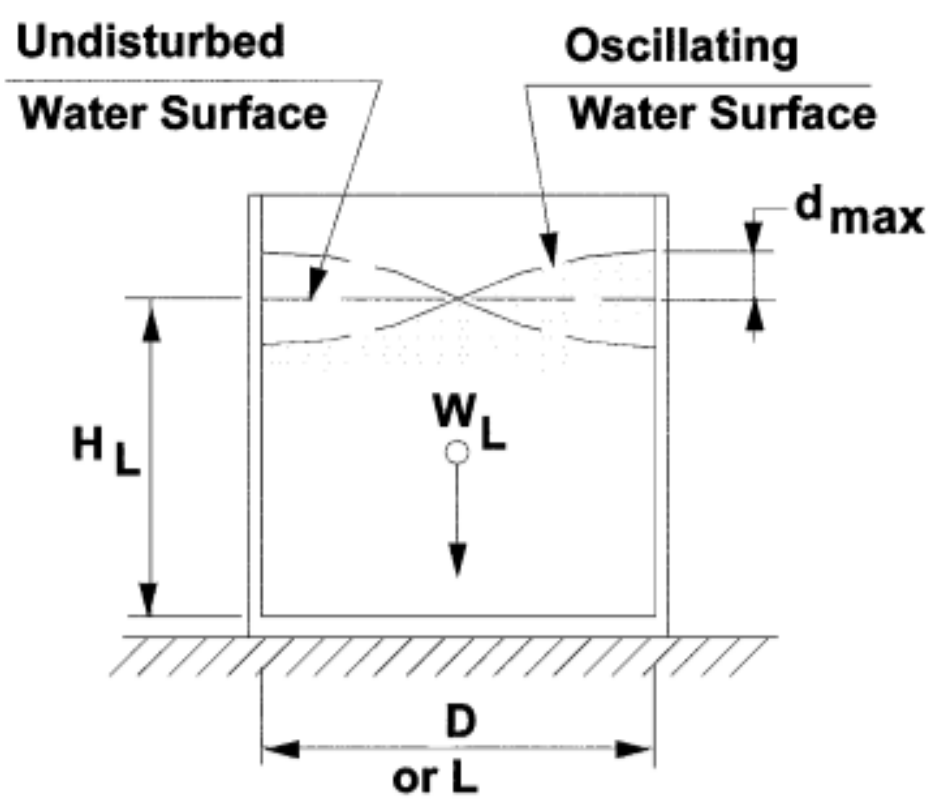

Figure 2.3: Fluid motion in tank (ACI 350.3-06 (2006)) 


\section{Chapter 3}

\section{Mathematical Background}

\subsection{Introduction}

In this chapter, an analytical solution to the dynamic response of liquid storage tanks will be discussed. The maximum sloshing wave height (MSWH) and hydrodynamic pressures are the major issues which should be considered in the dynamic analysis of rectangular liquid-filled tanks. A more accurate analytical solution which estimates hydrodynamic pressures acting on the tank wall by liquid motion, and vertical displacement of the free liquid surface during seismic excitations is needed in order to make an accurate picture of such dynamic responses and also to compare with the more refined solutions that may be proposed in the future.

As mentioned in previous chapters, the dynamic performance of liquid storage tanks is a complicated phenomenon, and it is sensitive to many distinct parameters. The classical potential theory is capable of calculating the hydrodynamic forces and sloshing wave heights in liquid storage tank systems externally excited by dynamic loadings. During earthquake events, liquid oscillation utilizes gravity scalar waves at the liquid free surface. The forces acting on the tank wall by the liquid motion under horizontal components of the seismic movement can be divided into impulsive and convective portions. It should be noted that in this theory the liquid is assumed to be inviscid, incompressible, and homogenous; in addition, the tank's walls are assumed to behave rigidly. The basic formulation of fluid-structure interaction in rigid tanks with those assumptions will be described in this section. Also the general mechanical model that is mostly used in current codes and standards will be briefly discussed in this chapter.

Most equations that are presented in this section for predicting the hydrodynamic forces and sloshing heights in rigid liquid storage tanks are adapted from NASA SP-106 procedure which was introduced to deal with fluid-structure interaction in moving liquid-filled tanks. The equations represented in this chapter are revised in a way so as to be applicable to the rectangular storage tanks. It should be noted that most of the material presented in this section is adapted from Ghaemmaghami's (2010), Ph.D. thesis for the Civil Engineering Department of Ryerson 
University. Equations represented in this section are extracted from the mentioned reference for providing a more detailed literature review on the complex dynamic liquid sloshing problems.

\subsection{Theories for Dynamic Analysis of Liquid Storage Tanks}

Two different theories are available for analyzing the dynamic performance of fluid storage tanks. Housner $(1957 ; 1963)$ using lamina fluid theory introduced a model for the vibrational analysis of liquid storage tanks. In this model, the tank is assumed to behave rigidly, and the liquid volume is assumed as an equivalent lumped mass having two degrees of freedom. The effect of higher order sloshing modes is ignored in this method, so it is not capable of analyzing the coupled vibrational systems.

The theory of velocity potential is the other method that can be used to analyse the dynamic behavior of liquid-filled tanks. The liquid volume is regarded as potential flow in this theory. Using specific boundary conditions along the contact surface between fluid particles and the wall of the container, the motion of the liquid contained in the tank can be described by this theory.

\subsection{Velocity Potential Theory Governing Equations}

In this section, to discuss the dynamic performance of rectangular tanks under seismic excitation, the Cartesian coordinate system $(\mathrm{x}, \mathrm{y}, \mathrm{z})$ is employed. All of the differential equations and boundary conditions will be represented using the Cartesian coordinate system in this section. Figure 3.1 illustrates a rectangular tank with a plan dimension of $2 \mathrm{a}$ by $2 \mathrm{~b}$ in $x$ and $y$ directions, respectively. 


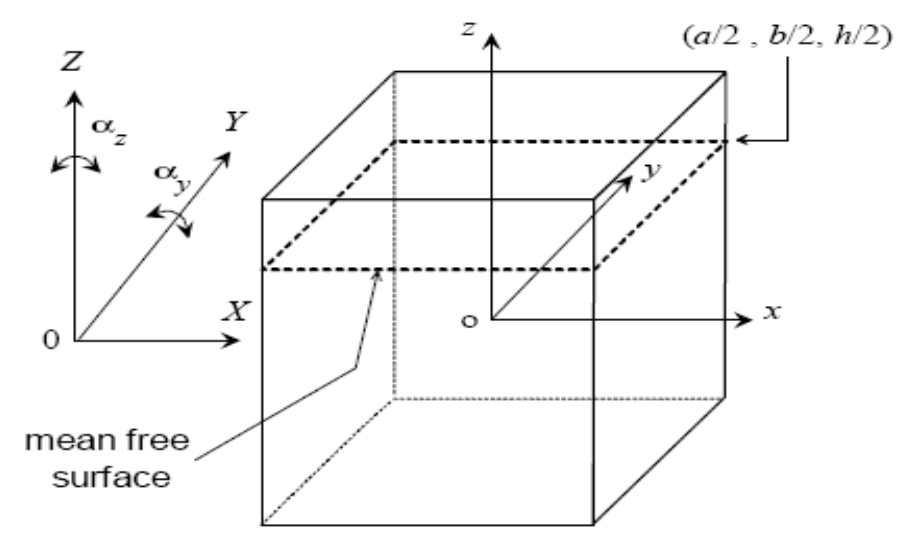

Figure 3.1: Coordinate system used for the derivation of sloshing equations (Ghaemmaghami (2010))

In fluid dynamics, potential flow describes the velocity field in a gradient of a scalar function. The potential function $\phi(x, y, z, t)$ can be defined as velocity potential where the liquid is assumed to be incompressible and inviscid. Moreover, the function can satisfy the La Palace's equation when the flow of the liquid is considered as an irrotational flow. By these definitions, the components of velocity in Cartesian coordinates can be defined as follows:

For incompressible fluids, the following expression can be implied;

$$
\nabla \times \nabla \phi=0
$$

By definition of irrotational flow;

$$
\nabla \times \vec{V}=0
$$

Thus;

$$
\vec{V}=\nabla \phi
$$

So velocity components $\mathrm{u}, \mathrm{v}$, and $\mathrm{w}$ along $\mathrm{x}, \mathrm{y}$, and $\mathrm{z}$ axes in Cartesian system, can be obtained from the derivation of velocity potential function $(\phi)$ as follows:

$$
u=\frac{\partial \phi}{\partial x} \quad v=\frac{\partial \phi}{\partial y} \quad \text { and } w=\frac{\partial \phi}{\partial z}
$$


As the fluid is assumed to be incompressible, the basic differential equation should satisfy the La Place's equation as well as the conservation of mass equation at any points in liquid domain:

$$
\begin{gathered}
\text { La Place Equation } \Rightarrow \nabla^{2}=0 \\
\frac{\partial^{2} \phi}{\partial x^{2}}+\frac{\partial^{2} \phi}{\partial y^{2}}+\frac{\partial^{2} \phi}{\partial z^{2}}=0 \\
\frac{\partial u}{\partial x}+\frac{\partial v}{\partial y}+\frac{\partial w}{\partial z}=0
\end{gathered}
$$

One of the most widely used equations in fluid mechanics is the Bernoulli function. The Bernoulli function assumes that the liquid flow is frictionless and ignores the effect of heat transfer or work. For a potential flow with no vorticity, the dynamic equation of motion can be integrated directly to obtain the unsteady Bernoulli's equation:

$$
\rho\left\{\frac{\partial}{\partial t} \nabla \phi+\frac{1}{2}(\nabla \phi)^{2}\right\}+\Delta \rho+\rho g \nabla z=0
$$

With the substitution of potential function into the Bernoulli equation, equation (3-8) can be rewritten as follows:

$$
\begin{gathered}
\nabla\left\{\rho \frac{\partial \emptyset}{\partial t}+\frac{1}{2} \rho V^{2}+P+\rho g z\right\}=0 \\
\frac{\partial \phi}{\partial t}+\frac{P}{\rho}+g z+\frac{1}{2}\left(u^{2}+v^{2}+w^{2}\right)=f(t)
\end{gathered}
$$

Where, $P$ is the fluid pressure, $\rho$ is the density of the liquid, $g$ is the gravity acceleration in the negative direction of z-axes and $f(t)$ is the constant which is the product of the integration.

Velocities $u, v$, and $w$ are assumed to be small, so the square value of them are too small to consider; therefore, their effects can be ignored in linear terms. As long as the constant of integration is a function of time, it can be added to the definition of $\phi$ whenever it is convenient. As a result, the linearized form of the unsteady Bernoulli's equation can be written in the following form: 


$$
\frac{\partial \phi}{\partial t}+\frac{P}{\rho}+g z=0
$$

The possible solutions for equation (3.7) must satisfy certain boundary conditions. For example, for boundaries such as a free liquid surface or contact surface between the tank and the liquid, the La Palace equation should be satisfied. The free surface of liquid contains two boundary conditions. First, there are no restrictions at the surface of the liquid, and the liquid is free to move, so the fluid pressure at the surface is zero. As a result, the Bernoulli equation for particles located at the surface can be written as follows:

$$
\frac{\partial \phi(x, y, z, t)}{\partial t}+g \delta(x, y, t)=0 \text { for } z=\frac{h}{2}
$$

$\delta(x, y, t)$ is the small vertical surface motion above the undisturbed level of the fluid. Another specific boundary condition at the free surface is related to the kinematic properties of the liquid motion. The liquid surface is free to move, so the derivation of $\delta(x, y, t)$ with respect to the time would provide the vertical component of velocity of the liquid:

$$
\frac{\delta(x, y, t)}{\partial t}=w=\frac{\partial \phi}{\partial z}
$$

From the combination of equations (3.12) and (3.13), equation (3.12) can be rewritten completely in terms of $\phi$ as follows:

$$
\frac{\partial^{2} \phi}{\partial t^{2}}+g \frac{\partial \phi}{\partial t}=0 \text { for } z=\frac{h}{2}
$$

The derivative of the $\phi$ function with respect to time would lead to the natural frequency of sloshing. The main conclusion from equation (3.14) is that the natural frequency of the vertical displacement of liquid at the free surface is directly proportional to the gravitational scalar.

The only boundary condition that can be used from the contact surface of the wall and the liquid is associated with the velocity of liquid particles. By assuming that the contained liquid is inviscid, the liquid velocity component perpendicular to the wall should be equal to the wall's velocity component perpendicular to its plane at the wetted interface. 
Since the sloshing problem discussed in this section is linear, the whole problem can be separated into individual problems. Each type of the tank excitation of interest can be solved separately, and ultimately results can be added together to reach the velocity potential for a complete excitation.

The motion of the tank is assumed to be harmonic, and also it is a function of time; therefore, the function of this movement can be assumed to be as $\exp (i \Omega t)$, where $\Omega$ is the frequency of the tank excitation. Consequently, the displacement and the velocities can be expressed as follows:

$$
\begin{gathered}
X(t)=-i X_{0} \exp (i \Omega t) \\
v=w=0 \\
u=i X_{0} \Omega e^{i \Omega t}
\end{gathered}
$$

Where, $\mathrm{n}$ stands for a unit value vector perpendicular to the wall plane. The other conclusion that can be drawn from the wetted surface of the wall is that $u=\frac{\partial \phi}{\partial x}$, so we can rewrite equation (3.17) as follows:

$$
\text { n. } \nabla \phi=i X_{0} \Omega e^{i \Omega t}=\frac{\partial \phi}{\partial x}
$$

\subsection{Solution of Equation for a Rectangular Tank}

In this section, for the tank which has been shown in Figure 3.1, possible solutions of the excitation are the subject of the discussion. It should be noted that this method is adapted from NASA-SP-106. The tank is assumed to be rigid at the base. As discussed before, the potential interest is assumed to be harmonic in time such as $\exp (i \omega t)$. In this discussion the effect of time on $\phi$ function can be assumed mostly negligible; however, when time derivatives are required, they can be included by multiplying the potential function by $i \omega$. For this problem, the potential solutions are called the Eigen functions of the problem.

Eigen functions are founded by a method of separation of variables which is adapted from NASA-SP-106. Eigen functions are assumed to be the result of three distinct functions 
$\xi(x), \psi(y)$ and $\zeta(z)$ expressed in Cartesian coordinates. By combining these functions with equation (3.7), the following equations can be obtained:

$$
\begin{gathered}
\phi=\xi \psi \zeta \\
\frac{1}{\xi} \frac{d^{2} \xi}{d x^{2}}+\frac{1}{\psi} \frac{d^{2} \psi}{d y^{2}}+\frac{1}{\zeta} \frac{d^{2} \zeta}{d z^{2}}=0
\end{gathered}
$$

The expressions in equation (3.20) should satisfy at the boundary conditions located at the wetted surface of the wall as well as at the free surface of the liquid. Solutions of equation (3.20) will readily develop the natural frequency of the sloshing for the liquid tank systems. The equation of the natural frequency of sloshing will be as follows:

$$
\omega=\sqrt{\pi(2 n-1)\left(\frac{g}{a}\right) \tanh \left[\pi(2 n-1)\left(\frac{h}{a}\right)\right]}
$$

where $\mathrm{n}$ stands for the number of the vibrational modes. From equation (3.21), it can be concluded that by increasing the depth of liquid, the natural frequency of sloshing would become longer; however, by increasing the length of the wall along the direction of the dynamic load the natural frequency would be decreased. Finally, the equation shows that the lowest natural frequency occurs in the first mode $(n=1)$ of vibration.

Provided that the tank is excited in $x$-direction, equation (3.17) for the bottom of the tank can be rewritten as follows:

$$
\begin{gathered}
u=\frac{\partial \phi}{\partial x}=\Omega X_{0} e^{i \omega t} \text { for } x= \pm \frac{a}{2} \\
v=\frac{\partial \phi}{\partial y}=0 \text { for } y= \pm \frac{b}{2} \\
w=\frac{\partial \phi}{\partial z} \text { for } z= \pm \frac{h}{2}
\end{gathered}
$$

The boundary condition at the bottom of the tank is the same as the boundary condition at the free surface; hence, the trial solution for this case of excitation will be determined as follows: 


$$
\phi(x, z, t)=\left\{A_{0} x+\left\{\cosh \left[\lambda_{n} \frac{z}{a}\right] \tanh \left[\lambda_{n} \frac{h}{2 a}\right] \sinh \left[\lambda_{n} \frac{z}{a}\right]\right\}\right\}
$$

In the previous equation, $\mathrm{n}$ indicates the number of the modes. The expression $(2 n-1) \pi$ is replaced by $\lambda_{n}$ and $A_{n}$ is the constant of integration.

At the bottom of the tank (where, $z=\frac{h}{2}$ ), $v$ and $w$ are zero; therefore, $\phi$ potential satisfies the boundary condition only if $A_{n}$ becomes equal to $\Omega X$.

In the next step, equation (3.25) should be satisfied at the boundary condition at the liquid free surface. In order to do that, $\phi$ should be inserted into the equation (3.25), which gives:

$$
-\Omega^{2}\left\{\Omega X_{0} x+\sum_{i=1}^{\infty} A_{n} \sin \left(\lambda_{n} \frac{x}{a}\right)\left[\cosh \left(\lambda_{n} \frac{h}{2 a}\right)+\tanh \right]\right\}=0
$$

Equation (3.26) indicates that the constant of integration $\left(A_{n}\right)$ can be expressed in terms of $X_{n}$, to make an approximate determination, the $x$ in the first term should be written as the function of $\sin \left(\frac{\lambda_{n}}{a}\right)$, which is possible because the $\sin \left(\frac{\lambda_{n}}{a}\right)$ terms are orthogonal within $-\frac{a}{2} \leq x \leq \frac{a}{2}$. This process gives:

$$
x=\sum_{i=1}^{\infty}\left(\frac{4 a}{\lambda_{n}^{2}}\right)(-1)^{n-1} \sin \left(\frac{\lambda_{n} x}{a}\right)
$$

By substituting $\mathrm{x}$ from equation (3.27) into the equation (3.25), the final function of velocity potential will become available as follows:

$$
\begin{aligned}
& \phi(x, z, t) \\
& =A_{0} e^{i \Omega t}\left\{x \sum_{i=0}^{\infty} \frac{4 a(-1)^{n-1}}{\lambda_{n}^{2}}\left(\frac{\Omega^{2}}{\omega_{n}^{2}-\Omega^{2}}\right) \sin \left[\lambda_{n} \frac{x}{a}\right] \frac{\cosh \left(\lambda_{n}\left[\frac{z}{a}+\frac{h}{2 a}\right]\right)}{\cosh \left[\lambda_{n} \frac{h}{a}\right]}\right\}
\end{aligned}
$$

It is important to remember that the forces acting on the tank's wall, practically in design practices, are separated into convective and impulsive components. The first one is the product of the oscillation of the liquid in the tank at the free surface level, while the impulsive forces are 
generated because of the movement of the wall, so it's clear that sloshing and impulsive forces are in independent phases. The impulsive potential function can be obtained as follows:

$$
\begin{aligned}
& \phi_{I}(x, z, t) \\
& =A_{0} e^{i \Omega t}\left\{\sum_{i=1}^{\infty} \frac{4 a(-1)^{n-1}}{\lambda_{n}^{2}} \sin \left[\lambda_{n} \frac{x}{a}\right] \frac{\cosh \left[\lambda_{n}\left(\frac{z}{a}+\frac{h}{2 a}\right)\right]}{\cosh \left[\lambda_{n} \frac{h}{a}\right]}\right\}
\end{aligned}
$$

By assuming that $\phi=\phi_{C}+\phi_{I}$, the convective part of potential function should be easily obtained as follows:

$$
\begin{aligned}
& \phi_{c}(x, z, t) \\
& =A_{0} e^{i \Omega t}\left\{\sum_{i=1}^{\infty} \frac{4 a(-1)^{n-1}}{\lambda_{n}^{2}}\left(\frac{\Omega^{2}}{\omega_{n}^{2}-\Omega^{2}}\right) \sin \left[\lambda_{n} \frac{x}{a}\right] \frac{\cosh \left[\lambda_{n}\left(\frac{z}{a}+\frac{h}{2 a}\right)\right]}{\cosh \left[\lambda_{n} \frac{h}{a}\right]}\right\}
\end{aligned}
$$

In order to determine the forces acting on the tank's wall caused by liquid sloshing, the unsteady part of the velocity potential function should be integrated. Providing that $d F_{x}=\rho d A_{x}$ is the acting force in $x$-direction, and perpendicular to the wall's plane on the element with the area of $d A_{x}$, the forces acting on the wall in $x$-direction can be computed as follows:

$$
F_{x}=2 \int_{-h / 2}^{h / s+\delta} \int_{-b / 2}^{b / 2} p d y d z=-2 \rho b \int_{-h / 2}^{h / 2} \frac{\partial \phi}{\partial t} d z
$$

By substituting $\phi$ into equation (3.28) and also by linearizing equation (3.31) with respect to the unsteady pressure and wave amplitude on the wall the integration would give:

$$
\frac{F_{X 0}}{-i \Omega^{2} X_{0} m_{l}}=1+8 \frac{a}{h} \sum_{n=1}^{N} \frac{\tanh \left[\lambda_{n}\left(\frac{h}{a}\right)\right]}{\lambda_{n}^{3}} \frac{\Omega^{2}}{\omega_{n}^{2}-\Omega^{2}}
$$

Where, $F_{X 0}$ is the force, and $m_{l}=\rho a b h$ is the total mass of liquid retained in the tank.

Finally, the moment exerted on the tank base about y-axes can be calculated. The differential moment is $M_{y}=-x\left(p d A_{x}\right)-z\left(p d A_{x}\right)$; hence, the total moment can be calculated by integration of the differential moment as follows: 


$$
\begin{gathered}
M_{y}=2\left(\int_{-h / 2}^{h / 2+\delta} \int_{-b / 2}^{b / 2} z p d y d_{z}-\int_{0}^{a / 2} \int_{-b / 2}^{b / 2} x p d_{y} d_{x}\right) \\
M_{y}=2 b \int_{-h / 2}^{h / 2} z \frac{\partial \phi}{\partial t} d z-2 b \int_{0}^{a / 2} x \frac{\partial \phi}{\partial t} d x
\end{gathered}
$$

After solving the integration, applying a series of simplification by algebraic transformations and rearranging the results, the following equation is the ultimate solution to calculate the moment:

$$
\begin{aligned}
\frac{M_{y 0}}{-i \Omega^{2} X_{0} m_{l} h}= & \frac{1}{12}\left(\frac{a}{h}\right)^{2} 8\left(\frac{a}{h}\right) \sum_{n=1}^{\infty} \frac{\tanh \left[\lambda_{n}(h / a)\right]}{\lambda_{n}^{3}} \\
& \times\left\{\frac{1}{2}-\frac{2(a / h) \tanh \left[\lambda_{n}(h / 2 a)\right]}{\lambda_{n}}+\frac{g}{h \omega_{n}^{2}}\right\} \frac{\Omega^{2}}{\omega^{2}-\Omega^{2}}
\end{aligned}
$$

By ignoring the effect of the free surface motion, both the force acting on the wall, and the moment acting on the base of the tank can be computed by the following equations:

$$
\begin{aligned}
& \frac{F_{x o}}{-i \Omega^{2} X_{0} m_{l} h}=1-8 \frac{a}{h} \sum_{n=1}^{N} \frac{\tanh \left[\lambda_{n}(h / a)\right]}{\lambda_{n}^{3}} \\
& \frac{M_{y o}}{-i \Omega^{2} X_{0} m_{l} h}=\frac{1}{2}-8 \frac{a}{h} \sum_{n=1}^{\infty} \frac{\tanh \left[\lambda_{n}(h / a)\right]}{\lambda_{n}^{3}}
\end{aligned}
$$

\subsection{Analytical Derivation of Mechanical Model Parameters}

The influence of the sloshing waves on the dynamic performance of liquid storage tanks under seismic loading was discussed in the previous section. An equivalent mechanical model which represents the actual dynamic situation could be more convenient to use than the analytical equations of the liquid motion described in the last section. Such a mechanical model must include significant features in order to become capable of solving complex dynamic problems including the Fluid-Structure interaction. In most of the linear mechanical models used for solving the dynamic FSI problem, the mass of liquid is replaced with equivalent masses 
connected to the tank wall with different types of connections. The number of the masses connected to a wall in the mechanical model system is equal to the number of vibrational modes in each specific dynamic loading. It should be noted that in most of these models a rigid body is assumed for the tank structure.

In this section, the Mass-Spring mechanical model will be described. In Figure 3.2, this mechanical model is shown. The dynamic forces acting on the liquid storage tank are separated into the impulsive and convective components. The impulsive mass of the liquid which is represented by $m_{0}$, is rigidly attached to the tank, and also any moment of inertia needed for the analysis is related to this mass. Convective masses are attached to the wall by springs, and the number of these masses required in the model is proportional to the number of dynamic vibration modes. The center of gravity is presented in the model is located in the liquid center of gravity. $H_{0}$ in the model stands for the height of the center of gravity of the equivalent impulsive mass, and each of $H_{1}, H_{2}, \ldots$, and $H_{n}$ represents the center of gravity of equivalent mass of the liquid mass for $n$th modes of the sloshing response. 


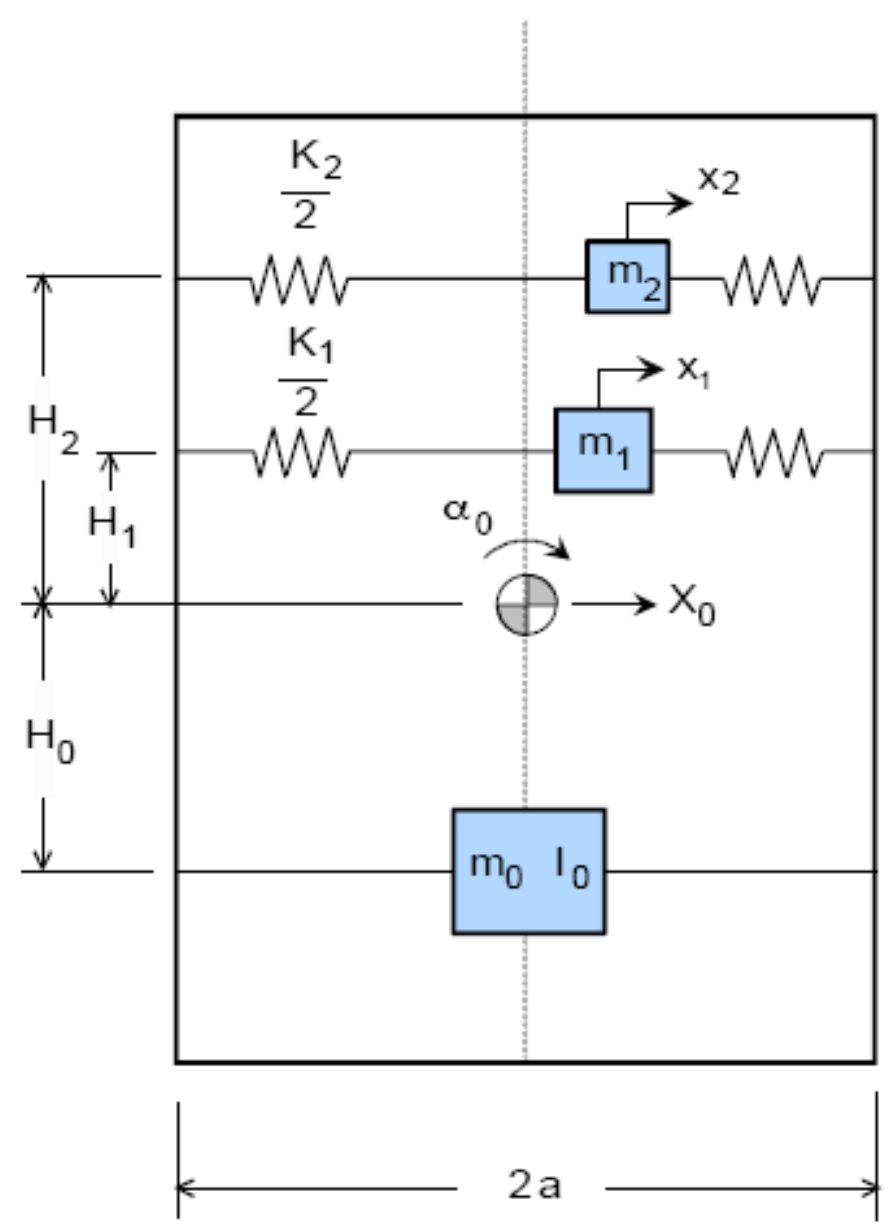

Figure 3.2: Schematic of equivalent mechanical model for lateral sloshing (Gaemmaghami (2010))

In order to maintain the static properties of the liquid retained in the tank, the sum of all equivalent masses in the model must be equal to the total mass of liquid retained inside the tank. The center of mass in the model should be at the same height as the center of gravity of the liquid volume is located. These constraints can be mathematically defined as follows:

$$
\begin{gathered}
m_{0}+\sum m_{n}=m_{l i q} \\
m_{0} H_{0}+\sum m_{n} H_{n}=0
\end{gathered}
$$


Moreover, the mechanical model should cover the calculation of forces, moments, and natural frequencies of the liquid sloshing motion in the tank. In order to compute the natural frequency of each mode of sloshing, the stiffness of the equivalent spring should satisfy the following equation:

$$
\omega_{n}=\sqrt{\frac{k_{n}}{m_{n}}}
$$

The calculation of moments and forces related to each equivalent mass can be used to develop other mathematical relations. In fact, the net force in the positive direction of $X$ is computed by the reverse inertia force of masses as follows:

$$
-F=m_{0} \ddot{X}_{0}+\sum m_{n}\left(\ddot{x}_{0}+\ddot{X}_{n}\right)
$$

In addition, the moment produced in the tank can be calculated by the following equation:

$$
-M=\sum m_{n} h_{n} \ddot{x}_{n}-g \sum m_{n} x_{n}
$$

The equation of the motion can be produced by considering the simple dynamic equation of the motion as below:

$$
\begin{gathered}
F=m a+k x=0 \\
m_{n}\left(\ddot{X}_{0}+\ddot{x}_{n}\right)+k_{n} x_{n}=0
\end{gathered}
$$

As was discussed in the previous section, providing that the tank excites by the frequency of $\Omega$, the displacement of the ground would be $X(t)=-i X_{0} \exp (i \Omega t)$; thus, equation (3.44) can be rewritten as follows:

$$
x_{n}=\frac{i \Omega^{2} X_{0}}{\omega_{n}^{2}-\Omega^{2}}
$$

Finally, by considering all of the mentioned equations, the equations of forces and moments can be expressed as: 


$$
\begin{gathered}
\frac{F_{a m p}}{-i \Omega^{2} X_{0} m_{\text {liq }}}=\left[1+\sum \frac{m_{n}}{m_{\text {liq }}}\left(\frac{\Omega^{2}}{\omega_{n}^{2}-\Omega^{2}}\right)\right] \\
\frac{M_{a m p}}{-i \Omega^{2} X_{0} m_{\text {liq }} h}=\sum \frac{m_{n}}{m_{\text {liq }}}\left(\frac{H_{n} \Omega^{2}+g}{\omega_{n}^{2}-\Omega^{2}}\right)
\end{gathered}
$$

For the calculation of force and moment amplitudes in equations (3.46) and (3.47), $m_{n}, \omega_{n}$ and $H_{n}$ should be chosen in a way that satisfies at certain boundary conditions. By comparing the last equations with the equation of the lateral force exerted on the tank's wall due to the sloshing motion, $m_{n}$ and $k_{n}$ can be expressed as follows:

$$
\begin{gathered}
m_{n}=\frac{8 m_{\text {liq }} a / h}{\pi^{3}(2 n-1)^{3}} \tanh \left[(2 n-1) \frac{\pi h}{a}\right] \\
k_{n}=\frac{8 m_{\text {liq }} g}{h \pi^{2}(2 n-1)^{2}}\left(\tanh \frac{\pi(2 n-1) h}{a}\right)^{2}
\end{gathered}
$$

Likewise, by comparing equation (3.48) to the equation of the moment produced in $\mathrm{x}$ translation of the model [equation (3.18)], the elevation of masses can be calculated by the following equation:

$$
\frac{H_{n}}{h}=\frac{1}{2}-2\left(\frac{a}{h}\right) \frac{\tanh \left(\frac{(2 n-1) h}{2 a}\right)}{2 n-1}
$$

Housner (1963) introduced a simplified Spring-mass model. Because the higher modes of sloshing can be negligible, his model contains a single mass connected to the wall by using springs. $M_{i}$ is the equivalent impulsive mass rigidly connected to the wall, and $M_{c}$ is considered as an equivalent convective mass connected by using springs to the wall. Because the tank is assumed to be rigid at the base, the acceleration of the impulsive part is equal to the acceleration of the ground motion.

The equivalent system for a rectangular tank is specified by the following equations:

$$
M_{i}=M \frac{\tanh 1.7(L / h)}{1.7 L / h}
$$




$$
\begin{gathered}
M_{c}=M \frac{(0.83) \tanh (1.6 \mathrm{~h} / L)}{1.6 \mathrm{~h} / L} \\
k_{c}=3 \frac{M_{c}^{2}}{M} \frac{g h}{L^{2}} \\
h_{i}=\frac{3}{8} h\left\{1+\alpha\left[\frac{M}{M_{i}}\left(\frac{L}{h}\right)^{2}-1\right]\right\} \\
h_{c}=h\left[1-\frac{M}{M_{c}}\left(\frac{L}{h}\right)^{2}-0.63 \frac{L}{h} \sqrt{0.28\left(\frac{M}{M_{c}} \frac{L}{h}\right)-1}\right]
\end{gathered}
$$

Amounts of $\alpha, \beta$ in equations are equal to 1.33 and 2.0 , respectively.

The maximum amount of liquid sloshing is proportional to the convective mass portion of the liquid domain. By assuming that the vertical displacement is equal to $A_{1} \sin \omega t$ at the free surface of the liquid beside the tank's wall interface, the liquid beside the tank wall would have a vertical displacement equal to $y=d \sin \omega t$; hence, the maximum sloshing height of the liquid in the rectangular tank can be computed as follows:

$$
d_{\max }=\frac{0.84 A_{c}\left(\frac{k_{c}}{M_{c}} \frac{g}{g}\right)}{1-\frac{A_{c}}{L}\left(\frac{k_{c 1}}{M_{c}} \frac{L}{g}\right)^{3}}
$$




\section{Chapter 4}

\section{Numerical Methods for Seismically Excited Liquid Storage Tanks}

\subsection{Introduction}

In the last few decades, there has been an enormous interest in fields such as computer science, engineering, and hydrodynamic studies that have introduced numerical simulations for analysis of the fluid-structure interaction. Numerical hydrodynamic methods are incredibly significant for designing liquid storage tanks.

To date, there are a number of numerical hydrodynamic simulations which have been adapted to the Smooth-Particle-Hydrodynamics (SPH) method of analysis which was introduced by Lucy (1977) and Gingold, and Monaghan (1977).

The method mentioned above has been the subject of modification designed to strengthen its abilities and solve its limitations. In this chapter, the different numerical simulation methods which are used nowadays in finite element computer software packages to solve hydrodynamic problems will be discussed. While a brief description will be presented about most of the known available methods, the Smooth-Particle-Hydrodynamic method, which is used in this study to simulate the liquid tanks used in this study with conversion to finite element method, will be discussed at length. It should be noted that the content presented in this chapter is mostly adapted from "A Smoothed Particle Hydrodynamics Method for the Simulation of Centralized Sloshing Experiments" written by Alexander Vorobyev (2012). The material presented in this section should be considered as a detailed literature review of that book. Equations provided in this book are represented in this section to provide a detailed literature review on SPH numerical analysis.

\subsection{Numerical Simulation Methods}

\subsubsection{Meshed-base Numerical Methods}

In the beginning, numerical solutions mostly used the Finite Difference Method (FDM) approach to analysis hydrodynamic problems. The liquid medium in this type of simulation is 
defined as an Eulerian element which makes possible the use of static mesh calculations. The liquid volume can flow across the entire volume of the Eulerian initially defined element while the mesh system defined at the beginning of the analysis remains fixed. The nodes of both liquid (Eulerian element), and the structure's wall (Solid element) are attached. The liquid is assumed to be in a system of parallel channels. FDM works well with "channel-type" geometries; nonetheless, in case of unusual loading conditions like seismic loading, the computational domain will drastically change because of the complex deformation in the liquid domain of the system.

In conclusion, for the situations which include fast and high deformations in liquid volume, the Eulerian approach shows a lack of accuracy, or even it is unable to run the analysis. The Eulerian methodology has several disadvantages:

- The Eulerian element should be defined to provide an imaginary geometry for the liquid to flow inside it; this element should overlap all elements to reflect correct boundary conditions.

- The Eulerian element creates difficulties in applying essential boundary conditions and constraints. In most of the programs the liquid element is defined as a volume fraction of Eulerian geometry; therefore, applying specific boundary conditions are impossible.

- The free surface displacement and geometry evolution during a dynamic loading are difficult to track down precisely.

\subsubsection{Lagrangian Method}

Another choice for simulating hydrodynamic problems is the Lagrangian method. In this method spatial coordinates are attached to the material; as a result, when the material's nodes move, spatial coordinates move simultaneously. The Lagrangian method has many useful characteristics in comparison with the Eulerian approach:

- Because of the attached computational nodes to the moving material, all major issues can be investigated by using the Time-History analysis.

- Any change of free surface geometry can be tracked down during the analysis period since computational nodes move with the moving material. 
- The transportation of physical parameters such as mass, velocity, acceleration, etc., are defined natively by the motion of nodes; hence, the convective term is excluded from governing equations( Vorobyev (2012))

The Lagrangian method overcomes some of most significant disadvantages of the Finite Different Method approach. One of the most well-known numerical methods introduced based on the Lagrangian method was the Finite Element Method (FEM) which is mostly used for the simulation of systems contained low relatively deformation in comparison with other systems containing fast and high deformation of liquid domain.

However, highly distorted mesh elements can be unavoidable during the fast deformation process of the liquid, and this may affect the accuracy of the numerical analysis, or the analysis may fail during the loading period. As is clear, remeshing distorted meshed elements is crucial to maintaining the acceptable accuracy of analysis, but reproducing new computational nodes based on the information from old nodes of distorted elements can also affect the validity of results.

\subsubsection{Smoothed Particle Hydrodynamics (SPH)}

Smooth particle hydrodynamic analysis is introduced in order to effectively simulate models involving extensive deformation such as liquid sloshing motions. This type of analysis considerably reduces the amount of computational time that is otherwise needed for a single nonlinear analysis.

For a problem such as liquid sloshing motion, a numerical method is required, one that is capable of simulating the following physical matters:

- Precise tracking down of liquid free surface motions.

- Accurate estimation of pressure waves which are produced by liquid motion acting on the contact surface between a structure's walls and the contained liquid.

- Formation of liquid particles, the interaction between particles representing the whole liquid domain together, and handling problems in which the physical material moves out of the computational domain.

The Smoothed Particle Hydrodynamics ( $\mathrm{SPH}$ ) is a complete mesh-free Lagrangian numerical method. Monaghan (1985) described this method as a kind of numerical approach for 
compressible flows. Nowadays, the SPH method has been developed extensively in order to simulate the fluid-structure interaction in liquid storage structures under seismic excitations. Different variants of the SPH approximations for enhancing accuracy, convergence, and stability are proposed. The SPH approach is widely used for the simulation of hydrodynamic problems. The ability to apply the SPH method to different hydrodynamic problems in comparison with the limitations of other well-known types, especially for the liquid free surface flow, made this method a desirable solution for the computer modeling of the liquid sloshing motion in this study. In this section, a brief explanation of SPH theory will be presented.

The smoothed particle hydrodynamic approach is based on an interpolation technique. The conserved volume of the fluid element discretizes to the points which resemble the specified material properties, which are called particles or pseudo-particles. Liu G.R. and Liu M.B. (2003) presented the approximation in two steps: the Kernel approximation and the discrete particle approximation.

The integral representation of the Kernel function can be expressed as follows:

$$
f(\vec{r})=\int_{\Omega}^{0} f\left(\overrightarrow{r^{\prime}}\right) \delta\left(\vec{r}-\overrightarrow{r^{\prime}}\right) d \overrightarrow{r^{\prime}}
$$

Where, $f(\vec{r})$ is continuous function. $\overrightarrow{r^{\prime}}$ is the radius vector. $\delta\left(\vec{r}-\overrightarrow{r^{\prime}}\right)$ is the Dirac delta function. The Dirac delta function can be defined as follows:

$$
\delta\left(\vec{r}-\overrightarrow{r^{\prime}}\right)=\left\{\begin{array}{l}
1, \vec{r}=\overrightarrow{r^{\prime}} \\
0, \vec{r} \neq \overrightarrow{r^{\prime}}
\end{array}\right.
$$

The Kernel function of $W\left(\vec{r}-\overrightarrow{r^{\prime}}, h\right)$ can be substituted into the Dirac delta function to smooth the liquid volume distribution and to determine the equation of liquid motion as follows:

$$
f(\vec{r}) \cong\langle f(\vec{r})\rangle=\int_{\Omega}^{0} f\left(\overrightarrow{r^{\prime}}\right) W\left(\vec{r}-\overrightarrow{r^{\prime}, h}\right) d \overrightarrow{r^{\prime}}
$$

In equation (3.3), $\mathrm{h}$ stands for the smoothing radius, and the angular bracket is the approximated value of the function $f$ at the location presented by the radius vector $\vec{r}$. 
In order to validate the satisfactory accuracy of the approximation, two special boundary conditions should be checked. First, the integration of the smoothing kernel over the domain of function should be equal to one. Moreover, when the smoothing radius approaches zero, the kernel function should become the Dirac delta function, which is called delta-function property. The expressions of both mentioned conditions are as follows, respectively:

$$
\begin{gathered}
\int_{\Omega}^{0} W\left(\vec{r}-\overrightarrow{r^{\prime}, h}\right) d \overrightarrow{r^{\prime}}=1 \\
W\left(\vec{r}-\overrightarrow{r^{\prime}, h}\right) \underset{h \mapsto 0}{\longrightarrow} \delta\left(\vec{r}-\overrightarrow{r^{\prime}}\right)
\end{gathered}
$$

In the second step, the continuous integral approximation should be discretized to a number of point nodes called, particles or pseudo-particles. The Lagrangian method is employed for capture the motion of particles. Each particle maintains the main characteristics of the liquid. The continuous field function $f(\vec{r})$ should be substituted by $f_{i}$ which represents the value of this function for different particles at various locations. Finally, for a discrete number of nodes, the integration in equation (3.5) can be substituted by a summation as follows:

$$
f_{i}(\vec{r})=\sum_{j} f_{j} W\left(\vec{r}_{l}-\overrightarrow{r_{j}{ }^{\prime}, h}\right) \Delta V_{j}
$$

Where, $\Delta V_{j}={ }^{m} / \rho_{j}$ is the volume of $j^{t h}$ particle. By replacing the volume expression into the latest formula, the SPH approximation of continuous function can be written as follows:

$$
f_{s}(\vec{r})=\sum_{j} f_{j} \frac{m_{j}}{\rho_{j}} W\left(\overrightarrow{r_{l}}-\overrightarrow{r_{j}, h}\right)
$$

$m_{j}$ and $\rho_{j}$ stand for mass and density $j^{\text {th }}$ particle, respectively. To sum up, the summation in the equation (4.7) governs the movement of particles in the liquid domain.

By use of the gradient of the smoothing kernel $\left(\nabla W\left(\overrightarrow{r_{l}}-\overrightarrow{r_{j}}, h\right)\right)$, the slope of the function can be readily obtained as follows:

$$
\nabla f_{i}(\vec{r})=\sum_{j} f_{j} \frac{m_{j}}{\rho_{j}} \nabla W\left(\overrightarrow{r_{l}}-\overrightarrow{r_{j}^{\prime}, h}\right)
$$

By derivation of the kernel, the slope of the kernel function can be obtained: 


$$
\nabla W\left(\overrightarrow{r_{l}}-\overrightarrow{r_{j}}, h\right)=\frac{\left(\vec{r}_{l}-\vec{r}_{J}\right)}{\left|\overrightarrow{r_{l}}-\vec{r}_{j}\right|} \frac{\partial W\left(\vec{r}_{l}, \vec{r}_{j}, h\right)}{\partial\left(\vec{r}_{l}-\vec{r}_{J}\right)}
$$

Where, $\frac{\partial W\left(\overrightarrow{r_{l}}, \overrightarrow{r_{J}}, h\right)}{\partial\left(\overrightarrow{r_{l}}-\overrightarrow{r_{J}}\right)}$ is the derivation of the smoothing kernel.

\subsection{Governing Equations of liquid motion in the SPH method.}

The main equations that govern the motion of incompressible, viscous, and homogenous liquid are as follows:

- The Navier-Stokes equations: These equations govern the conservation of momentum.

- The Continuity equation: this equation governs the conservation of mass.

- The Energy equation, and

- The Equation of state

Before representation of governing equations, it should be noted that the SPH formulation follows the Lagrangian basics to define the liquid flow; therefore, all of the governing equations should be adapted to a coordinate system which is rigidly attached to the moving liquid. As a result, the advective expression in the Navier-Stokes equation can be neglected. In this study, the temperature is assumed to be constant; hence, the energy equation is excluded from the governing equations. In SPH methodology the volumetric medium is assumed to be divided into a number of particles; thus, each crucial physical parameter such as density, acceleration, force and mass should be assigned to each of the point nodes.

\subsubsection{Navier-Stokes Equation}

The momentum conservation equation which is adapted to the Lagrangian formulation can take the following form:

$$
\frac{d \vec{v}}{d t}=-\frac{1}{\rho} \nabla P+V_{T}+\vec{F}
$$


In equation (3.10), $\vec{F}$ is the total force acting on the unit mass of liquid, $\vec{v}$ stands for the velocity vector of the fluid motion, $\rho$ defines the liquid density, $P$ is the pressure, and $V_{T}$ is the viscosity of the liquid.

The pressure gradients for the system of particles can be determined by using the gradient approximation of the field function, and can be written as follows:

$$
\nabla P_{i}=\sum_{j} \frac{m_{j}}{\rho_{j}} P_{j} \nabla_{i} W\left(\overrightarrow{r_{\iota \jmath}}, h\right)
$$

Where, $P_{i}$ defines the pressure at any specified point in the liquid domain. To calculate the dynamic pressure induced by a particle at a given point in a given time, the initial pressure including hydrostatic and gravity pressures should be separated from the absolute pressure at that given point; thus, $P_{i}$ is equal to $P_{i}=P_{i}^{a b s}-P_{0} \cdot \overrightarrow{r_{l \jmath}}=\vec{r}_{l}-\vec{r}_{j}$ is a vector which defines the position of point $j$ in comparison with the position of point $i$. Lastly, $h$ is the smoothing length.

The derivative of the ratio of pressure to density can be written as follows:

$$
\frac{\nabla P_{i}}{\rho_{i}}=\nabla\left(\frac{P}{\rho}\right)_{i}+\frac{P_{i}}{\rho_{i}^{2}} \nabla \rho_{i}
$$

Applying the kernel approximation to equation (3.12) gives:

$$
\begin{gathered}
\nabla\left(\frac{P}{\rho}\right)_{i}=\sum_{j} \frac{m_{j}}{\rho_{j}} \frac{P_{j}}{\rho_{j}} \nabla_{i} W\left(\overrightarrow{r_{\imath \jmath}}, h\right) \\
\nabla \rho_{i}=\sum_{j} \frac{m_{j}}{\rho_{j}} \rho_{j} \nabla_{i} W\left(\overrightarrow{r_{\imath \jmath}}, h\right)=\sum_{j} m_{j} \nabla_{i} W\left(\overrightarrow{r_{\imath \jmath}}, h\right)
\end{gathered}
$$

By substituting equations (3.13) and (3.14) into the equation (3.12) with some algebraic simplifications, the pressure gradient can be calculated by the following equation:

$$
\nabla P_{i}=\rho_{i} \sum_{j} m_{j}\left(\frac{P_{i}}{\rho_{i}^{2}}+\frac{P_{j}}{\rho_{j}^{2}}\right) \nabla_{i} W\left(\overrightarrow{r_{\iota \jmath}}, h\right)
$$

The last step is the substitution of the gradient pressure calculated by equation (3.45) into equation (3.11). Consequently, the particle acceleration can be computed by the following equation: 


$$
\overrightarrow{a_{l}}=\frac{d \overrightarrow{v_{l}}}{d t}=-\sum_{j} m_{j}\left(\frac{P_{i}}{\rho_{i}^{2}}+\frac{P_{j}}{\rho_{j}^{2}}\right) \nabla_{i} W\left(\overrightarrow{r_{l \jmath}}, h\right)+V_{T i}+\vec{F}_{l}
$$

In equation (3.16), $V_{T i}$ is a viscous term, and $\vec{F}_{l}$ represents the external forces.

\subsubsection{Continuity Equations}

As mentioned before, the continuity equation represents the conservation of mass during motion, and it has the following form in the SPH formulation:

$$
\frac{d \rho_{i}}{d t}=-\rho_{i} \sum_{j} \frac{m_{j}}{\rho_{j}} \overrightarrow{v_{j}} \cdot \nabla_{i} W\left(\overrightarrow{r_{l j}}, h\right)
$$

In order to rewrite the equation in a similar form to the momentum equation's form and also to reduce the estimation error possibility, the last equation can be rewritten as follows:

$$
\rho_{i} \nabla \cdot \overrightarrow{v_{l}}=\nabla \cdot\left(\rho_{i} \overrightarrow{v_{l}}\right)-\overrightarrow{v_{l} \nabla \rho_{l}}
$$

By substituting equation (3.18) into equation (3.17), the following equations can be obtained:

$$
\begin{gathered}
\nabla \cdot\left(\rho_{i} \overrightarrow{v_{l}}\right)=\sum_{j} \frac{m_{j}}{\rho_{j}} \rho_{j} \overrightarrow{v_{j}} \cdot \nabla_{i} W\left(\overrightarrow{r_{l \jmath}}, h\right) \\
\nabla \rho_{i}=\sum_{j} \frac{m_{j}}{\rho_{j}} \rho_{j} \overrightarrow{v_{\jmath}} \cdot \nabla_{i} W\left(\overrightarrow{r_{l \jmath}}, h\right)
\end{gathered}
$$

Finally, by substituting equation (3.20) into equation (3.17) and considering equations (3.18) and (3.19), the continuity equation in SPH formulation can be denoted as follows:

$$
\frac{d \rho_{i}}{d t}=\sum_{j} m_{j} \overrightarrow{v_{l \jmath}} \cdot \nabla_{i} W\left(\overrightarrow{r_{l \jmath}}, h\right)
$$

$\overrightarrow{v_{l j}}$ is the relative velocity of $i^{\text {th }}$ particle in comparison with $j^{\text {th }}$ particle.

\subsubsection{Equation of State}

The liquid can be assumed to be incompressible. However, artificial compressibility should be added to the SPH formulation to obtain the pressure gradient that is crucial for simulation of the motion of particles. 
Batchelor (1970) proposed the following equation to describe the variation of pressure as a function of density, specifically for the water:

$$
P=B\left(\left(\frac{\rho}{\rho_{0}}\right)^{\gamma}-1\right)
$$

Where, $\gamma=7, \rho$ is the current density of liquid, and $\rho_{0}$ is initial density under the pressure equal to $P_{0}$. B is a constant proportional to the changes in the density of liquid. It should be noted that the compressibility of liquid depends on the square root of Mach number. Constant B can be obtained from the following expression:

$$
B=\frac{\rho_{0} C_{s}^{2}}{\gamma}
$$

Where, $C_{S}$ is the reference speed of sound at a given temperature in the liquid domain and it is proportional to the density of the stored liquid. $C_{s}$ can be computed by following formula:

$$
C_{s}=\frac{V_{f}}{M} \cong \frac{V_{f}}{\sqrt{\eta}}
$$

Where, $\eta=1$ is density related factor, $\mathrm{M}$ is the Mach number, and $V_{f}$ is the highest velocity that the liquid particles can reach under dynamic loading.

\subsubsection{Smoothing Length Calculation}

The performance of each particle is directly associated with its interaction with neighboring particles. There should be an appropriate space in which particles have influence on each other. Smoothing length is a radius of an imaginary sphere which contains the particles that affect the single particle located at the center of that assumed sphere. For each particle one assumed sphere is defined. The smoothing length should be defined at the beginning of the analysis and it is constant through the analysis.

\subsubsection{Viscosity Modelling}

The definition of viscosity in the dynamic fluid-structure interaction simulations needs careful treatment. The fluid viscosity depends on the second derivative of velocity function; however, Monaghan (2005), and Gonzales et al. (2009) implied that the employing of the direct second derivative of viscosity function based on SPH formulation would lead to the instability of 
the numerical estimates, and the violation of the conservation of momentum principle. Many authors have worked on this matter by proposing different types of artificial viscosities. Monaghan (1989) has proposed a satisfactory artificial viscosity as follows:

$$
\begin{gathered}
V_{T i}^{A r t}=\sum_{j} m_{j} \nabla_{i} W\left(\overrightarrow{r_{l \jmath}}, h\right)=\left\{\begin{array}{cc}
\frac{-\alpha \overline{c_{l \jmath}} \mu_{i j}+\beta \mu_{i j}^{2}}{\overline{\rho_{l \jmath}}} ; & \overrightarrow{v_{l \jmath}} \cdot \overrightarrow{r_{l \jmath}}<0 \\
0 ; & \overrightarrow{v_{l \jmath}} \cdot \overrightarrow{r_{l \jmath}}<0
\end{array}\right. \\
\mu_{i j}=\frac{h \overrightarrow{v_{l \jmath}}+\overrightarrow{r_{l \jmath}}}{\overline{r_{l \jmath}^{2}+0.01 h^{2}}}
\end{gathered}
$$

Where, $\overline{\rho_{l \jmath}}$ and $\overline{c_{l j}}$ are the averaged density and the speed of sound between particles, respectively. Moreover, $\alpha$ stands for bulk viscosity, and $\beta$ is assumed to prevent the unrealistic penetration of particles into each other during simulation.

The main role of artificial viscosity is to prevent the instability of simulation in the cases containing liquid motion with high velocity and also for the analysis of free liquid surface motion.

It should be noted that the artificial viscosity in the SPH simulation has the same meaning as bulk viscosity for the finite element method of calculation. Particles use linear and quadratic viscous contribution in order to dampen the high-frequency noises through the analysis.

\subsubsection{Kernel Function}

Liu et al. (2003) first proposed the quartic spline which is used as the kernel function in $\mathrm{SPH}$ numerical simulations. This function with some modifications is mostly used in SPH simulations in most finite element software packages. The quartic spline kernel function is as follows:

$$
W(r, h)=C_{D}\left\{\begin{array}{cc}
\frac{2}{3}-\frac{9}{8}\left(\frac{r}{h}\right)^{2}+\frac{19}{24}\left(\frac{r}{h}\right)^{3}-\frac{5}{32}\left(\frac{r}{h}\right)^{4} & r \leq 2 h \\
0 & r>2 h
\end{array}\right.
$$

Where $C_{D}$ is the normalization constant and is equal to: 


$$
C_{D}=\left\{\begin{array}{c}
\frac{1}{H} \text { for one dimension } \\
\frac{15}{7 \pi h^{2}} \text { for two dimention } \\
\frac{315}{208 \pi h^{3}} \text { for three dimension }
\end{array}\right.
$$

\subsubsection{Density Reinstallation Technique}

The equation of the density (3.17) has a lack of consistency between material properties of the liquid medium such as mass, density, and volume of particles during the period of simulation (Colagrossi and Landrini (2003)). As a result, the density should be established time to time during each simulation. Shepard (1968) introduced a solution based on the interpolation methodology in order to solve this problem as follows:

$$
\rho_{i}=\sum_{j} m_{j} W_{i j}^{*}\left(\overrightarrow{r_{l \jmath}}, h\right)
$$

Where, $W_{i j}^{*}$ is the relative weight and can be written as follows:

$$
W_{i j}^{*}\left(\overrightarrow{r_{\imath \jmath}}, h\right)=\frac{W_{i j}\left(\overrightarrow{r_{l \jmath}}, h\right)}{\sum_{j} W_{i j}\left(\overrightarrow{r_{l \jmath}}, h\right) \frac{m_{j}}{\rho_{j}}}
$$

It should be noted that for three-dimensional problems after each thirty time increments the density should be reinitialized while for two-dimensional problems it should be done after each twenty time increments.

\subsubsection{Time increment Definition}

The period of each time increment in an analysis indicates the time integration of governing equations in a numerical model. The period of each time increment for the analysis depends on several parameters.

Generally, the length of each time increment should be controlled by Courant-FriedrichsLewy (CFL) condition (Couran et al. 1967). The CFL condition is in the following form: 


$$
\Delta t_{1}=\lambda_{1} \frac{h}{C_{s}}=\lambda_{1} \frac{h \sqrt{\eta}}{V_{f}}
$$

The parameter $C_{S}$ stands for the reference speed of the sound. Generally, the speed of the sound dominates the other parameters for indicating the analysis time increment. It should be noted that the higher speed of the sound makes the computational cost higher.

The CFL condition would take the following form providing that the artificial viscosity has to be considered for calculation of time increment:

$$
\Delta t_{2} \leq \lambda_{2} \frac{h}{c_{s}+1.2 \alpha C_{s}+\beta \operatorname{Max}\left(\mu_{i j}\right)}
$$

In this condition, in addition to the speed of the sound, the maximum amount of $\mu_{i j}$ (calculated by Equation (3.25)) among all pairs of nodes in liquid domain is effective.

Moreover, the Morris viscosity term is the other limitation factor for indicating the time increment definition, and it should be controlled as follows:

$$
\Delta t_{3}=\lambda_{3} \frac{h^{2} \rho}{\operatorname{Max}\left(\mu_{i}\right)}
$$

This condition can be neglected, because the liquid medium is homogenous, so there is no difference in the density among all particles in the liquid medium.

The magnitude of the acceleration of the moving liquid is the other limitation factor. This condition should be checked as follows:

$$
\Delta t_{4} \leq \lambda_{i} \sqrt{\frac{h}{\operatorname{Max}\left(a_{i}\right)}}
$$

Lastly, the relative velocity of particles can put restrictions on choosing the appropriate time increment amount:

$$
\Delta t_{5} \leq \lambda_{5} \frac{h}{\operatorname{Max}\left(v_{i j}\right)}
$$


Where, $a_{i}$ and $v_{i j}$ are the maximum acceleration among all particles and the maximum relative velocity among particles interacting with each other, respectively.

It should be noted that based on several related studies, the value of $\lambda_{n}$ varies between 0.05 and 0.4 .

The minimum time increment period which is the result of mentioned conditions will be chosen for time step size for the SPH simulation as follows:

$$
\Delta t=\operatorname{Min}\left(\Delta t_{1}, \Delta t_{2}, \ldots, \Delta t_{5}\right)
$$

\subsubsection{Finite Element Conversion to SPH Particles}

Lagrangian methods are known to be more accurate than the SPH method when the liquid deformation is small, but when the deformation is high and fast, the SPH approach has better performance. In order to take advantage of both the Lagrangian finite element approach and the SPH method in a single modeling simultaneously, the normal finite element method problem can be converted to the SPH problem when the liquid deformation begins to progress.

Normally, the time of that conversion should be specified in each simulation. This feature can reduce the amount of computational effort considerably.

It should be noted that the SPH method has several limitations which should be considered. In this study ABQAUS/CAE is used for simulations, based on the ABAQUS/CAE manual (2016). Those limitations are as follows:

- Generally, it is suggested that the SPH method should be used when other types such as the conventional finite element or coupled Eulerian-Lagrangian methods have reached their fundamental limitations, or they are too expensive to run.

- Surface loads can't be applied on PC3D elements.

- Rough friction should not be defined at the surface interaction for particles.

- Gravity load and mass scaling can't be defined selectively on different materials.

- Element history output is not supported.

- All particles are obliged to be defined by general contact definition.

- 10000 particles should be contained in each domain to achieve good scalability.

- SPH method needs more memory capacity. 


\section{Chapter 5}

\section{Nonlinear Sloshing Response of Rectangular Tanks: FEM Modelling, Results \& Discussion}

\subsection{Introduction}

In this chapter, the Time-History nonlinear analysis of liquid tanks under seismic ground motions is presented. Parametric studies are conducted to investigate the effect of different parameters on the performance of rectangular liquid storage tanks externally excited by seismic dynamic loading. As discussed in previous chapters, the behavior of liquid storage tank systems under dynamic loading is susceptible to a large number of parameters. The main concern of this research is to study the nonlinear sloshing phenomenon. In order to carrying out a consistent research on this subject, different tank plan dimensions, water levels and earthquake records are used for rectangular tank configurations.

In this study, ABAQUS/CAE (Ver. 2016) finite element software is used for modeling and analysis purposes. Nonlinear sloshing modeling technique using SPH formulation is presented under section 5.2.

\subsection{Application of Finite Element Program}

Nonlinearity is required to be considered whenever large amplitude sloshing waves are generated on the liquid free surface. Such waves can contribute significantly to nonlinear distribution of hydrodynamic pressure on liquid free surface as well as along the height of tank's walls. There are only a few numerical techniques in the literature capable of simulating nonlinear sloshing effect in liquid storage tanks externally excited with seismic ground motions. For nonlinear three-dimensional simulation of liquid sloshing there are two different methods available in ABAQUS, namely Coupled-Eulerian-Lagrangian analysis (CEL) and SmoothParticle-Hydrodynamic (SPH). Using any of these two techniques the nonlinear Fluid-StructureInteraction (FSI) effect under dynamic loading can be investigated with high level of accuracy. While CEL is more accurate and provides better visual presentation of final results, it does not 
offer an option to track the sloshing height of the liquid motion through Time-History analysis. This is due to the fact that CEL uses volume fraction approach to model the liquid domain. In this approach, the defined liquid volume can flow through the three-dimensional space defined as an initial condition while the originally defined mesh remains fixed throughout the analysis. As a result, SPH technique remains the only way to track nonlinear liquid sloshing properties of liquid filled tanks subjected to dynamic loading.

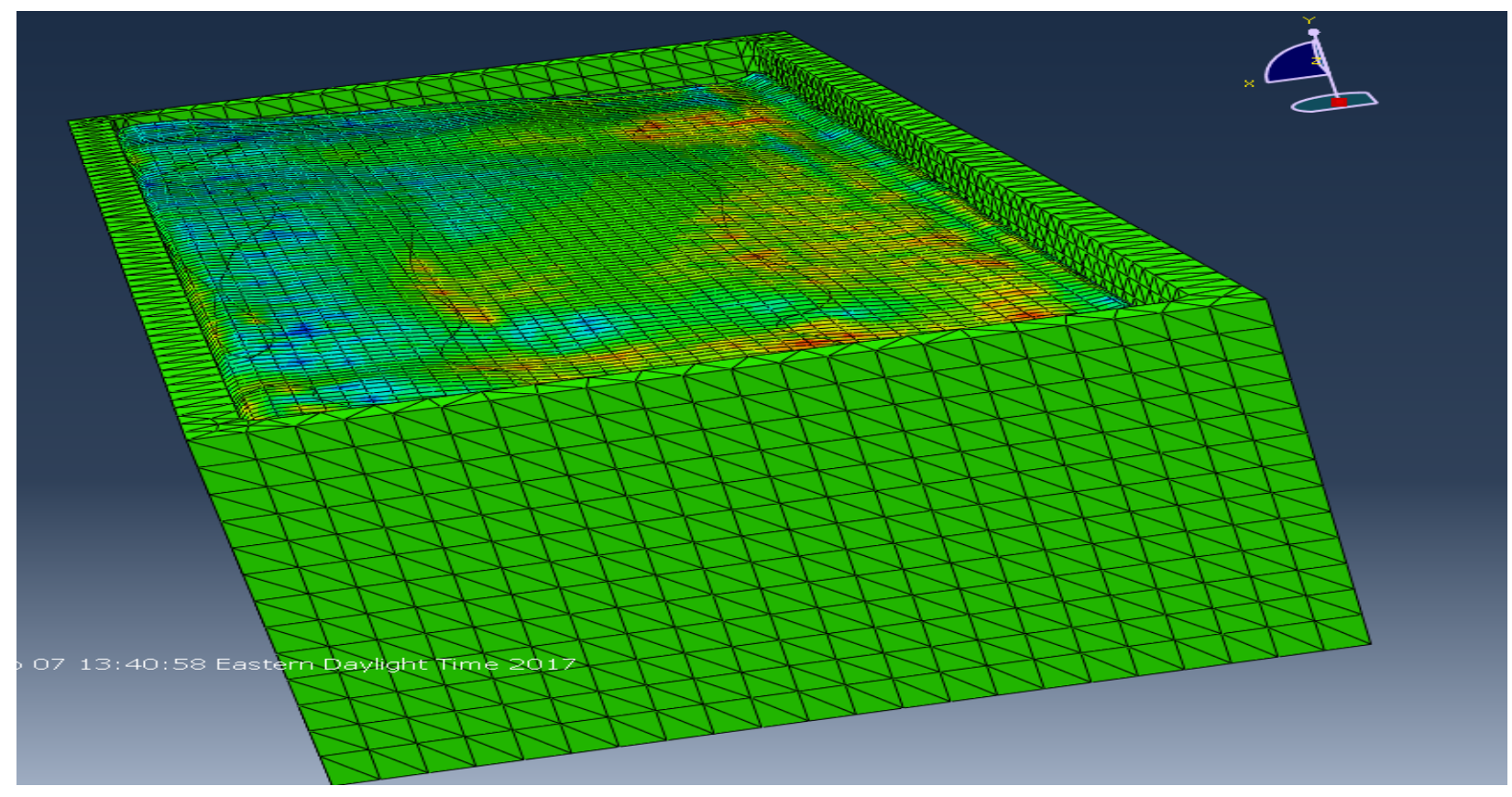

Figure 5.1: Nonlinear sloshing by Coupled Eulerian Lagrangian (CEL) analysis

In this study, the nonlinear wave theory is modeled in a three-dimensional space assuming a geometric nonlinear behavior. Abaqus/Explicit is used for time history analysis because the effect of nonlinear geometry is set as the default in this method. The approach to account for nonlinear wave theory in Abaqus/Explicit is to employ the equations of state. Equations of states are accounted to determine the liquid's volumetric strength in hydrodynamic liquid storage tank simulations; moreover, it determines the pressure as a function of density and specific energy. For this purpose the equation of state is available as Mie-Gruneisen. 
In ABAQUS/CAE, it is possible to employ both SPH and Lagrangian methods in one single model. In fact, a part of model can be simulated as fully Lagrangian while the other part is modeled as Pseudo-Particle. "Finite Element Conversion to SPH Particles Analysis" method is used in this study to model the liquid storage tanks.

Figure 5.1 is showing a typical liquid filled rectangular concrete tank to be studied in this research. The tank is symmetric about $x$ and $z$ directions. Twenty different tank configurations are simulated with ABAQUS/CAE program. The symbols identifying different tank configurations are described in Table 5.1. It should be noted that these names and symbols are adopted from Tavakkoli et al. (2012). In this study, $2 L_{x}$ and $2 L_{z}$ represent the full length of side walls in contact with liquid domain in $x$ and $z$ directions, respectively. $H_{L}$ is the depth of liquid while $H_{w}$ and $t_{w}$ are the height of the tank's wall and thickness of the side walls, respectively. Point $\mathrm{C}$ is located at the corner of the tank at water free surface, and points $\mathrm{A}$ and $\mathrm{B}$ are located at free surface at the middle of side walls perpendicular to $x$ and $z$ directions, respectively.

It should be noted that in order to investigate the effect of tanks' aspect ratios on sloshing, the choice of in which direction the earthquake components are to be applied is not made based on the length of side walls. In fact, the critical horizontal component of the considered records is applied in $X$ direction at all times.

Depending on the ratio of water depth to wall length in $X$ direction, the tanks are divided into two categories, namely "tall" and "shallow". As per Table 5.1, model names starting with $s$ represent shallow while those starting with $T$ represent tall tanks. The two digits following the letter $\mathrm{X}$ indicates the dimension of the tank in plan in $\mathrm{X}$ direction, while the two-digit number after $\mathrm{Z}$ shows the length of the wall parallel to $\mathrm{Z}$ direction. 

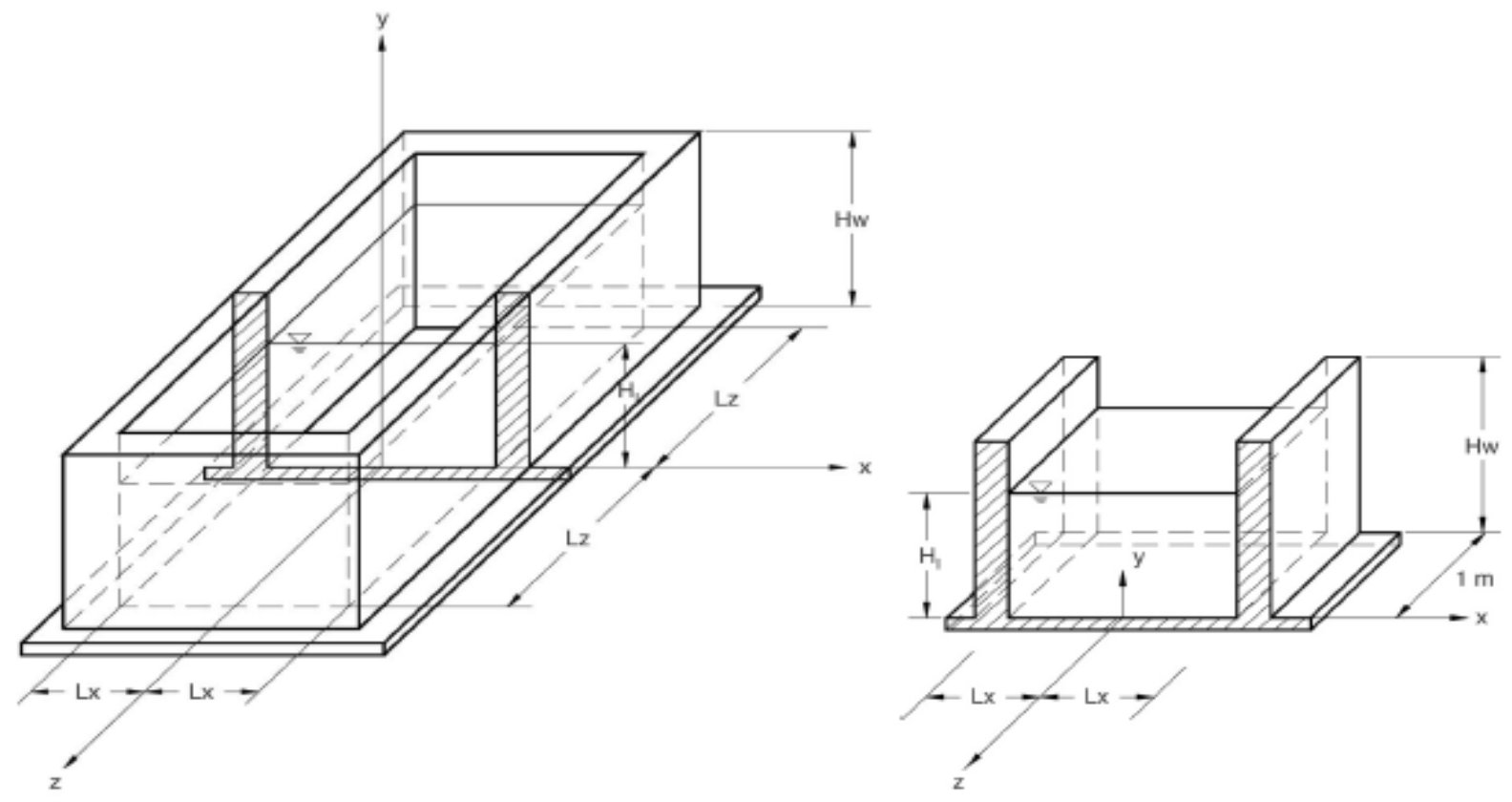

Figure 5.2: Schematic view of a rectangular liquid tank (adapted from Ghaemmaghammi (2010))

Table 5.1: Selected tank models

\begin{tabular}{|c|c|c|c|c|c|c|c|c|}
\hline Types & Groups & Names & $\frac{L_{x}}{L_{z}}$ & $2 L_{x}(\mathrm{~m})$ & $2 L_{z}(\mathrm{~m})$ & $H_{l}(\mathrm{~m})$ & $\frac{H_{l}}{L_{x}}$ & $H_{W}(\mathrm{~m})$ \\
\hline \multirow{6}{*}{ Shallow } & \multirow{2}{*}{ Group 1} & SX50Z25 & 0.5 & 50 & 25 & 7.5 & 0.3 & 8.5 \\
\hline & & SX50Z100 & 2 & 50 & 100 & 7.5 & 0.3 & 8.5 \\
\hline & \multirow{2}{*}{ Group 2} & SX40Z20 & 0.5 & 40 & 20 & 12 & 0.6 & 13 \\
\hline & & SX40Z80 & 2 & 40 & 80 & 12 & 0.6 & 13 \\
\hline & \multirow{2}{*}{ Group 3} & SX30Z15 & 0.5 & 30 & 15 & 15 & 1 & 16 \\
\hline & & SX30Z60 & 2 & 30 & 60 & 15 & 1 & 16 \\
\hline \multirow{4}{*}{ Tall } & \multirow{2}{*}{ Group 4} & TX20Z10 & 0.5 & 20 & 10 & 15 & 1.5 & 16 \\
\hline & & TX20Z40 & 2 & 20 & 40 & 15 & 1.5 & 16 \\
\hline & \multirow{2}{*}{ Group 5} & TX16Z8 & 0.5 & 16 & 8 & 16 & 2 & 17 \\
\hline & & TX16Z32 & 2 & 16 & 32 & 16 & 2 & 17 \\
\hline
\end{tabular}

General material properties used for the finite element modeling of the tanks are as follows: 
Table 5.2: Material properties of the FSI (Fluid-Structure Interaction) system

\begin{tabular}{|c|c|c|c|}
\hline Concrete & $\rho_{c}=2300 \mathrm{~kg} / \mathrm{m}^{3}$ & $E_{c}=2.66 \mathrm{E} 10 \mathrm{~Pa}$ & $v=1.7$ \\
\hline Water & $\rho_{l}=1000 \mathrm{~kg} / \mathrm{m}^{3}$ & $B_{l}=2.10 \mathrm{E} 9 \mathrm{~Pa}$ & \\
\hline
\end{tabular}

Where $\rho_{c}, E_{c}$ and $v$ are density, modulus of elasticity and Poisson's ratio of the tank structure, respectively. Moreover, $\rho_{l}$ and $B_{l}$ are density and bulk modulus of liquid. As the above numbers suggest, the liquid material used for this study is water. However, in order to perform a nonlinear smoothed particle hydrodynamics (SPH) analysis of water, more properties has to be included in the simulation. The liquid is modeled using the $U_{s}-U_{p}$ Hugoniot of the Mie-Gruneisen equation of state. This equation of state uses linear interaction between the shock velocity $\left(U_{S}\right)$ and the particle velocity $\left(U_{P}\right)$;

$$
P-P_{H}=\Gamma \rho\left(E_{m}-E_{H}\right)
$$

Where $P_{H}$ and $E_{H}$ are Hugoniot pressure and specific energy, respectively both of which are merely dependent on material density. $\Gamma$ is the Grneisen ratio defined as;

$$
\Gamma=\Gamma_{0} \frac{\rho_{0}}{\rho}
$$

The Hugoniot energy is related to Hugoniot pressure and can be determined by the following equation;

$$
E_{H}=\frac{P_{H} \eta}{2 \rho_{0}}
$$

Where $\eta=1-\rho_{0} / \rho$ is the nominal volumetric compressive strain. Finally, by substituting the equations (5.2) and (5.3) into the equation (5.1), the equation of state will be derived as follows;

$$
P=P_{H}\left(1-\frac{\Gamma_{0} \eta}{2}\right)+\Gamma_{0} \rho_{0} E_{m}
$$

In linear $U_{s}-U_{p}$ Hugoniot form; 


$$
P_{H}=\frac{\rho_{0} c_{0}^{2} \eta}{(1-s \eta)^{2}}
$$

Where $c_{0}$ and $s$ define the linear relationship between shock velocity and the particle velocity using the following equation;

$$
U_{s}=c_{0}+s U_{p}
$$

By substituting the equations (5.5) and (5.6), the equation of state will be rewritten as follows;

$$
P=\frac{\rho_{0} c_{0}^{2} \eta}{(1-s \eta)^{2}}\left(1-\frac{\Gamma_{0} \eta}{2}\right)+\Gamma_{0} \rho_{0} E_{m}
$$

The equations of state together with the energy equation form a coupled system which is solved for each point within liquid domain for pressure and energy by Abaqus/Explicit. Lastly, as the final step to fully define the characteristics of water, a dynamic viscosity of $0.001 \mathrm{~Pa}$ is applied to the fluid domain.

The tank is assumed to be fixed at the base, and the effects of base uplift and soil flexibility are ignored in this study. The tank structure is meshed using deformable solid elements C3D4; a four-node linear tetrahedron. The liquid domain is also modeled as solid deformable. All the nodes in the simulation have three degrees of freedom (DOF). The structural elements remain as Lagrangian throughout the time-history analysis while the liquid elements are converted to Pseudo-Particles at the beginning of the analysis. In fact, the liquid domain is initially meshed using C3D8R; an eight-node brick element, however it is converted to PC3D particles by the software before the analysis module starts (Figure 5.3). 


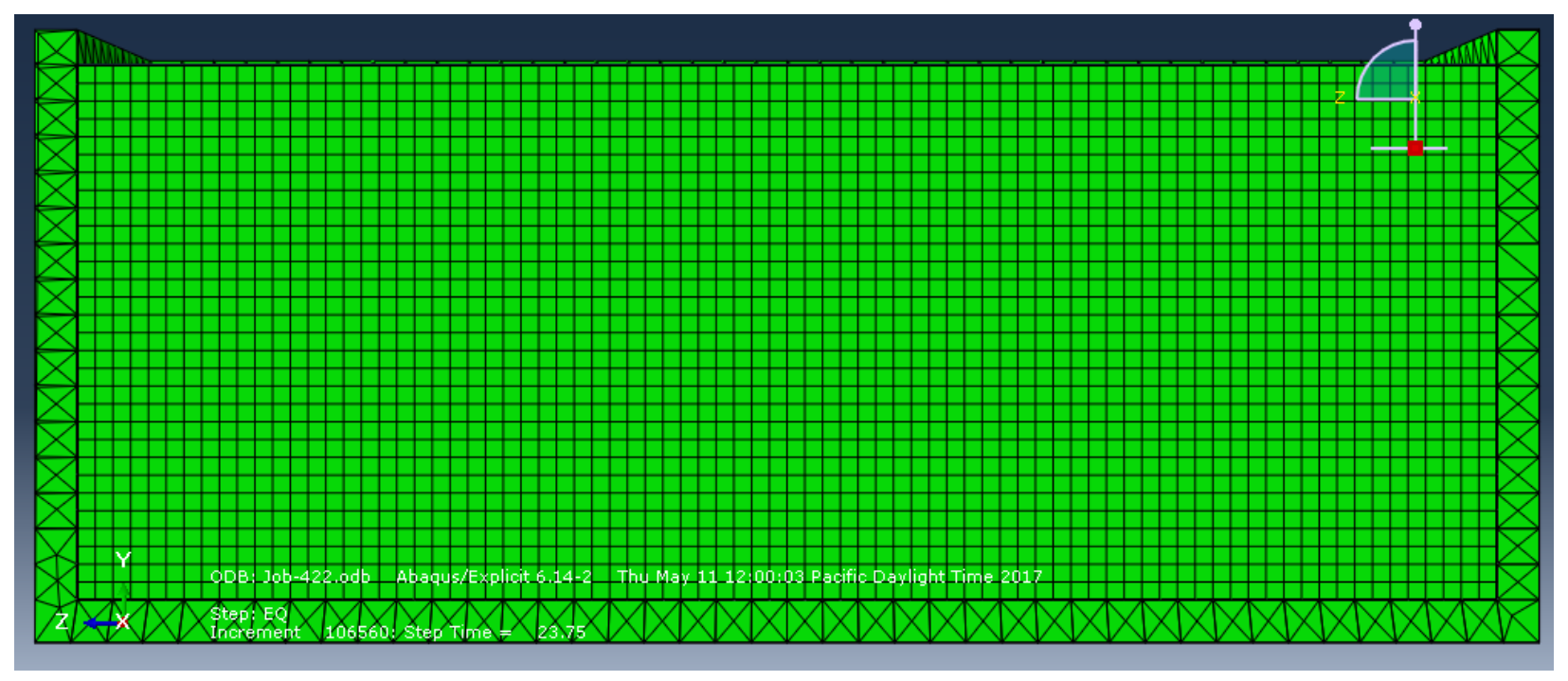

(a)

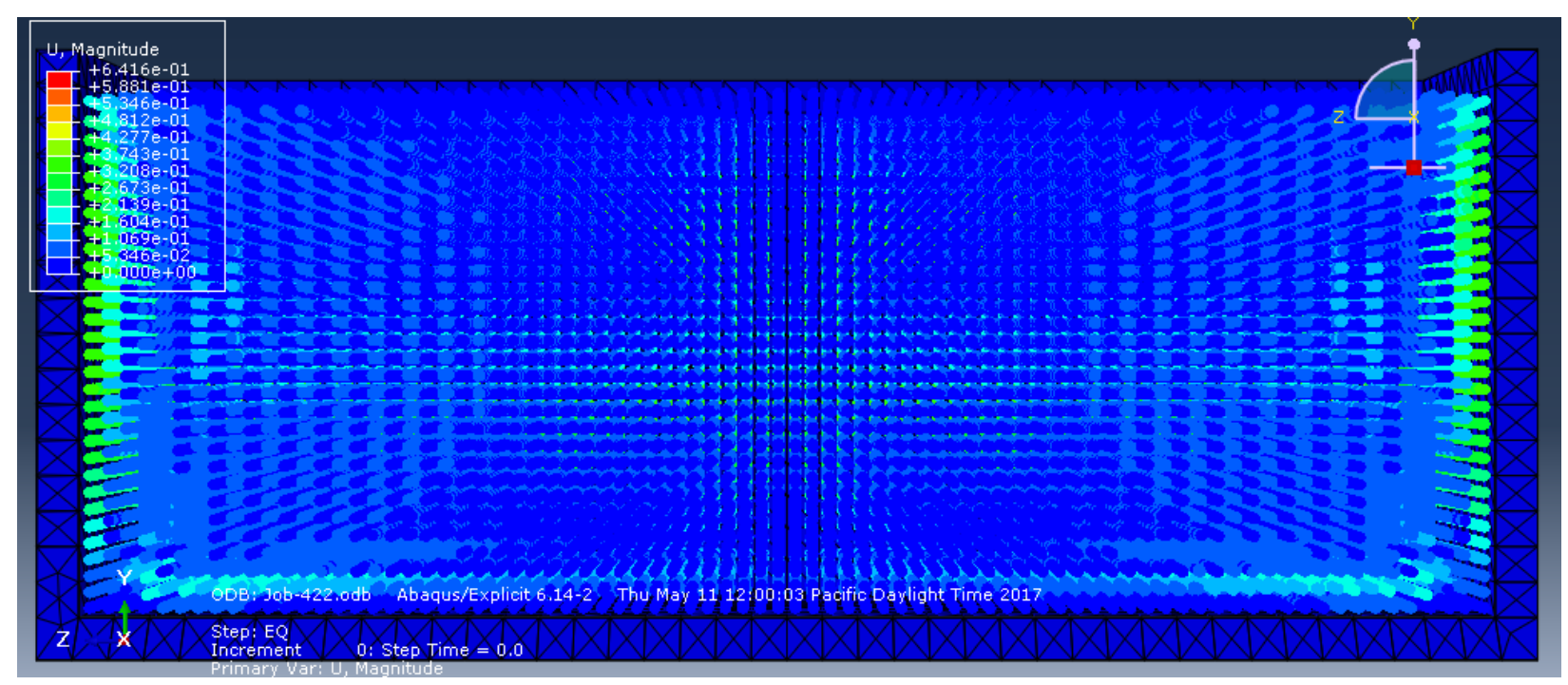

(b)

Figure 5.3: Liquid domain mesh: (a) conventional mesh, (b) pseudo particles

As mentioned before, the tank wall and the base are modeled using tetrahedral shape elements (C3D4). For liquid domain a time-based criterion is used to initiate the conversion from user-defined mesh to SPH particles. By this conversion the continuum element modelling of liquid becomes inactive while the SPH particle simulation option becomes activated. 
The phenomenon of fluid leakage through imperfect sections of the containing structure during analysis is one of the biggest challenges making FSI simulations less accurate or in some cases impossible. In pure SPH modeling in order to prevent from liquid leakage the liquid domain mesh size should be finer than that of the structure. It is well proven that the conversion of continuum to Pseudo Particle mesh reduces the possibility of fluid leakage. This is believed to be one of the most important features of this simulation technique leading to a significant reduction in computational efforts while preserving accuracy and without missing the essential physics of nonlinear FSI phenomenon.

\subsection{Time History Analysis}

In this section, the seismic performance of liquid storage tanks will be investigated. Only horizontal components (Longitudinal and Transverse) of earthquake records are used for time history analysis. The applied seismic load data is in the form of ground motion acceleration with equal time steps. Coupled finite element modeling of the structure and the SPH fluid makes it possible to track down the nonlinear sloshing behavior of the contained liquid.

The recorded data for El-Centro (1940) earthquake is used for seismic excitation of the tank. For all models the N-S component is applied in $X$ direction and the E-W component is applied in $Y$ direction. It should be noted that the components are scaled in such a way that the peak ground acceleration in $X$ direction reaches $0.4 \mathrm{~g}$. The scaled earthquake components of ElCentro (1940) are shown in Figure 5.4; 


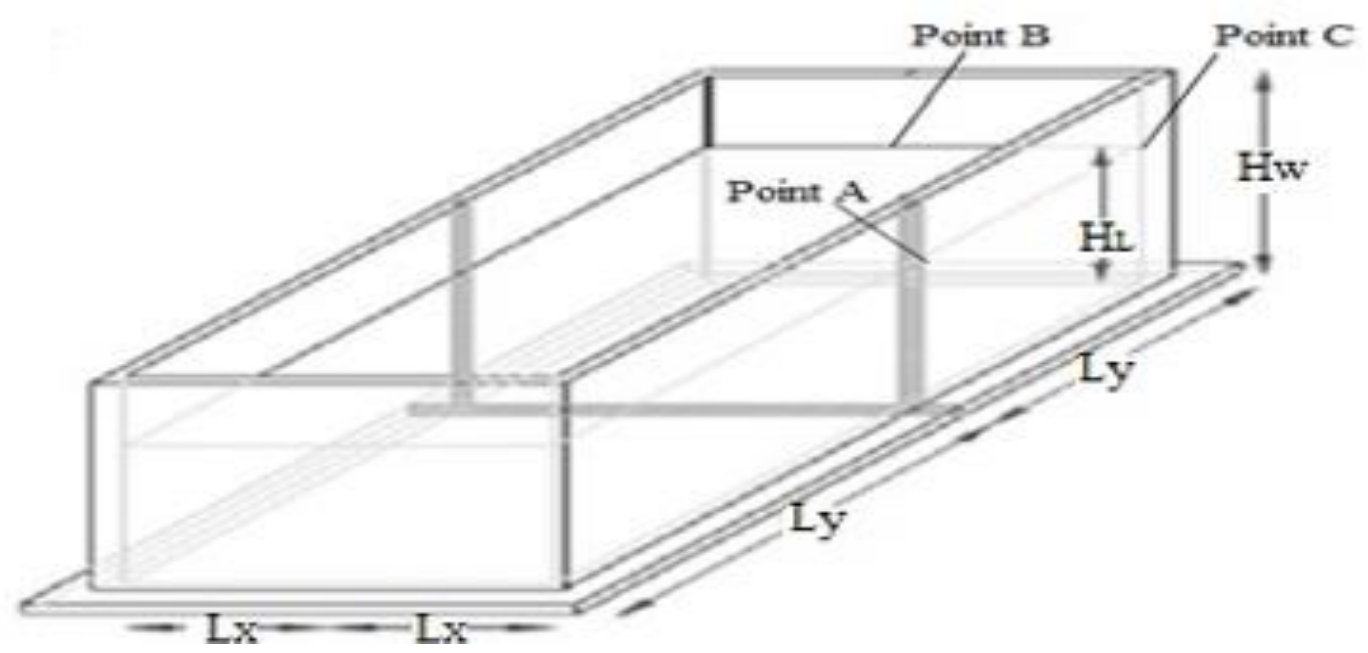

Figure 5.4: Schematic view of tank configurations (I. T. Avval, (2012))

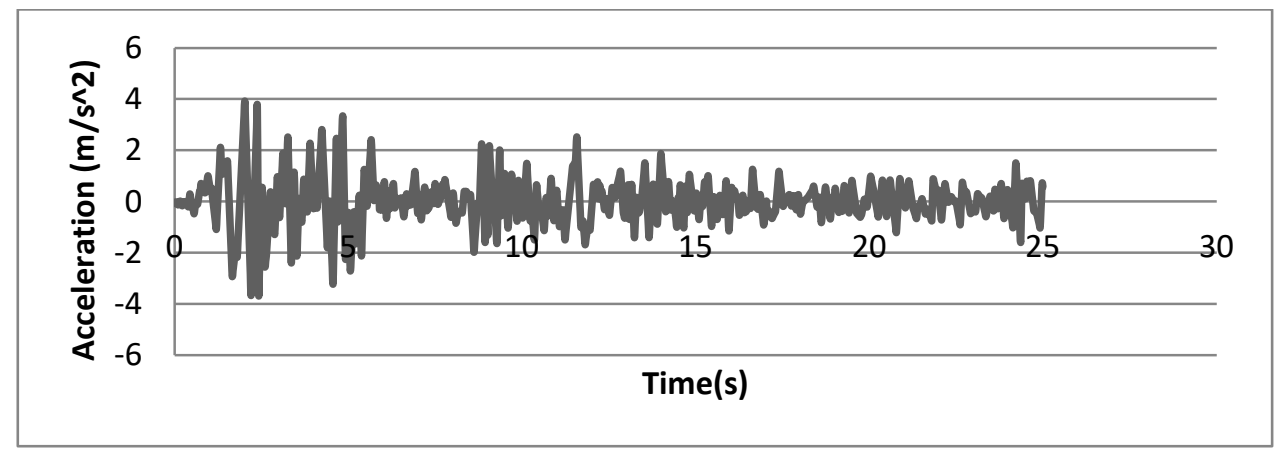

(a)

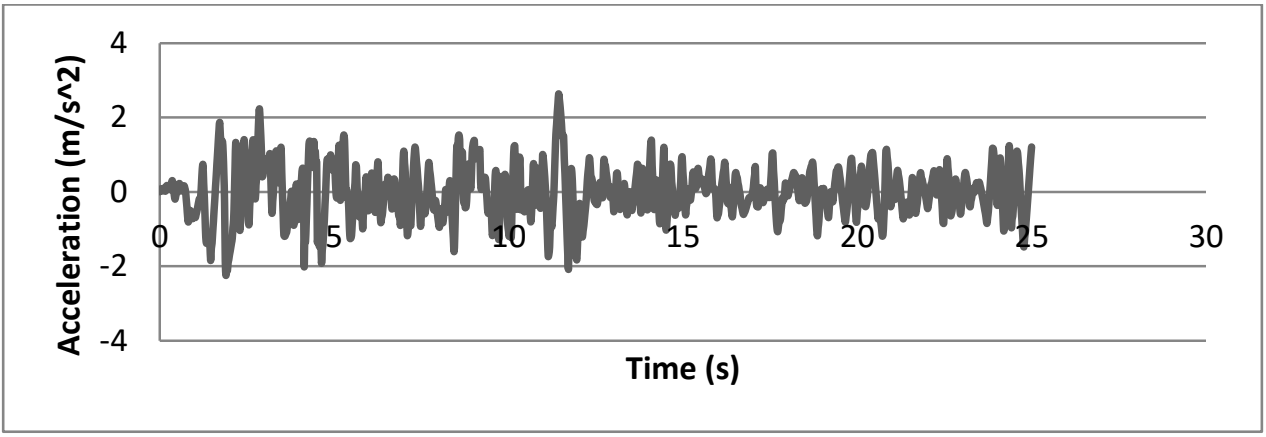

(b)

Figure 5.5: Scaled horizontal components of the 1940 El-Centro record; (a) N-S, (b) E-W 
The sloshing height is measured at different locations on the liquid free surface including the corner of the tank (Point C) as well as the middles of the surrounding walls (Points A and B) as indicated in Figure (5.5).

\subsubsection{Effect of Nonlinearity on Liquid Sloshing Height}

Due to the complexity of the nonlinear sloshing phenomenon, it is recommended to make transition from a simpler model to a more general model. Consequently, as the first step the sloshing behavior of the tanks under unidirectional seismic excitation is studied. For this purpose the N-S component of El-Centro (1940) scaled to the peak ground acceleration of $0.4 \mathrm{~g}$ is applied in $X$ direction (Figure 5.4). The values of maximum sloshing height obtained from FE analysis are summarized in table 5.3. The table provides the maximum sloshing response at different locations on water free surface: Points $A$ and $A^{\prime}$ at the middles of the walls perpendicular to the direction of the ground motion; Point $\mathrm{B}$ at the middle of the wall lying in $\mathrm{x}$ direction; and Point $\mathrm{C}$ at the corner.

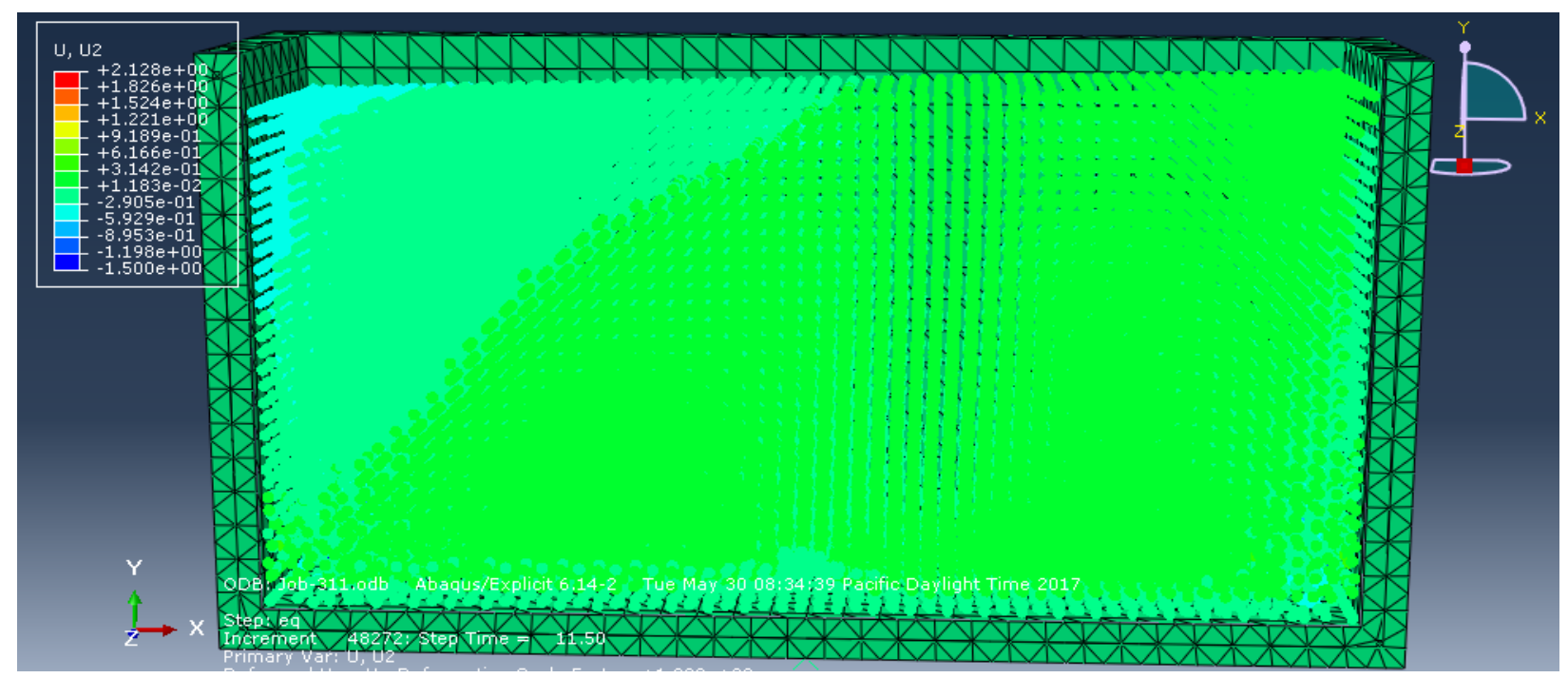

Figure 5.6: Water sloshing in tank model "SX30Z15"

Figure 5.6 is illustrating liquid sloshing inside the tank model "SX30Y15" at $\mathrm{t}=4.25 \mathrm{sec}$ as a result of unidirectional horizontal excitation. The given contour plot is showing the vertical nodal displacement of the whole system. 
Table 5.3: Maximum sloshing height from FE analysis

\begin{tabular}{|c|c|c|c|c|c|c|c|c|c|}
\hline \multicolumn{10}{|c|}{ Maximum Sloshing Height (cm) } \\
\hline Types & Groups & Names & B & $A$ & $A^{\prime}$ & $C$ & $A / A^{\prime}$ & $C / A$ & $T_{c}(s)$ \\
\hline \multirow{6}{*}{$\frac{3}{\frac{3}{\overline{0}}}$} & \multirow{2}{*}{ Group 1} & SX50Z25 & 9.31 & 21.31 & 18.45 & 28.31 & 1.16 & 1.33 & 12.00 \\
\hline & & SX50Z100 & 1.98 & 21.67 & 19.25 & 27.86 & 1.13 & 1.29 & 23.38 \\
\hline & \multirow{2}{*}{ Group 2} & SX40Z20 & 3.66 & 45.63 & 38.71 & 60.19 & 1.18 & 1.32 & 8.30 \\
\hline & & SX40Z80 & 20.00 & 46.24 & 35.81 & 63.88 & 1.29 & 1.38 & 15.18 \\
\hline & \multirow{2}{*}{ Group 3} & SX30Z15 & 9.68 & 39.41 & 34.35 & 47.09 & 1.15 & 1.19 & 6.45 \\
\hline & & SX30Z60 & 6.55 & 40.43 & 32.17 & 51.11 & 1.26 & 1.26 & 10.77 \\
\hline \multirow{4}{*}{$\overline{\bar{\sigma}}$} & \multirow{2}{*}{ Group 4} & TX20Z10 & 7.18 & 38.09 & 32.42 & 43.57 & 1.17 & 1.14 & 5.09 \\
\hline & & TX20Z40 & 7.15 & 38.73 & 33.56 & 45.55 & 1.15 & 1.18 & 7.83 \\
\hline & \multirow{2}{*}{ Group 5} & TX16Z8 & 5.13 & 32.86 & 24.21 & 36.42 & 1.36 & 1.11 & 4.52 \\
\hline & & TX16Z32 & 3.12 & 33.41 & 26.41 & 40.18 & 1.27 & 1.20 & 6.66 \\
\hline
\end{tabular}

The linear sloshing theory suggests that when a single component of ground motion is being applied to a rectangular tank of symmetric configuration, there should be no difference in sloshing height across the entire section of the wall perpendicular to the excitation direction. However, as shown in Table 5.3 the sloshing height at point $C$ representing the corner sloshing is considerably higher than that of point $A$ located at the middle section for all considered models. The obtained results further imply that this corner sloshing effect is more pronounced in Shallow tanks as compared to Tall tanks. While the increasing effect of corner sloshing is estimated to be within $15 \%$ to $36 \%$ for tall models, the range varies from $19 \%$ to $38 \%$ for shallow models. Also given in the table, $T_{c}$ is the fundamental period of sloshing. As expected, tanks with lower aspect ratios (shallow) experience longer periods of vibration in their fundamental convective modes. This is due to the fact that the sloshing response of a typical tank particularly depends on its geometry and dimensions as well as the dynamic specifications of the record.

Therefore, it can be concluded that in the tanks with more liquid depth or less length of the wall parallel to the direction of seismic ground motion the improvement of value of corner sloshing height will be enriched. 


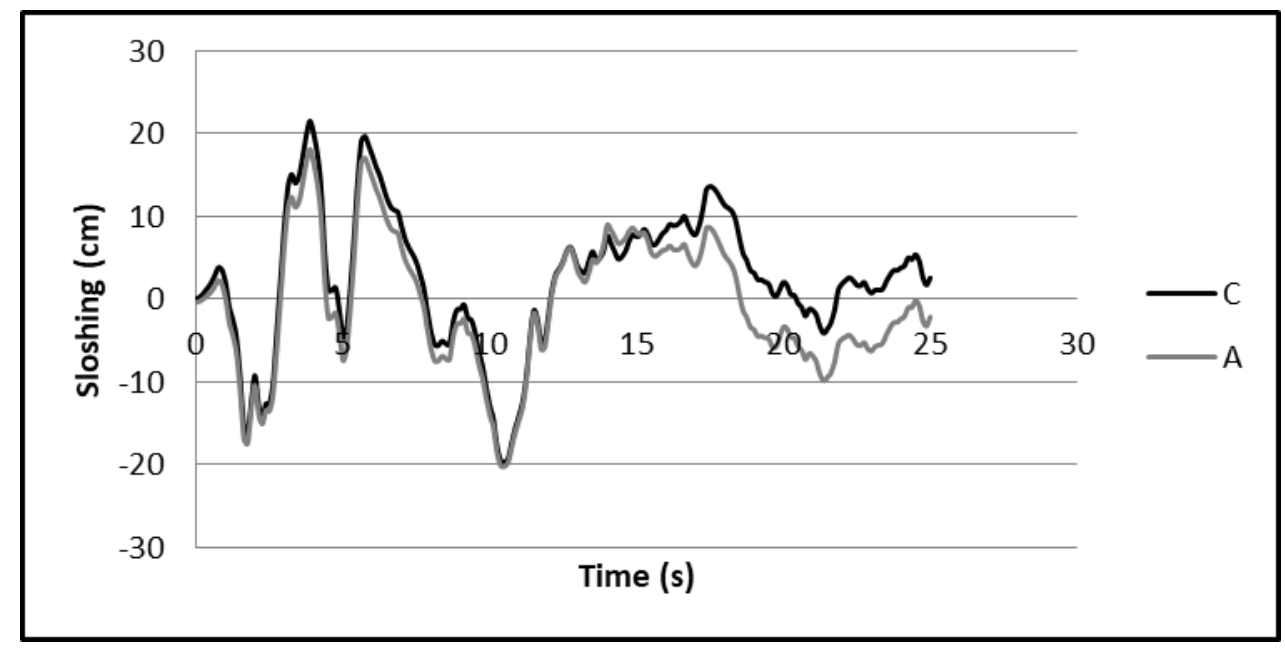

Figure 5.7: Time history of sloshing height for model "SX50Z25"

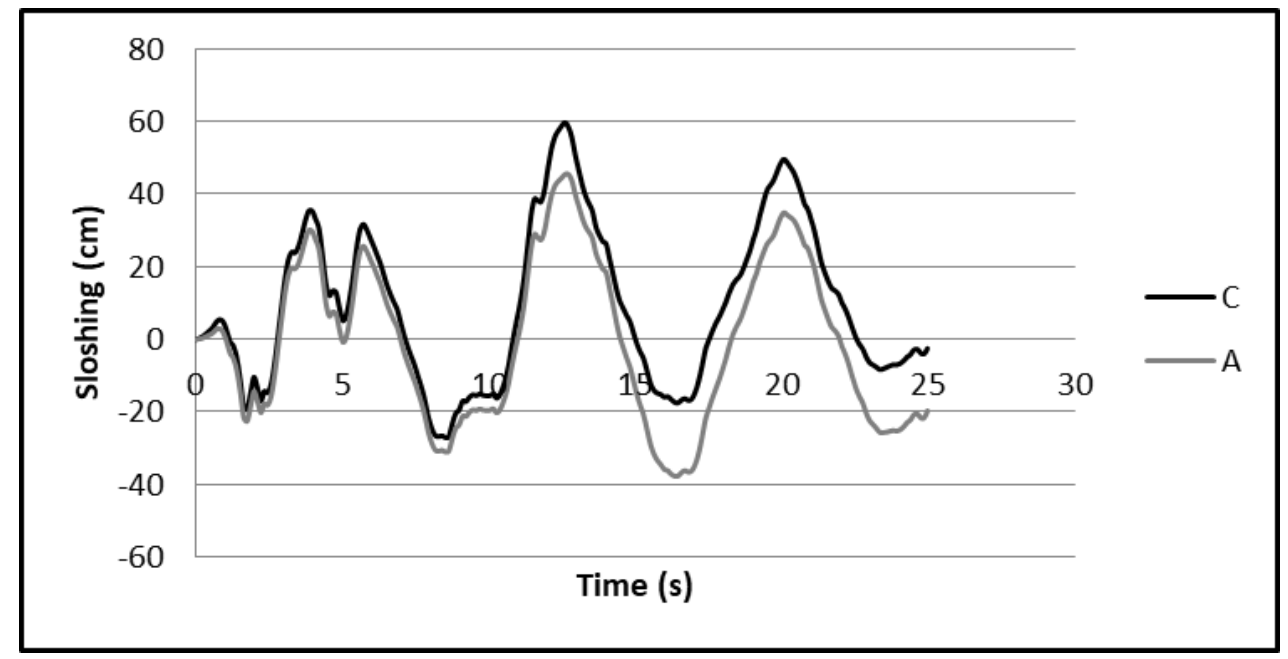

Figure 5.8: Time history of sloshing height for model "SX40Z20" 


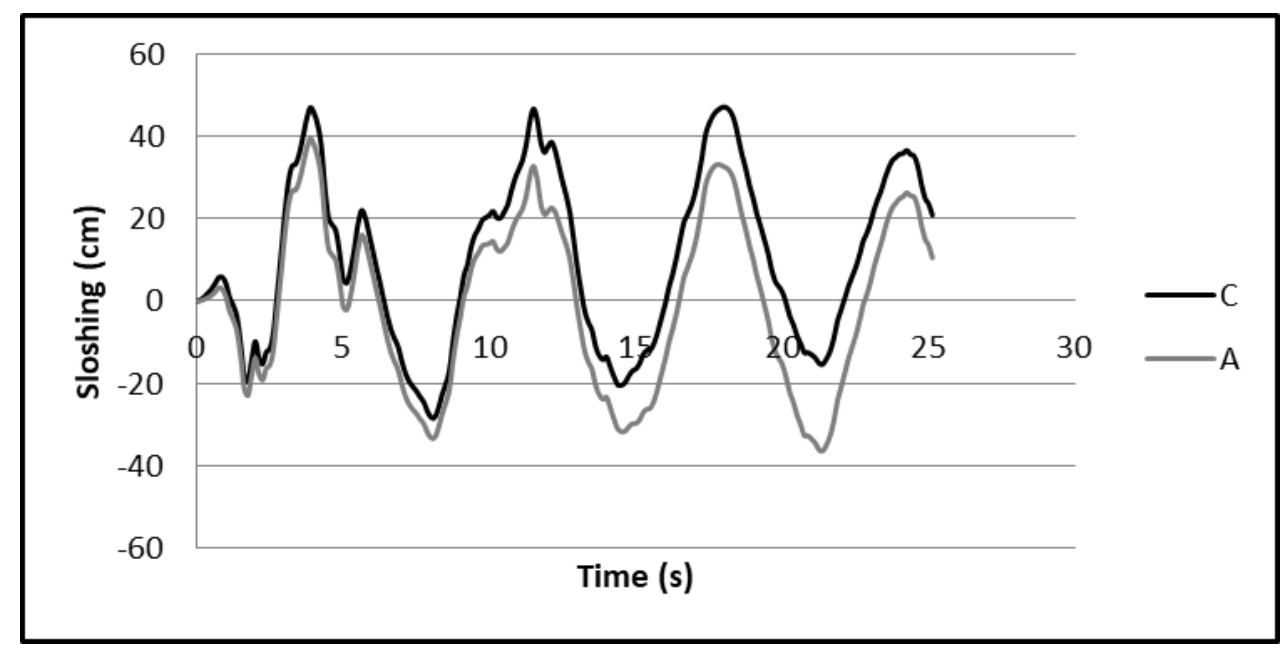

Figure 5.9: Time history of sloshing height for model "SX30Z15"

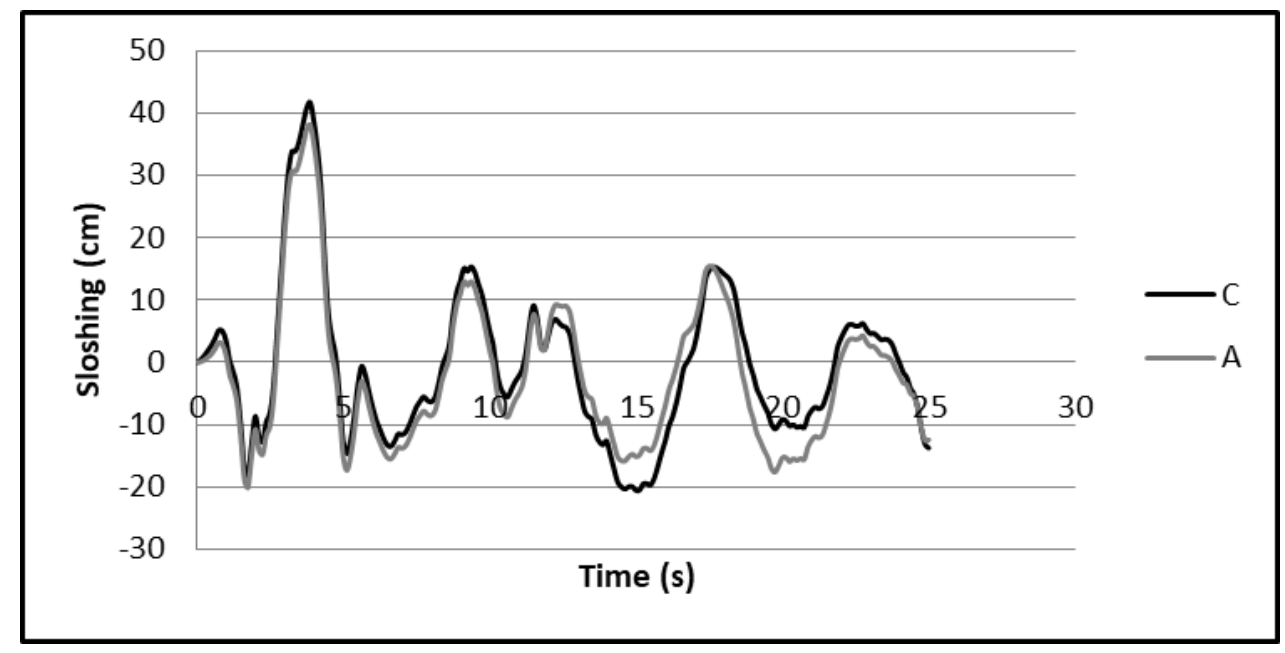

Figure 5.10: Time history of sloshing height for model "TX20Z10" 


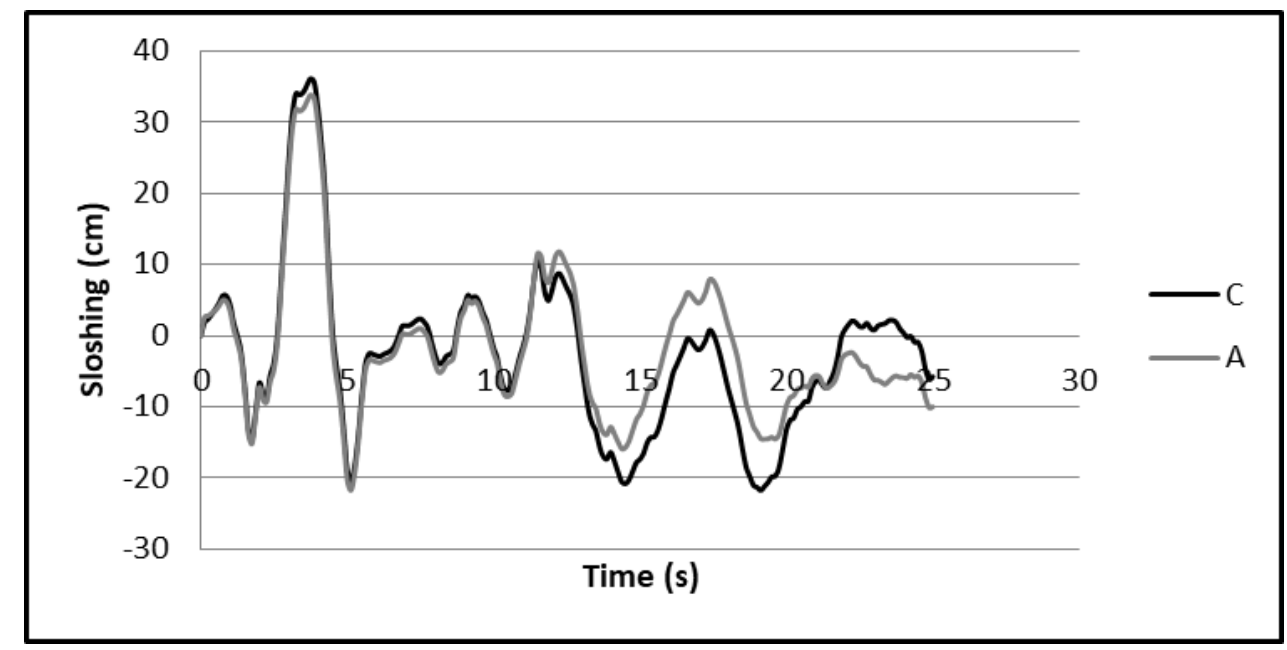

Figure 5.11: Time history of sloshing height for model "TX16Z8"

As can be observed in Figures 5.7 through 5.11, the values of upward corner sloshing are significantly higher compared to those of downward sloshing. A similar trend is also observed at the middle points. The sloshing nonlinearity effect, translating into an excessive corner sloshing consequence is more evident in wider tank models. Finally, the difference between the sloshing response at the corner and at the walls' middle points becomes more noticeable as moving towards the end of the earthquake's duration.

In addition, the linear theory expects that liquid sloshing heights in the middle section of the walls at opposite side of rectangular tanks should be equal; however, nonlinear analysis proposes that the maximum values of sloshing in such locations are not necessarily the same. The sloshing heights on the opposite side walls could differ from $11 \%$ to $20 \%$ in tall tanks, while changing from $19 \%$ to $38 \%$ in shallow tanks. In addition, in linear approach the sloshing height is supposed to be zero at the middle section of the walls parallel to the seismic ground motion while monitoring the sloshing response at Point $B$ suggests that under nonlinear conditions this value is not necessarily zero.

For each group of tanks given in Table 5.1, the aspect ratios of tanks' dimensions in plan change from 0.5 to 2.0. Both tanks under each group have equal lengths in $x$ direction but different lengths in $z$ direction with the length for the second model being 4 times longer than that of the first model. 
The sloshing heights both at $A$ and $C$ points are mostly equal in tanks with different aspect ratio in each group. It is further concluded that the length of the walls perpendicular to the direction of seismic motion has a negligible effect on the maximum sloshing response on the liquid free surface.

As is well-known according to the linear theory, the sloshing response is significantly contributed by the first mode of vibration, and the positive and negative sloshing peaks occure at the opposite side walls perpendicular to the loading direction. Table 5.2 indicates that in general the sloshing periods obtained through a nonlinear FE analysis are lower than the corresponding values from the linear theory mostly known as the Housner's theory adopted in most of the current codes and standards including ACI 350.3-06. However, an opposite trend is observed for one of the models SX40Z20. Also the nonlinear analysis clearly points out that the positive and negative peak response values are not equal. In fact, the positive peak value is always higher than the negative peak. Especially, for tall tanks this difference is almost $20 \%$ while a margin of less than $10 \%$ is observed in shallow tanks.

ACI 350.3-06 estimates the free board for the tanks according to the linear sloshing theory. The free board is needed to be greater than the maximum sloshing height of the liquid free surface in order to prevent the spilling over of the liquid from the tank and to avoid the excessive pressure that might be induced to the tank's roof. As per ACI 350.3-06, the free board of typical rectangular containers is calculated using the following equations:

$$
d_{\max }=\frac{L}{2} C_{c} I
$$

Due to the fact that for all of the tanks in this study $T_{c} \geq \frac{1.6}{T_{s}}=4 \mathrm{sec}$, thus for all cases $c_{c}$ is calculated by the following equation;

$$
C_{C}=\frac{2.4 \times S_{D S}}{T_{C}^{2}}
$$


As mentioned before under Section 3, according to ACI 350.3-06, $T_{C}=\frac{2 \pi}{\sqrt{\lambda}} \sqrt{L}$, where $\lambda=\sqrt{3.16 g \tanh \left(H_{L} / L\right)}$. By substituting these equations into the equation (5.1) and after simplifying, the final equation for $d_{\max }$ for the tanks considered in this work is obtained as;

$$
d_{\max }=0.94 S_{D S} \tanh \left(3.16^{H_{L}} / L\right)
$$

As can be understood from the above equation, the only parameter contributing to the sloshing height for the tanks considered for this study is the ratio of $\frac{H_{L}}{L}$.

By recalling the properties of a standard hyperbolic tangent function, it can be concluded from equation 5.3 that according to ACI 350.3-06, any increase in liquid depth or on the other hand any decrease in wall length parallel to the excitation direction, will directly result in an increase in the maximum sloshing height.

The following figures indicate the sloshing response at Points A and D located on the free surface at the middle of the walls perpendicular to the earthquake direction. Based on the calculated results, maximum sloshing of the liquid free surface takes place at the corner for all the models considered; thus, the design free board should be calculated based on the sloshing response at this point.

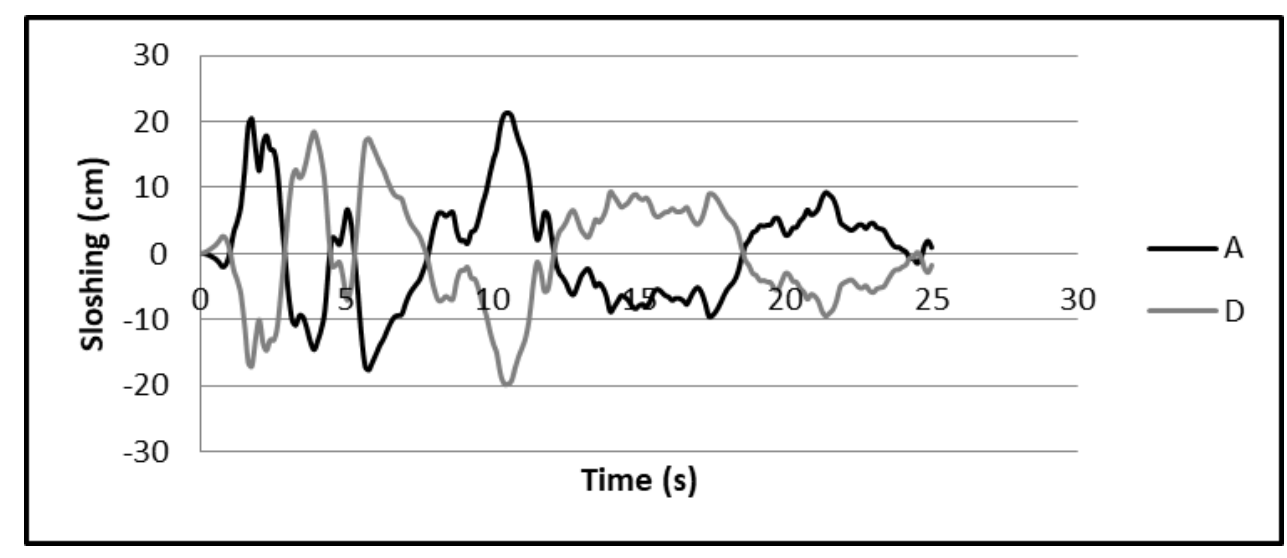

Figure 5.12: Time history of sloshing height for model "SX50Z25" 


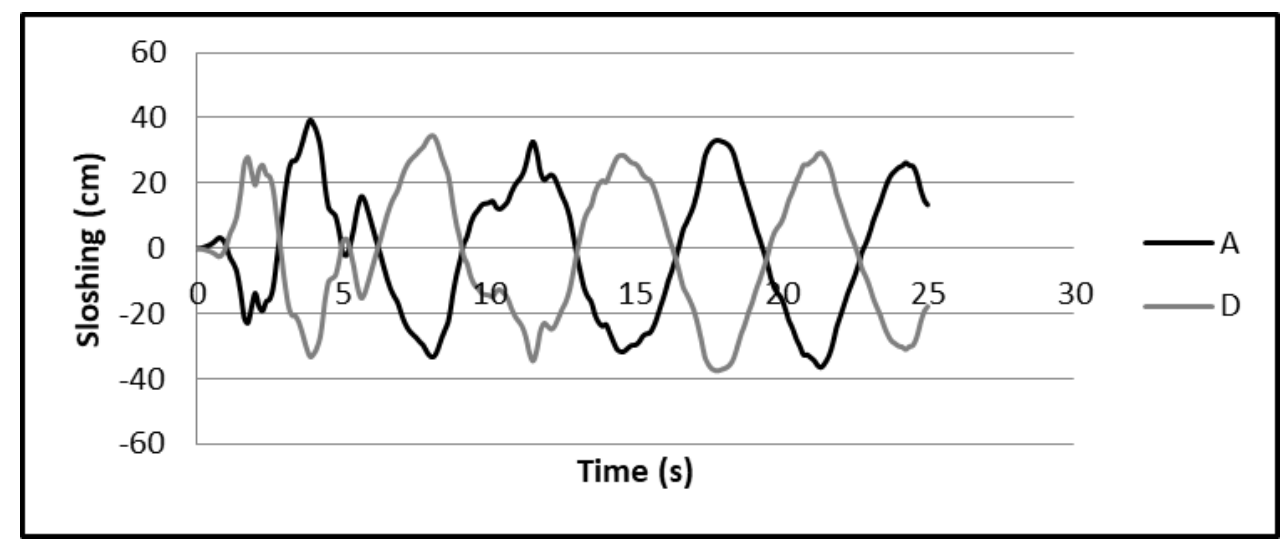

Figure 5.13: Time history of sloshing height for model "SX40Z20"

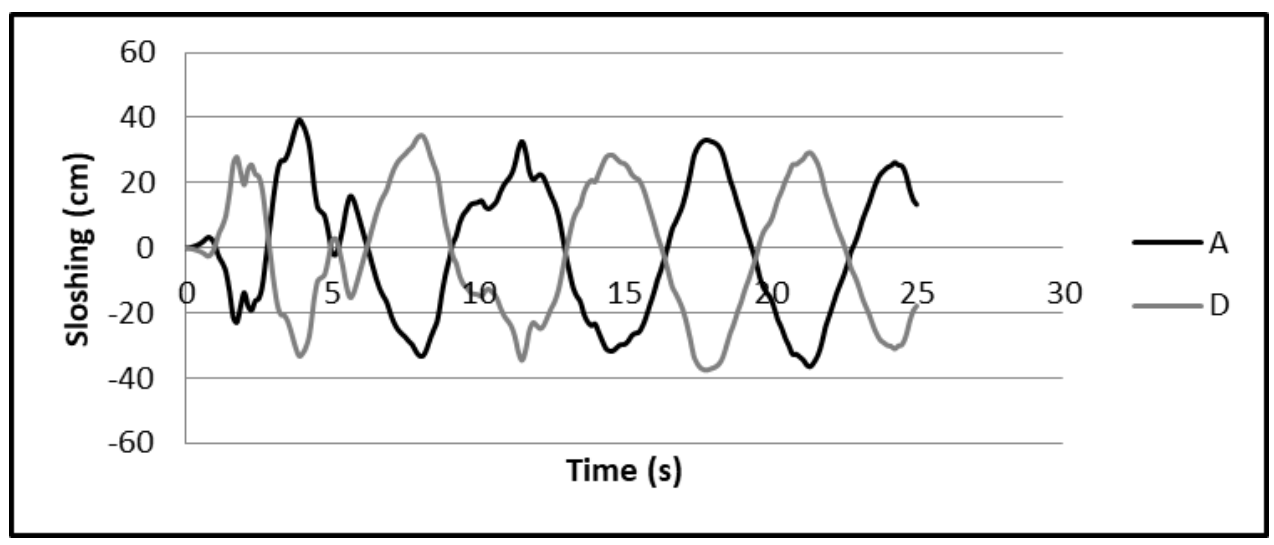

Figure 5.14: Time history of sloshing height for model "SX30Z15"

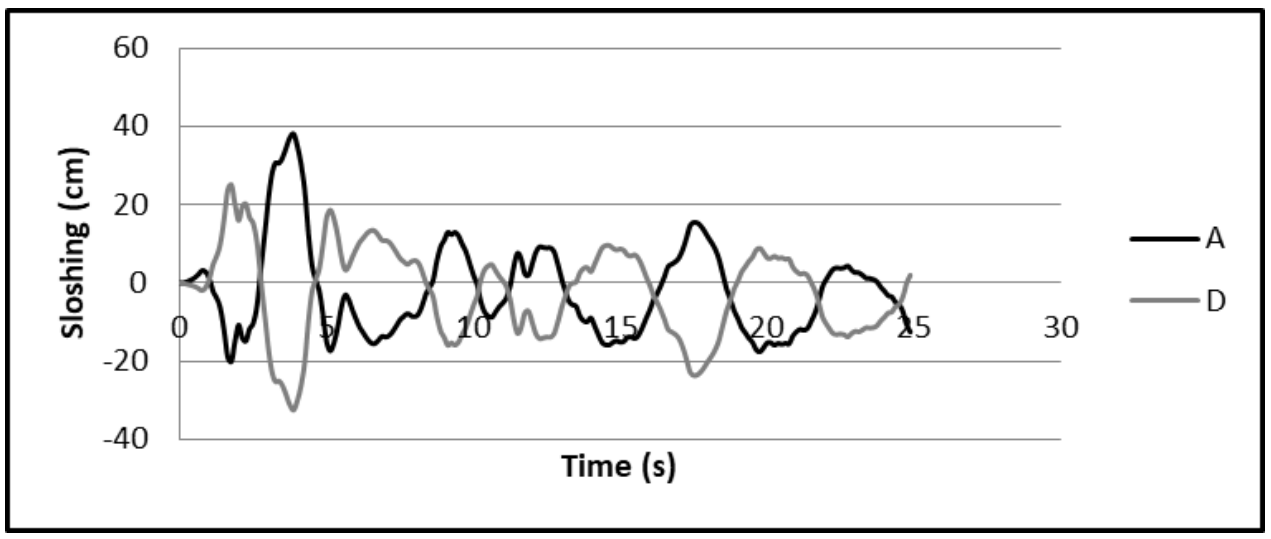

Figure 5.15: Time history of sloshing height for model "TX20Z10" 
According to these figures, the positive sloshing peak is reached at the same time as the negative peak however their values are not the same with positive peak being slightly higher.

Table 5.4: Negative and positive maximum sloshing response

\begin{tabular}{|c|c|c|c|c|c|c|c|}
\hline \multirow{3}{*}{ Types } & \multirow{3}{*}{ Tanks } & \multirow[t]{2}{*}{$\mathrm{ACl}$} & \multirow[t]{2}{*}{ NL-FEM } & \multicolumn{2}{|c|}{$\begin{array}{l}\text { Peak sloshing height } \\
(\mathrm{cm})\end{array}$} & \multirow{3}{*}{$A / D$} & \multirow{3}{*}{$\mathrm{Tc}(\mathrm{ACl}) / \mathrm{tc}(\mathrm{NL})$} \\
\hline & & & & $A$ & D & & \\
\hline & & $\mathrm{Tc}(\mathrm{s})$ & tc $(s)$ & Positive & Negative & & \\
\hline \multirow{3}{*}{ Shallow } & SX50Z25 & 12.00 & 10.50 & 21.33 & -19.80 & 1.08 & 1.14 \\
\hline & SX50Z25 & 8.30 & 12.60 & 45.45 & -42.49 & 1.07 & 0.66 \\
\hline & SX30Z15 & 6.45 & 3.88 & 39.39 & -37.40 & 1.05 & 1.66 \\
\hline \multirow{2}{*}{ Tall } & TX20Z10 & 5.09 & 3.88 & 38.09 & -32.44 & 1.17 & 1.31 \\
\hline & TX16Z8 & 4.52 & 3.75 & 32.87 & -28.52 & 1.15 & 1.21 \\
\hline
\end{tabular}

As shown in Table 5.4, the period of sloshing estimated by the nonlinear finite element analysis is found to be smaller than that from the linear approach suggested in ACI 350.3-06. However, a different trend is observed in model SX50Z25. The calculated results further suggest that there is a greater difference between the obtained values for positive and negative peaks in tall tanks as compared to shallow tanks. Table 5.5 shows a comparison between the maximum sloshing height gained through the nonlinear FE analysis and those recommended by ACI 350.306:

Table 5.5: Comparison between ACI freeboard height and nonlinear FE sloshing

\begin{tabular}{|c|c|c|c|c|}
\hline Types & Groups & Names & $d_{\max }(\mathrm{cm})$ & $\begin{array}{c}\text { NL-Sloshing } \\
(\mathrm{cm})\end{array}$ \\
\hline \multirow{3}{*}{$\frac{3}{\frac{3}{\bar{\pi}}}$} & Group 1 & SX50Z25 & 41.64 & 28.31 \\
\hline & Group 2 & SX40Z20 & 69.69 & 60.16 \\
\hline & Group 3 & SX30Z15 & 86.65 & 47.09 \\
\hline \multirow{2}{*}{$\overline{\bar{\sigma}}$} & Group 4 & TX20Z10 & 92.69 & 43.57 \\
\hline & Group 5 & TX16Z8 & 93.99 & 36.42 \\
\hline
\end{tabular}


Table 5.5 shows that the freeboard height from ACI 350.3-06 is in general higher than that of FE analysis in all cases considered when only a single component of earthquake is present. It should be noted that the ACI350.3 free boards calculated in Table 5.5 is $25 \%$ more than the freeboards which would be the result of ACI 350.3, and the reason is the acceleration data in $X$ direction of El-Centro is scaled with $0.4 \mathrm{~g}$ while the seismic load in scaled with $0.32 \mathrm{~g}$. As stated before ACI 350.3-06 predicts higher freeboards for tanks with higher fluid depths while the nonlinear FE analysis proposes a different trend. The same issue is observed for tanks having different wall lengths. For instance models SX30Z15 and TX20Z10 have both the same water depth but different wall lengths. FE estimates a higher sloshing height for the tank with longer side wall whereas ACI standard predicts otherwise. As mentioned before according to equations (5.1) to (5.3), ACI standard predicts that for the tanks used in this study the only decisive parameter controlling the sloshing response is the ratio of water depth to wall length however the nonlinear FE analysis doesn't agree with this definition. In other words, it can be concluded that the standard hyperbolic tangent function adopted in ACI 350.3-06 does not provide an accurate and adequate picture of sloshing behavior for the tanks subjected to unidirectional seismic motions.

For further clarification, the maximum sloshing height normalized with respect to the height of contained liquid for tanks with different aspect ratios is given in Table 5.4. For comparison purposes, both finite element and ACI results are provided.

Table 5.6: Comparison of nonlinear finite element and code estimations

\begin{tabular}{|c|c|c|c|c|}
\hline$H_{L}(m)$ & $L(m)$ & $H_{L} / L$ & $d_{\max } / H_{L}(\%)$ & $\begin{array}{c}\text { Sloshing/ } \\
H_{L}(\%)\end{array}$ \\
\hline 7.50 & 50.00 & 0.15 & 5.55 & 8 \\
\hline 12.00 & 40.00 & 0.30 & 5.80 & 7 \\
\hline 15.00 & 30.00 & 0.50 & 5.78 & 4 \\
\hline 15.00 & 20.00 & 0.75 & 6.18 & 4 \\
\hline 16.00 & 16.00 & 1.00 & 6.00 & 4 \\
\hline
\end{tabular}


As clearly shown in the table, the normalized sloshing height estimated by the code slightly increases as the aspect ratio approaches 1.00. However, obtained FE results show an inverse relationship between the two variables. In addition, the nonlinear sloshing effect is found to be rather more significant in shallow tanks compared to tall tanks. It is further concluded that the configuration of the tanks in plan has more effect on the nonlinear sloshing behavior than the depth of the contained liquid. Moreover, a more accurate definition than a standard hyperbolic tangent function $(\tanh (x))$ is required in order to adequately estimate the maximum sloshing height in rectangular liquid containers.

\subsubsection{Nonlinear Liquid Sloshing under Bi-directional Seismic Ground Motions}

In reality, a liquid storage tank excited by a seismic event absorbs the components of earthquake acceleration both in horizontal (longitudinal and transverse) and vertical directions. Previous studies on the subject imply that the vertical acceleration has no significant effect on the sloshing response; therefore, in this study the effect of vertical acceleration is ignored. In this section, the simultaneous application of horizontal components of El-Centro record is examined. It should be noted that the components are scaled in such a way that the peak ground acceleration of the N-S component becomes equal to $0.4 \mathrm{~g}$.

For all models considered throughout this section, it is assumed that the N-S and E-W components of the record are applied in $x$ and $z$ directions, respectively. For the tanks in the first

group with $\frac{L_{x}}{L_{z}}=2.0$, the longer side wall lies in $x$ direction conversely for the second group with $\frac{L_{x}}{L_{z}}=0.5$, the shorter wall is aligned in $x$ direction.

In tanks excited externally with two seismic motions acting simultaneously, the interference of these waves will lead to a totally different sloshing pattern on the liquid free surface as compared to the case of unidirectional action. In situations where the sloshing response modes resulting from the two waves are in phase with each other, the bidirectional effect could yield to a considerable increase in the total response. On the other hand, if the two waves create out-of-phase oscillations of the free surface, including such effect could lead to some reduction in the total response. In this study, the liquid sloshing height at three points located on the free surface is investigated; Point $\mathrm{C}$ at the corner of the tank, Point $\mathrm{A}$ at the middle section of the wall parallel to $\mathrm{Z}$ direction, and Point $\mathrm{B}$ at the middle section of the wall parallel to 
$\mathrm{X}$ direction. In Table 5.5 the liquid sloshing height at these points is presented. It should be noted that for each case the listed corner sloshing value is the maximum upward sloshing height among all four corner points of the tank. For middle points, the listed value is the maximum upward sloshing height on either side of the tank for the direction under consideration.

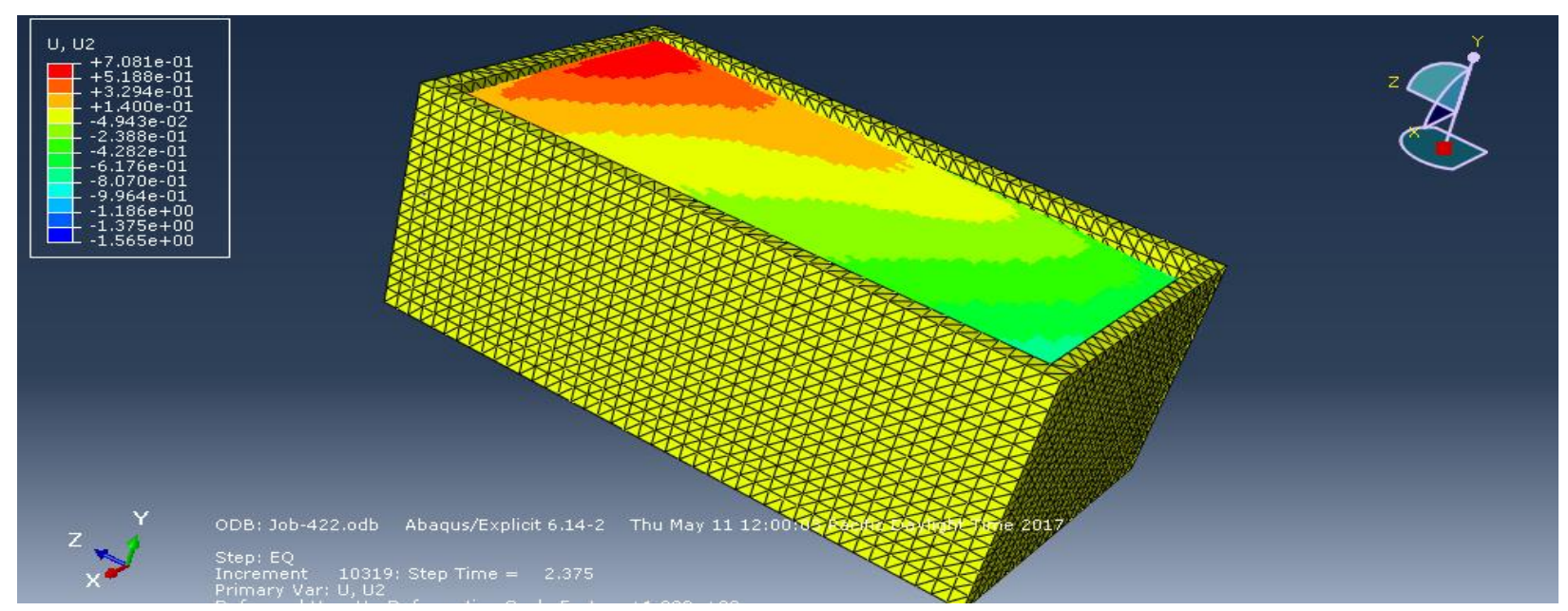

(a)

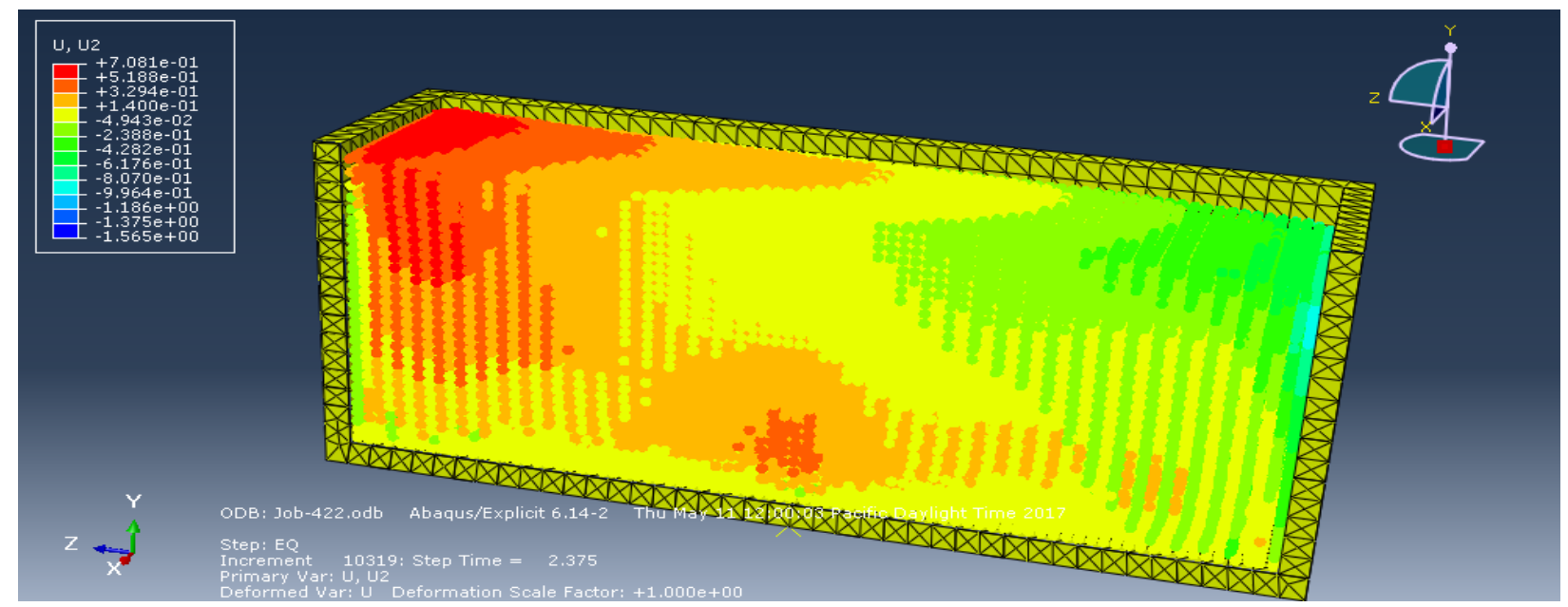

(b)

Figure 5.16: Liquid sloshing contour for TX20Y40: (a) whole model (b) sectional view through the tank 
Table 5.7: Maximum liquid sloshing height under bi-directional seismic ground motions

\begin{tabular}{|c|c|c|c|c|c|c|c|}
\hline \multicolumn{8}{|c|}{ Maximum Sloshing Height (cm) } \\
\hline Types & Groups & Names & $H_{L}(m)$ & $H_{L} / L$ & $C$ & $A$ & $B$ \\
\hline \multirow{6}{*}{$\frac{\frac{3}{0}}{\frac{\sqrt{\sigma}}{\tilde{N}}}$} & \multirow{2}{*}{ Group 1} & SX50Z25 & 7.50 & \multirow{2}{*}{0.15} & 57.59 & 19.51 & 53.76 \\
\hline & & SX50Z100 & 7.50 & & 69.73 & 21.65 & 59.67 \\
\hline & \multirow{2}{*}{ Group 2} & SX40Z20 & 12.00 & \multirow{2}{*}{0.30} & 88.24 & 45.11 & 45.36 \\
\hline & & SX40Z80 & 12.00 & & 93.26 & 46.97 & 64.49 \\
\hline & \multirow{2}{*}{ Group 3} & SX30Z15 & 15.00 & \multirow{2}{*}{0.50} & 59.37 & 39.37 & 37.09 \\
\hline & & SX30Z60 & 15.00 & & 82.26 & 41.82 & 70.18 \\
\hline \multirow{4}{*}{$\overline{\overline{\mathscr{D}}}$} & \multirow{2}{*}{ Group 4} & TX20Z10 & 15.00 & \multirow{2}{*}{0.75} & 62.29 & 39.37 & 24.37 \\
\hline & & TX20Z40 & 15.00 & & 108.42 & 39.90 & 67.49 \\
\hline & \multirow{2}{*}{ Group 5} & TX16Z8 & 16.00 & \multirow{2}{*}{1.00} & 62.29 & 36.07 & 25.80 \\
\hline & & TX16Z32 & 16.00 & & 93.97 & 37.66 & 81.20 \\
\hline
\end{tabular}

Based on the linear theory of sloshing, it is expected that for the tank sizes considered in this study the sloshing height gets higher as the ratio of $\left(H_{L} / L\right)$ reaches higher values by moving from shallow to tall tanks. However, as can be observed the calculated results from nonlinear FE analysis suggest a different fashion. For instance, the length of the wall in TX16Y8 is $25 \%$ shorter than that of TX20Y10, while both models have almost the same liquid depth. FE analysis predicts similar sloshing heights at Point $C$ for both models whereas the linear approach estimates a higher sloshing response for TX16Y8.

By comparing the results, it also becomes clear that the dimensions of the tanks in plan have more effect on the liquid sloshing height than the depth of the liquid especially in shallow tanks. The importance of this effect is underestimated in the linear approach. The obtained FE results further suggest that although the plan dimension effect is more observable in shallow tanks, its impact could be still considerable in tall tanks.

For further clarification, some additional data on the effect of bi-directional excitation on the sloshing behavior of the tanks is provided in Table 5.8. In this table, the value of maximum sloshing height at the corner is normalized with respect to its value at the middles of the surrounding walls. 
Table 5.8: Normalized sloshing height values

\begin{tabular}{|c|c|c|c|c|c|c|}
\hline Types & Groups & Names & $H_{L}(m)$ & $H_{L} / L$ & $C / A$ & $C / B$ \\
\hline \multirow{6}{*}{$\begin{array}{l}\frac{3}{0} \\
\frac{\bar{V}}{\sqrt{0}} \\
\frac{c}{n}\end{array}$} & \multirow{2}{*}{ Group 1} & SX50Z25 & 7.50 & \multirow{2}{*}{0.15} & 2.95 & 1.07 \\
\hline & & SX50Z100 & 7.50 & & 3.22 & 1.17 \\
\hline & \multirow{2}{*}{ Group 2} & SX40Z20 & 12.00 & \multirow{2}{*}{0.30} & 1.96 & 1.95 \\
\hline & & SX40Z80 & 12.00 & & 1.99 & 1.45 \\
\hline & \multirow{2}{*}{ Group 3} & SX30Z15 & 15.00 & \multirow{2}{*}{0.50} & 1.51 & 1.60 \\
\hline & & SX30Z60 & 15.00 & & 1.97 & 1.17 \\
\hline \multirow{4}{*}{$\overline{\bar{\sigma}}$} & \multirow{2}{*}{ Group 4} & TX20Z10 & 15.00 & \multirow{2}{*}{0.75} & 1.58 & 2.56 \\
\hline & & TX20Z40 & 15.00 & & 2.72 & 1.61 \\
\hline & \multirow{2}{*}{ Group 5} & TX16Z8 & 16.00 & \multirow{2}{*}{1.00} & 1.73 & 2.41 \\
\hline & & TX16Z32 & 16.00 & & 2.50 & 1.16 \\
\hline
\end{tabular}

Although there is no provision in ACI 350.3-06 for considering the effect of corner sloshing, few studies have been conducted on the subject such as Kianoush et al. (2010). As shown by yellow cells in the table, for the following discussion the corner sloshing response is compared with that at the middle section of the shorter wall in plan. As mentioned before, in this study N-S component of the record is applied in $X$ direction, and E-W component is applied in $Z$ direction. For better understanding of the impact of the tank plan configuration on the response rather than the effect of the record's amplitude itself, for the tanks with $\left(L_{x} / L_{z=}\right)$ the stronger component is applied in the longer direction while for the second group with $\left(L_{x} / L_{z=} 0.5\right)$ the stronger component is applied in the shorter direction. As evident from the table, for the models in the second group, the liquid sloshing has reached higher values at Point $B$ compared to Point $A$. Considering the fact that the weaker component is pointed at $B$, it is expected that sloshing at this point be lower opposite to the trend the obtained FE results are showing. This reveals the fact that the tank plan configuration has more determinative effect on the sloshing behavior than the amplitude of the earthquake component.

It is further concluded that the corner sloshing effect is more significant in shallow tanks compared to tall tanks. 
To focus more on the effect of the tank plan configuration, a comparison is made between the sloshing behavior of the tanks with the same water depth but different plan dimensions, as listed in Table 5.9.

Table 5.9: Comparison of the sloshing behavior of the tanks with equal water depths

\begin{tabular}{|c|c|c|c|c|c|}
\hline \multicolumn{6}{|c|}{ Ratio of Maximum Sloshing Height at Different Locations } \\
\hline Types & Groups & $H_{L} / L$ & $C / C$ & $A / A$ & $B / B$ \\
\hline \multirow{3}{*}{ Shallow } & 1 & 0.15 & 1.21 & 1.11 & 1.11 \\
\cline { 2 - 6 } & 2 & 0.30 & 1.06 & 1.04 & 1.42 \\
\cline { 2 - 6 } & 3 & 0.50 & 1.39 & 1.06 & 1.89 \\
\hline \multirow{3}{*}{ Tall } & 4 & 0.75 & 1.74 & 1.01 & 2.77 \\
\cline { 2 - 6 } & 5 & 1.00 & 1.51 & 1.04 & 3.15 \\
\hline
\end{tabular}

In this table, the maximum sloshing heights in models with $L_{x} / L_{y}=0.5$ are normalized with respect to the corresponding values of those with $L_{x} / L_{y}=2.0$. In each group the water depth and the length of the wall parallel to $\mathrm{X}$ direction are considered to be equal thus, the only difference remains to be the wall length in $Z$ direction. The linear approach predicts that the sloshing height should be lower in the wider tanks.

in all cases the sloshing height is higher in such tanks. The ratios here show the importance of the effect of the tank configuration in plan. One more time the nonlinear FE analysis proves the point that the function $\tanh \left(3.16 H_{L} / L\right)$ cannot provide an accurate estimation of the maximum sloshing height.

By paying a closer attention at the variations of response at Point $\mathrm{A}$, it can be observed that the sloshing response does not significantly change at this point. The length of the wall parallel to $\mathrm{X}$ direction is the same in both tank types. Therefore, the sloshing height at the middle section is independent of the length of the wall in the perpendicular direction.

Sloshing heights at Point B are boosted in all of the tanks. This clearly shows the key role the length of the wall can play. The sloshing heights in tanks with higher $H_{L} / L$ values are boosted more. As mentioned before, for the tanks with sloshing periods longer than 4 seconds 
which is the case in this study, ACI standard predicts a direct relationship between the maximum sloshing height and $\left(H_{L} / L\right)$ ratio. However, the nonlinear analysis shows an inverse relationship between the two, meaning that a decrease in the aspect ratio results in an increase in the maximum sloshing response for all cases considered.

Figures 5.18 to 5.27 present the time history of liquid sloshing of all the models. It should be noted that the N-S component of El-Centro record has a higher peak amplitude and is therefore considered to be more critical than the E-W component. For the first five models with $\left(L_{x} / L_{z}=2\right)$ this critical component is applied in the longer direction parallel to $\mathrm{x}$ axis (critical direction).

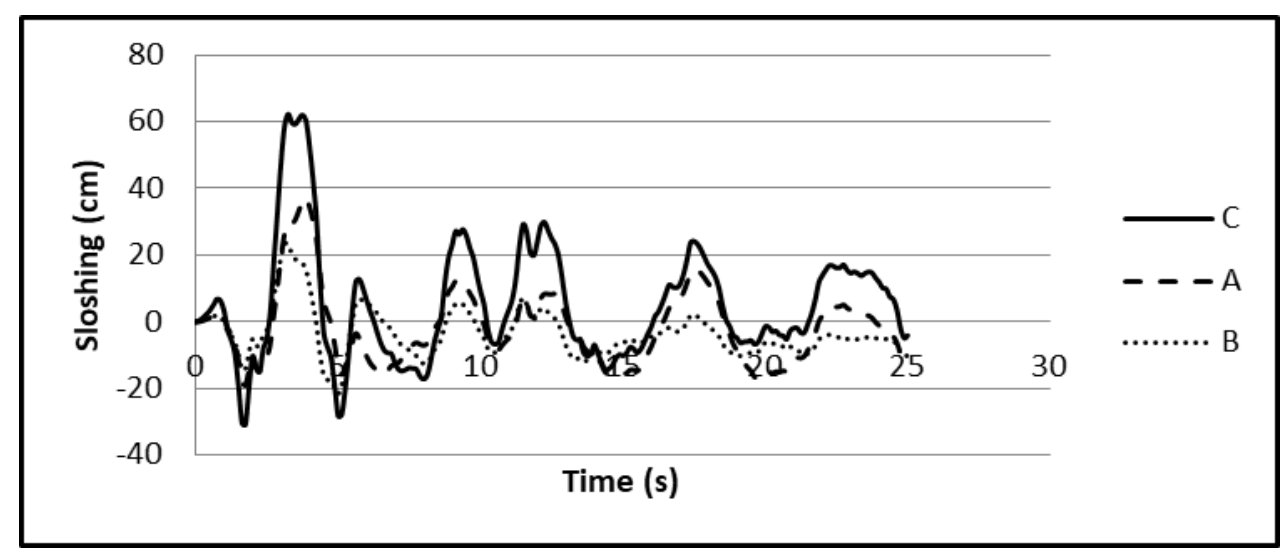

Figure 5.17: Time history of sloshing height for model "TX16Z8"

Figure 5.17 represents the time history of sloshing height for the most slender tank "TX16Y8" in this research. It is seen that for all three points the maximum sloshing occurs at the same time after 3.5 seconds from the beginning of the analysis. The corner sloshing is found to be about $75 \%$ higher than that at Point A. 


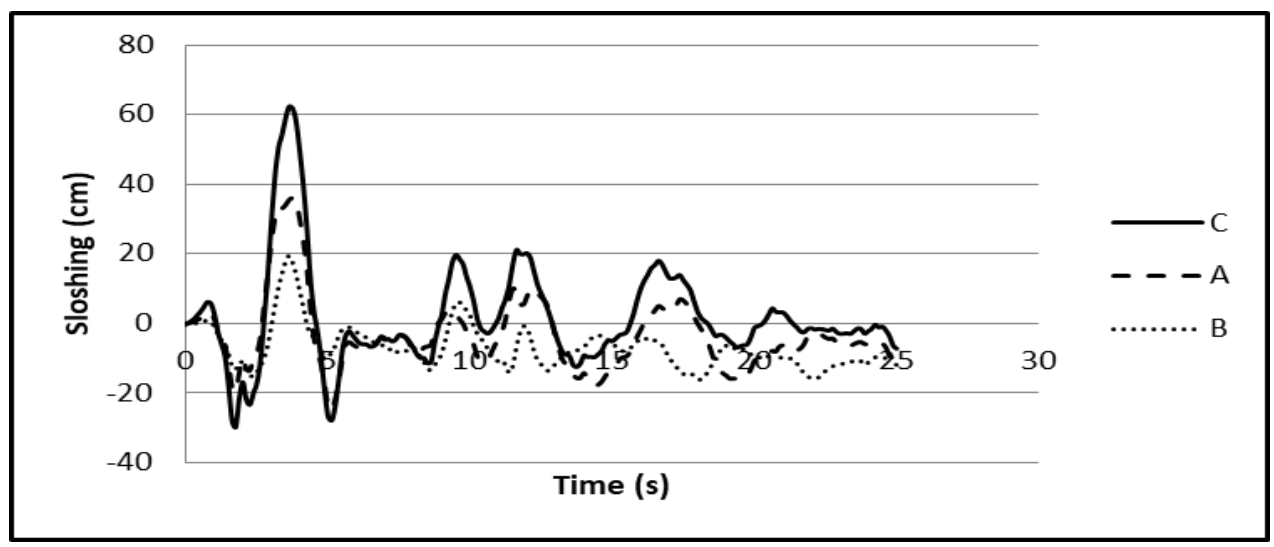

Figure 5.18: Time history of sloshing height for model "TX20Z10"

Figure 5.18 contains sloshing information for another tall tank case. Again for this model, the maximum sloshing happens at almost the same point in time as the previous model. The peak sloshing occurs 3.75 seconds after the excitation starts. The peak value of sloshing is increased by $58 \%$ from Point $\mathrm{A}$ to Point $\mathrm{C}$ as a result of the corner sloshing effect.

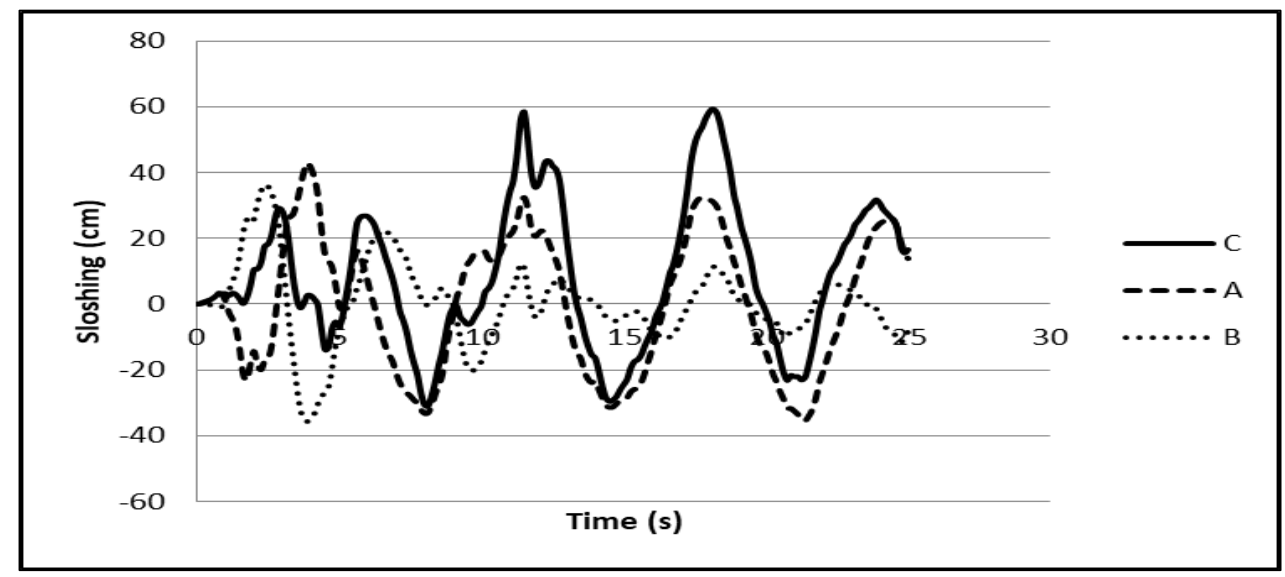

Figure 5.19: Time history of sloshing height for model "SX30Z15"

Model SX30Z15 can be categorized as a shallow tank. The water level for this model group is the deepest among all tanks in this study. As can be clearly observed in the figure, the sloshing graphs are well-separated for the three picked points. The sloshing response of the 
liquid particles located on the corner of the tank hits the maximum value twice at $\mathrm{t}=11.5 \mathrm{sec}$ and $\mathrm{t}=18 \mathrm{sec}$.

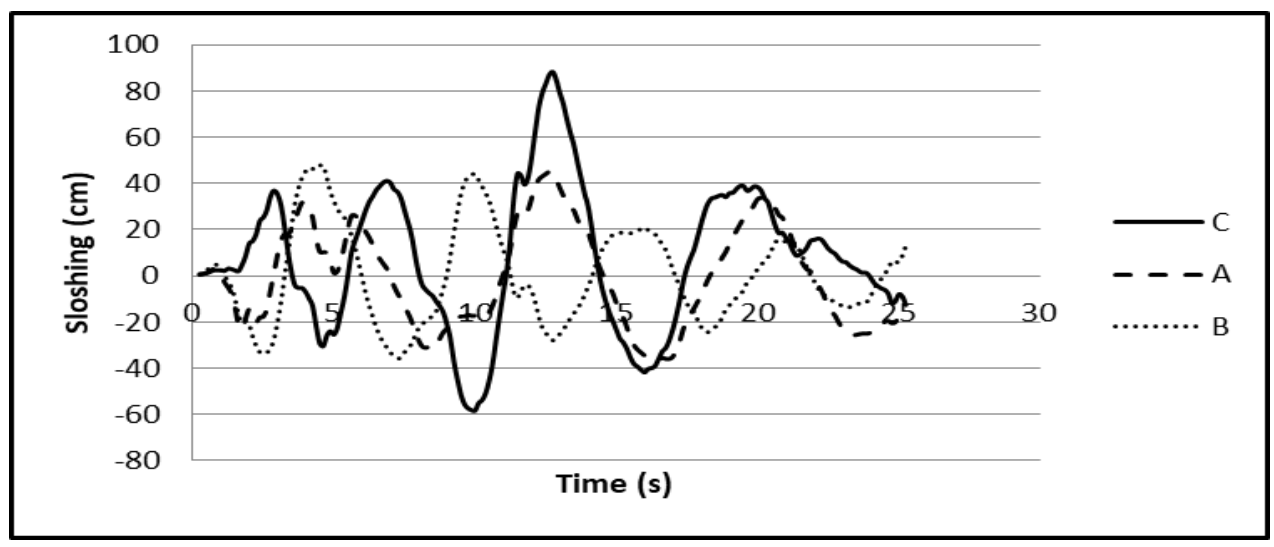

Figure 5.20: Time history of sloshing height for model "SX40Z20"

The trend that was mentioned about the SX30Z15 has gotten more obvious in the time history of sloshing in SX40Z20 and SX50Z25. According to the figure, peak sloshing values corresponding to the three selected points happen at different times during the analysis.

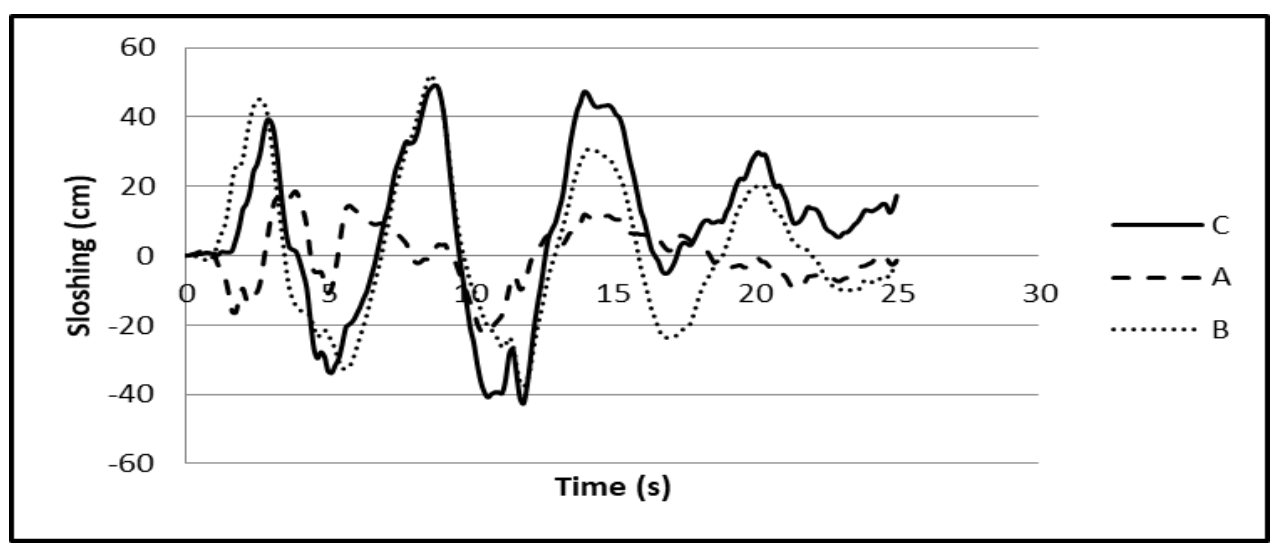

Figure 5.21: Time history of sloshing height for model "SX50Z25" 
Paying attention to the time history of sloshing for the first group of tanks reveals that for all models the maximum sloshing effect is mostly contributed by the critical component of the motion applied in the tank's critical direction. In fact, in all cases the sloshing at Point A is higher than that of Point B and its peak occurs closer in time to the corner's peak.

For the next group of tanks $\left(L_{x} / L_{z}=0.5\right)$, the El-Centro's components remain in the same directions as before. In fact, for this group the E-W component is applied in the critical direction of the tanks, which is $\mathrm{Z}$ axis now. The main purpose here is to study the comparative significance of the two important factors in controlling the sloshing behavior of the tanks namely; plan's aspect ratio and seismic amplitude.

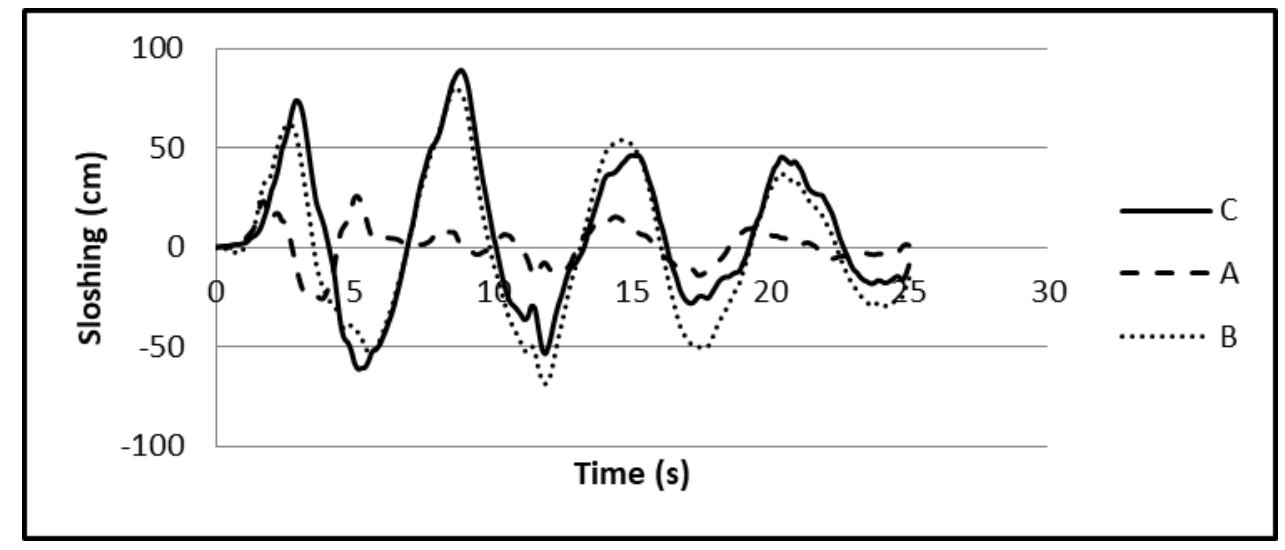

Figure 5.22: Time history of sloshing height for model "TX16Z32"

The first thing to be noticed in the figure is that the sloshing height at Point $\mathrm{B}$ is significantly higher than that of Point A. Moreover, by comparing the time history of sloshing between the two models TX16Z32 and TX16Z8, it becomes clear that a higher peak corner sloshing is experienced in model TX16Z32. 


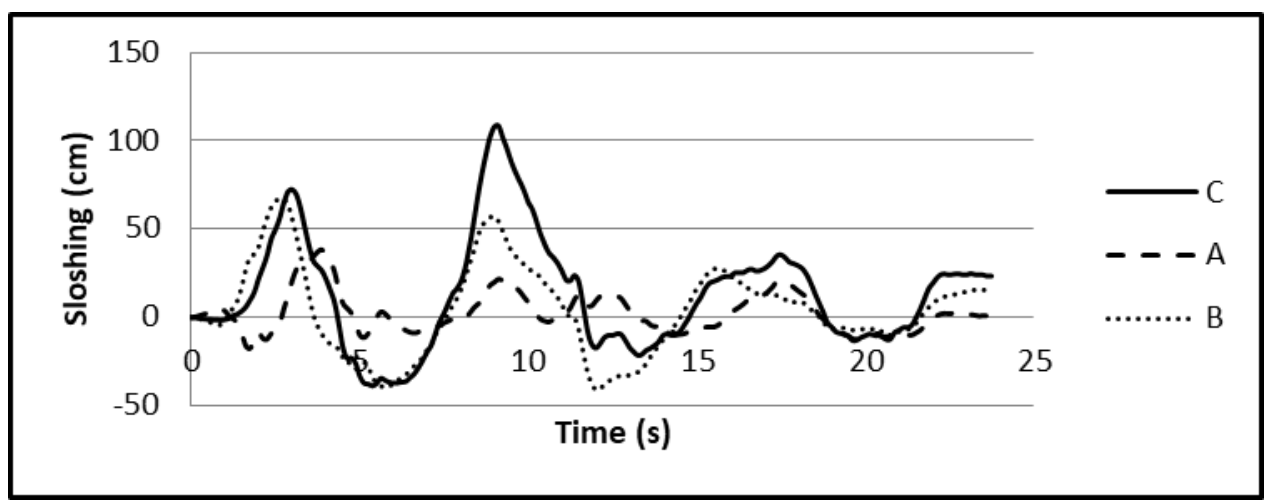

Figure 5.23: Time history of sloshing height for model "TX20Z40"

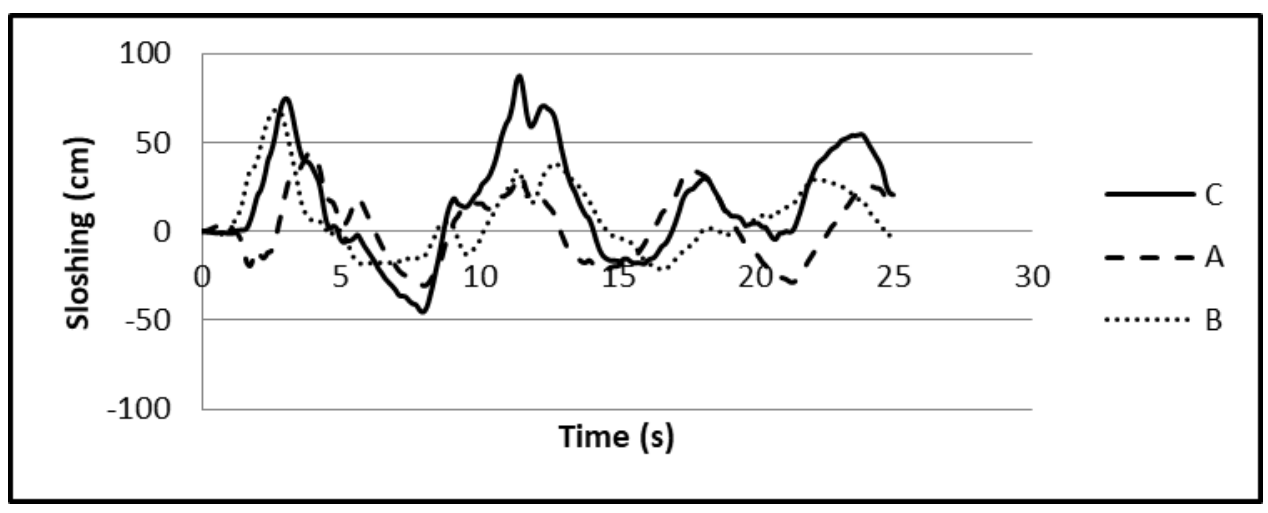

Figure 5.24: Time history of sloshing height for model "SX30Z60"

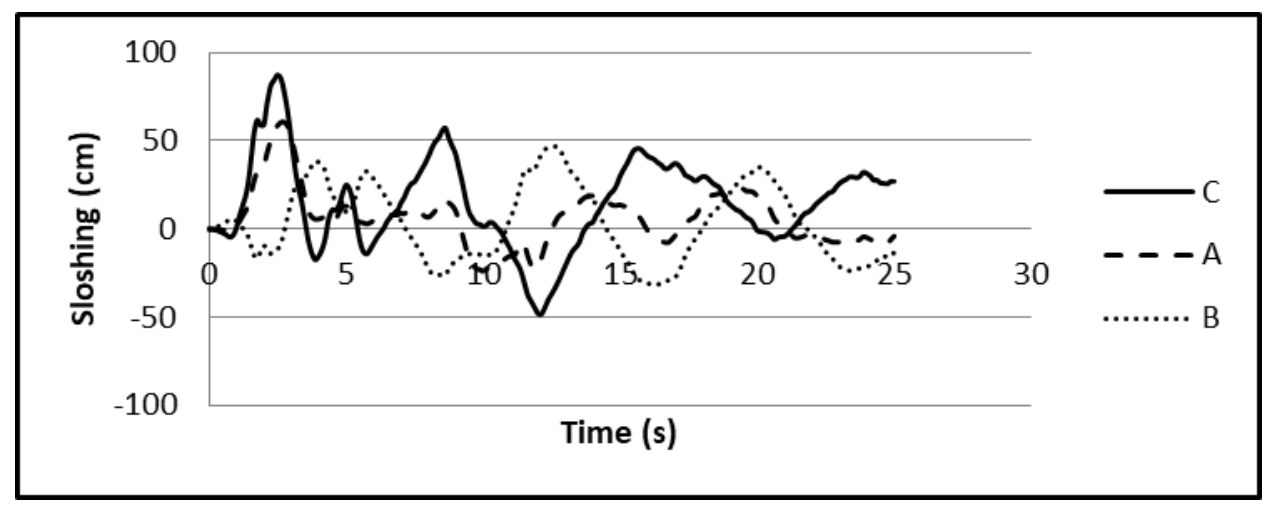

Figure 5.25: Time history of sloshing height for model "SX40Z80" 
The comparison between the calculated responses for the two groups of the tanks clarifies that the effect of the tank plan's aspect ratio on the sloshing properties is more significant than that of the motion's amplitude. For all models the time history of sloshing shows that the maximum sloshing height at Point B is getting closer to the maximum corner sloshing value.

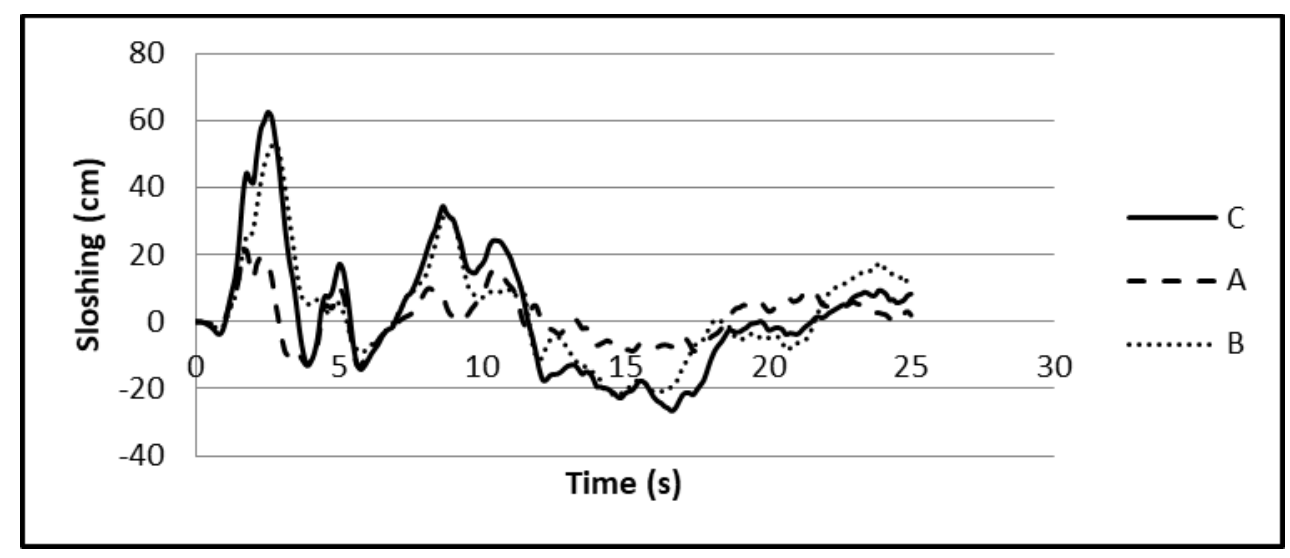

Figure 5.26: Time history of sloshing height for model "SX50Z100"

The current results can explain why in some past earthquakes the damage level on liquid tanks located at the same location were different. For example, Housner (1969) stated that during the earthquake of Chilean (1960) severe damages were reported on some liquid tanks while others experienced no damage. The provided results show how the sloshing performance of liquid tanks is sensitive to the variations of tanks' geometries. No change is made to the models of the two groups except for the length of the wall in $\mathrm{Z}$ direction; however, a considerable difference can be observed in the results estimated for the two groups.

Table 5.10 contains some detailed information on the value of peak sloshing height calculated by ACI 350.3-06 and the nonlinear time history FE analysis for the first group of the tanks with the plan aspect ratio of $\left(L_{x} / L_{z}=2\right)$ : 
Table 5.10: Maximum sloshing heights from ACI 350-3-06 and nonlinear FE analysis

\begin{tabular}{|c|c|c|c|c|}
\hline Types & Names & $d_{\max }(\mathrm{cm})$ & $\begin{array}{c}\text { NL-Sloshing } \\
(\mathrm{cm})\end{array}$ & $\begin{array}{c}\text { Insufficiency } \\
(\%)\end{array}$ \\
\hline \multirow{3}{*}{ Shallow } & SX50Z20 & 41.64 & 57.59 & 38.3 \\
\cline { 2 - 5 } & SX40Z20 & 69.69 & 88.24 & 26.6 \\
\cline { 2 - 5 } & SX30Z15 & 86.65 & 59.37 & - \\
\hline \multirow{2}{*}{ Tall } & TX20Z10 & 92.69 & 62.29 & - \\
\cline { 2 - 5 } & TX16Z8 & 93.99 & 62.29 & - \\
\hline
\end{tabular}

Comparing the results given in the table reveals that the code adequately overestimates the sloshing response of liquid in tall tanks. However, in shallow tanks the code has considerably underestimated the maximum liquid sloshing response. This insufficiency is getting more pronounced in wider tank. The insufficiency percentage for model SX40Z20 is $27 \%$ while for the wider tank SX50Z20 the percentage reaches 38\%. The code underestimation gets more obvious as the sloshing period increases. Moreover, the code inadequacy in estimating the sloshing response of the tanks is felt more acutely under the bidirectional seismic excitation case scenario as compared to the case of unidirectional loading.

Table 5.11 contains some detailed information on the value of peak sloshing height calculated by ACI 350.3-06 and the nonlinear time history FE analysis for the tanks with the plan aspect ratio of $\left(L_{x} / L_{z}=0.5\right)$ :

Table 5.11: Maximum sloshing heights from ACI 350-3-06 and nonlinear FE analysis

\begin{tabular}{|c|c|c|c|c|}
\hline Types & Names & $d_{\max }(\mathrm{cm})$ & $\begin{array}{c}\text { NL-Sloshing } \\
(\mathrm{cm})\end{array}$ & $\begin{array}{c}\text { Insufficiency } \\
(\%)\end{array}$ \\
\hline \multirow{3}{*}{ Shallow } & SX50Z100 & 41.64 & 69.73 & 67 \\
\cline { 2 - 5 } & SX40Z80 & 69.69 & 89.26 & 28 \\
\cline { 2 - 5 } & SX30Z60 & 86.65 & 82.26 & - \\
\hline \multirow{2}{*}{ Tall } & TX20Z40 & 92.69 & 108.42 & 17 \\
\cline { 2 - 5 } & TX16Z32 & 93.99 & 93.97 & - \\
\hline
\end{tabular}


It should be noted that the second group of the tanks are the wider cases of the tanks in the first group. Tanks in both groups have the same water levels, and the length of the walls in $\mathrm{X}$ direction is equal. The only difference is that the length of the walls in $\mathrm{Z}$ direction is 4 times longer than that of the first group. As can be seen from the table, the peak sloshing response is considerably underestimated by ACI 350.3-06 in three out of five cases considered. The obtained excessive insufficiency percentages could be translated as the direct outcome of making the tanks wider in this group. The response estimated for both groups of the tanks addresses the lack of accuracy of the approach adopted in the code in predicting the sloshing response of the wider tank models.

\subsubsection{Effect of Applying Bidirectional Seismic Component on Sloshing Response}

As mentioned before, ACI 350.3-06 computes minimum seismic design loads based on the single component of seismic ground motion. In fact, for calculating forces, stresses, displacements, and etc. for both impulsive and convective parts of hydrodynamic response, ACI standard considers the critical amplitude of earthquake applied in the critical direction of the tank (longer dimension in plan). The purpose of this subsection is to study the interaction effect of the second component of earthquake on the sloshing response of the contained liquid which is overlooked in ACI 350.3-06.

Table 5.12 provides the sloshing response values of the tanks with $\left(L_{x} / L_{z}=2\right)$ under both unidirectional and bidirectional excitation case scenarios:

Table 5.12: Effect of bidirectional seismic excitation on maximum sloshing height

\begin{tabular}{|c|c|c|c|c|c|}
\hline \multirow{3}{*}{ Types } & \multirow{3}{*}{ Names } & \multicolumn{4}{|c|}{ Sloshing Height (cm) } \\
\cline { 3 - 6 } & & Unidirectional & \multicolumn{2}{c|}{ Bidirectional } \\
\cline { 3 - 6 } & & $\mathrm{A}$ & $\mathrm{C}$ & $\mathrm{A}$ & $\mathrm{C}$ \\
\hline \multirow{3}{*}{ Shallow } & SX50Z20 & 21.31 & 28.31 & 19.51 & 57.59 \\
\cline { 2 - 6 } & SX40Z20 & 45.63 & 60.19 & 45.11 & 88.24 \\
\cline { 2 - 6 } & SX30Z15 & 39.41 & 47.49 & 39.37 & 59.37 \\
\hline \multirow{3}{*}{ Tall } & TX20Z10 & 32.42 & 43.57 & 39.37 & 62.29 \\
\cline { 3 - 6 } & TX16Z8 & 32.86 & 36.42 & 36.07 & 62.29 \\
\hline
\end{tabular}


In Table 5.13, the peak sloshing results of the tanks under bidirectional effect of ElCentro (1940) are normalized with respect to those of unidirectional case in order to provide a better understanding on the effect of the transverse seismic component. The values presented in Tables 5.12 and 5.13 could lead to several conclusions. First, nonlinear FE analysis agrees well with the ACI.350.3-06 approach ignoring the impact of the transverse component of ground motion on the sloshing response at the middle Point A. However, the FE analysis implies that the corner sloshing which is technically the maximum sloshing height in the tank to be considered for design purposes excessively increases as a result of applying the transverse component. Such effect is not adequately addressed by the ACI standard. Due to this bidirectional effect, the corner sloshing has increased from $43 \%$ to $71 \%$ in tall tanks while has increased from $25 \%$ to $100 \%$ in shallow tanks.

Table 5.13: Normalized sloshing heights

\begin{tabular}{|c|c|c|c|}
\hline Types & Names & A/A & C/C \\
\hline \multirow{3}{*}{ Shallow } & SX50Z20 & 0.92 & 2.03 \\
\cline { 2 - 4 } & SX40Z20 & 0.99 & 1.47 \\
\cline { 2 - 4 } & SX30Z15 & 1.00 & 1.25 \\
\hline \multirow{2}{*}{ Tall } & TX20Z10 & 1.21 & 1.43 \\
\cline { 2 - 4 } & TX16Z8 & 1.10 & 1.71 \\
\hline
\end{tabular}

It is quite obvious that the bidirectional effect could significantly intensify the sloshing response of the containers. ACI 350.3-06 does not account for such impact on the tanks' corner sloshing and completely overlooks this important effect in the estimation of hydrodynamic design loads and/or displacements. However, ASCE 7-05 proposes the Orthogonal Combination Procedure implying that such bidirectional interaction effect is deemed satisfied if the structure is designed in a three-dimensional space for 100 percent of the forces for one direction plus 30 percent of the forces for the perpendicular direction. It is observed that the maximum sloshing in the middle of the wall for any random direction is independent of the seismic component in the perpendicular direction. In order to investigate the sufficiency of the procedure suggested by ASCE 7-05, 100\% of the sloshing at Point A is combined with the $30 \%$ of the sloshing at Point B using the SRSS combination rule. According to the code, the combined results represent the corner sloshing which should be then compared with the FE results at Point $\mathrm{C}$ for verification 
purposes. Table 5.12 summarizes the estimated results using the two approaches. It is obvious from the table that ASCE 7-05 is also not capable of appropriately accounting for the effect of bidirectional excitation on the sloshing response. The proposed procedure of ASCE 7-05 underestimates the sloshing height from $8 \%$ in tall tanks to $29 \%$ in shallow tanks

Table 5.14: Orthogonality effect estimated by ASCE 7-05 and nonlinear FE analysis

\begin{tabular}{|c|c|c|c|c|}
\hline \multirow{2}{*}{ Types } & \multirow{2}{*}{ Names } & \multicolumn{2}{|c|}{ Max. Corner Sloshing (cm) } & \multirow{2}{*}{$\begin{array}{c}\text { Insufficiency } \\
\text { (\%) }\end{array}$} \\
\cline { 3 - 4 } & & ASCE 7-05 & Nonlinear FE & 29.27 \\
\hline \multirow{3}{*}{ Shallow } & SX50Z20 & 44.55 & 57.59 & 20.22 \\
\cline { 2 - 4 } & SX40Z20 & 73.40 & 88.24 & -5.95 \\
\cline { 2 - 4 } & SX30Z15 & 63.13 & 59.37 & 8.14 \\
\hline \multirow{2}{*}{ Tall } & TX20Z10 & 57.60 & 62.29 & 13.75 \\
\cline { 2 - 4 } & TX16Z8 & 54.76 & 62.29 & \\
\hline
\end{tabular}

\subsubsection{Effect of Earthquake Frequency Content}

As mentioned before, the sloshing performance of liquid tanks depends on different parameters such as; tank geometry, water level, frequency content and peak ground acceleration of earthquake. The main purpose of this section is to study the effect of earthquake frequency content on the nonlinear sloshing response of liquid storage tanks.

Three seismic records having different frequency content properties including El-Centro (1940), Northridge (1994) and San-Francisco (1957) are considered. The effect of both single directional and bidirectional excitation will be investigated. It should be noted that all records are scaled in such a way that the peak ground acceleration in the longitudinal direction becomes 0.4g. Figure 5.20 indicates the scaled time history records employed for this part.

The earthquake ground motions are generally classified with respect to the ratio of their peak ground acceleration (PGA) to peak ground velocity (PGV). If the ratio is more than 1.2, it is classified as high frequency, for the ratios between 1.2 and 0.8 , it is considered as intermediate and for all ratios less than 0.8 it is assumed as low frequency type. Therefore the Northridge would be classified as low frequency; El-Centro as intermediate frequency, and San-Francisco as 
high frequency. It should be noted that the predominant period of Northridge, El-Centro, and San-Francisco records are $0.708 \mathrm{sec}, 0.5 \mathrm{sec}$, and $0.22 \mathrm{sec}$, respectively.

In Table 5.13, the values of maximum sloshing at Points $\mathrm{C}$ and A located at the tank's corner and the middle of the wall perpendicular to the $\mathrm{N}-\mathrm{S}$ component of the records are presented. The listed results are for the case of unidirectional excitation.

Table 5.15: Peak FE sloshing heights under Northridge, El-Centro, and San-Francisco (unidirectional excitation)

\begin{tabular}{|c|c|c|c|c|c|c|c|c|}
\hline \multirow{3}{*}{ Types } & \multirow{3}{*}{ Groups } & \multirow{3}{*}{ Names } & \multicolumn{6}{|c|}{ Sloshing Height $(\mathrm{cm})$} \\
\hline & & & \multicolumn{2}{|c|}{ Northridge } & \multicolumn{2}{|c|}{ El-Centro } & \multicolumn{2}{|c|}{ San-Francisco } \\
\hline & & & A & $\mathrm{C}$ & $A$ & $\mathrm{C}$ & $A$ & $C$ \\
\hline \multirow{2}{*}{ Shallow } & Group 1 & SX40Z20 & 40.56 & 45.45 & 45.63 & 60.16 & 4.00 & 6.00 \\
\hline & Group 2 & SX30Z15 & 44.67 & 50.90 & 39.41 & 47.09 & 4.50 & 10.00 \\
\hline \multirow{2}{*}{ Tall } & Group 3 & TX20Z10 & 41.88 & 46.42 & 38.09 & 43.57 & 4.50 & 10.80 \\
\hline & Group 4 & TX16Z8 & 38.99 & 42.15 & 32.86 & 36.42 & 5.50 & 9.80 \\
\hline
\end{tabular}

Previous studies such as Kianoush and Ghaemmaghami (2011) have founded out that earthquake frequency content could have an important effect on the structural response of liquid storage tanks. All the records considered have the same peak ground acceleration of $0.4 \mathrm{~g}$ but as can be seen from the table the calculated time history results could be totally different from case to case. This clearly implies the importance of the effect of earthquake frequency content on the sloshing behavior of such structures.

In General, the sloshing response due to Northridge and El-Centro is significantly higher than that due to San-Francisco. This shows that low and intermediate frequency records could excessively intensify the convective component of response in such structures.

It is observed that for tall tanks the sloshing response under Northridge is higher than that under El-Centro while an opposite trend is found for shallow tanks. By considering the fundamental convective periods of the models, it can be concluded that for tanks with lower natural periods corresponding to the fundamental convective mode, higher sloshing height is caused by Northridge. On the other hand, for shallow tanks with longer fundamental sloshing 
periods, higher sloshing is resulted by El-Centro. This could be justified by the resonance effect due to the close vicinity of the fundamental sloshing period of the contained liquid with the predominant period of the record.

\subsubsection{Effect of Earthquake Frequency Content on Wave Interference}

In this sub-section, the impact of the frequency content of seismic ground motions on wave interference will be studied. In order to investigate the wave interference, the transverse components of the three seismic records presented in the previous part will be added to the simulation of the tanks. The N-S component of the records is applied in X direction, while the E$\mathrm{W}$ component is applied in $\mathrm{Z}$ direction.

The total sloshing height depends on the interaction of seismic waves in different directions. In some cases it is observed that under a unidirectional excitation one record could result in a higher sloshing response compared to the other one but a reverse trend is observed under a bidirectional motion for the same set of records. To study the subject the tanks are analyzed under the simultaneous action of the N-S and E-W components of Northridge (1994) and El-Centro (1940). The reason for choosing these records is that the sloshing response resulted by the two records was proved to be very close according to the results of the previous section, thus examining the combined response after adding the second component can produce interesting insight into the subject. The obtained maximum sloshing height values are given in Table 5.14:

Table 5.16: Maximum time history sloshing heights for El-Centro and Northridge

\begin{tabular}{|c|c|c|c|c|c|c|c|c|}
\hline \multirow{3}{*}{ Types } & \multirow{3}{*}{ Groups } & \multirow{3}{*}{ Names } & \multicolumn{6}{|c|}{ Sloshing Height $(\mathrm{cm})$} \\
\hline & & & \multicolumn{3}{|c|}{ Northridge } & \multicolumn{3}{|c|}{ El-Centro } \\
\hline & & & C & $A$ & B & C & $A$ & B \\
\hline \multirow{2}{*}{ Shallow } & Group 1 & SX40Z20 & 45.57 & 40.61 & 19.00 & 88.24 & 45.11 & 45.36 \\
\hline & Group 2 & SX30Z15 & 52.01 & 45.09 & 26.66 & 59.37 & 39.80 & 37.09 \\
\hline \multirow{2}{*}{ Tall } & Group 3 & TX20Z10 & 47.77 & 42.08 & 17.06 & 62.29 & 39.77 & 24.34 \\
\hline & Group 4 & TX16Z8 & 42.96 & 39.01 & 12.10 & 62.29 & 36.07 & 25.80 \\
\hline
\end{tabular}


A quick comparison between the maximum sloshing height in specified locations of the tanks for two earthquake records reveals that similar to the value of sloshing for single earthquake component, the values of liquid sloshing in point A for Northridge is higher than the value from El-Centro, it means that by ACI 350.6-06 approach by considering the critical seismic amplitude toward critical direction the sloshing performance of Northridge would be dominant. However, the simulation shows that by applying the E-W component of those seismic ground motions the resultant maximum sloshing heights in both point $\mathrm{C}$, and point be from ElCentro is significantly higher. Both the value of amplitude and the interaction between bidirectional waves can be the reason for the sloshing heights between two different ground motions. For further clarification, the sloshing heights from Table 5.14 are normalized with respect to the values from Northridge as presented in the following table:

Table 5.17: Relative maximum sloshing heights

\begin{tabular}{|c|c|c|c|c|c|}
\hline \multirow{2}{*}{ Types } & \multirow{2}{*}{ Groups } & \multirow{2}{*}{ Names } & \multicolumn{3}{|c|}{ Relative Sloshing } \\
\cline { 4 - 6 } & & & \multicolumn{2}{|c|}{ EL-Centro/Northridge } \\
\cline { 4 - 6 } & & & $\mathrm{C}$ & $\mathrm{A}$ & $\mathrm{B}$ \\
\hline \multirow{2}{*}{ Shallow } & Group 1 & SX40Z20 & 1.94 & 1.11 & 2.39 \\
\cline { 4 - 6 } & Group 2 & SX30Z15 & 1.14 & 0.88 & 1.39 \\
\hline \multirow{2}{*}{ Tall } & Group 3 & TX20Z10 & 1.30 & 0.95 & 1.43 \\
\cline { 4 - 6 } & Group 4 & TX16Z8 & 1.45 & 0.92 & 2.13 \\
\hline
\end{tabular}

The ratios of sloshing at point be shows that the E-W component of El-Centro is larger than the same component from Northridge, however, by paying attention to the ratio that shows the development of sloshing heights in points $\mathrm{C}$, it can be observed that the amplitude is not the only parameter that increased the sloshing heights in point C. For example, in the both tanks SX40Y20 and TX16Y8 the sloshing heights higher about $120 \%$ for El-Centro, but the resultant sloshing heights in points $\mathrm{C}$ is increased $45 \%$ in the TX16Y8, it is boosted $94 \%$ in the SX40Y20. Moreover, the sloshing heights enhanced about 40\% for both SX30Y15 and TX20Y10, but the maximum sloshing heights in points C improved 14\% and 30\% in SX30Y15 and TX20Y10. In conclusion, the higher maximum sloshing heights for El-Centro earthquake are not resulted 
directly for higher amplitude of ground motion in E-W direction, and to study the additional causes the time history liquid sloshing should be studied.

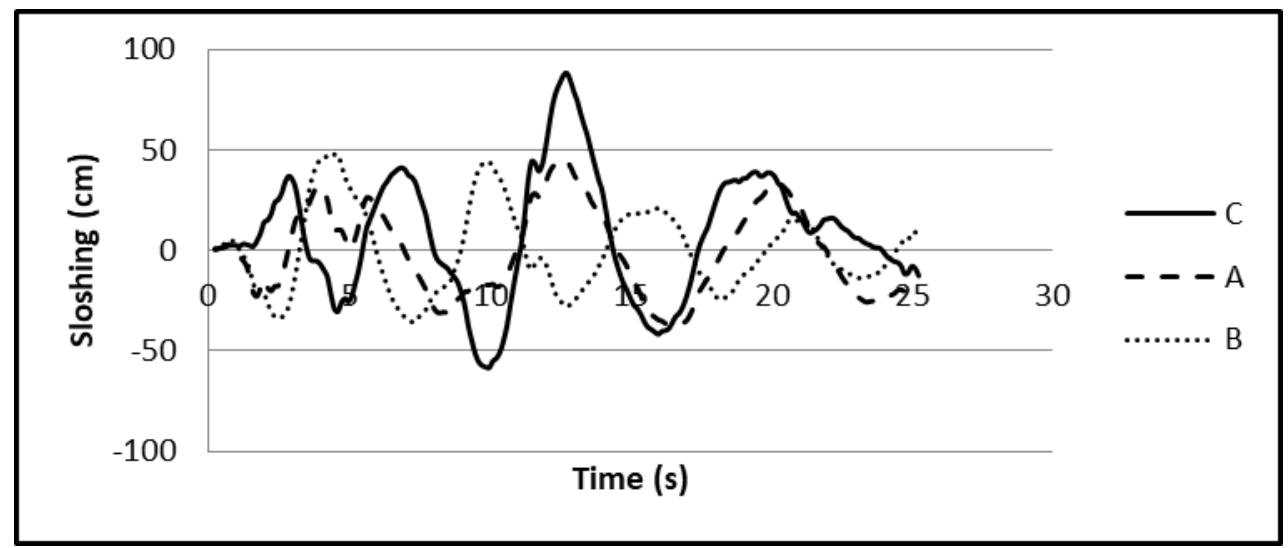

Figure 5.27: Time history of sloshing height for model "SX40Z20" under El-Centro

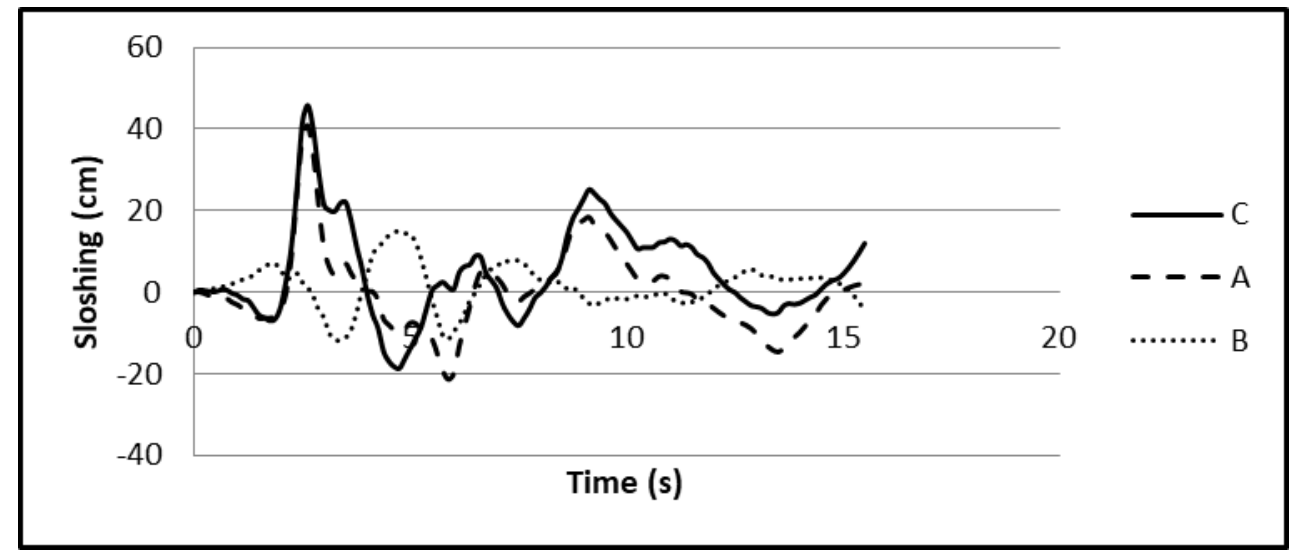

Figure 5.28: Time history of sloshing height for model "SX40Z20" under Northridge

Examining the obtained sloshing time history of the tank SX40Z20 shows that the main reason for the higher value of corner sloshing is simply because of the higher amplitude of seismic component in E-W direction. For both models the maximum corner sloshing happens almost at the same time that Point A reaches its maximum. Moreover, for both models the maximum sloshing of Point B occurs at different points in time; in fact, when the sloshing height 
values are maximum at Points $\mathrm{C}$ and $\mathrm{A}$, the value of sloshing at Point $\mathrm{B}$ is negative. It should be noted that SX40Z20 is the only case in which the sloshing height at Point A is higher for ElCentro among all different tank cases.

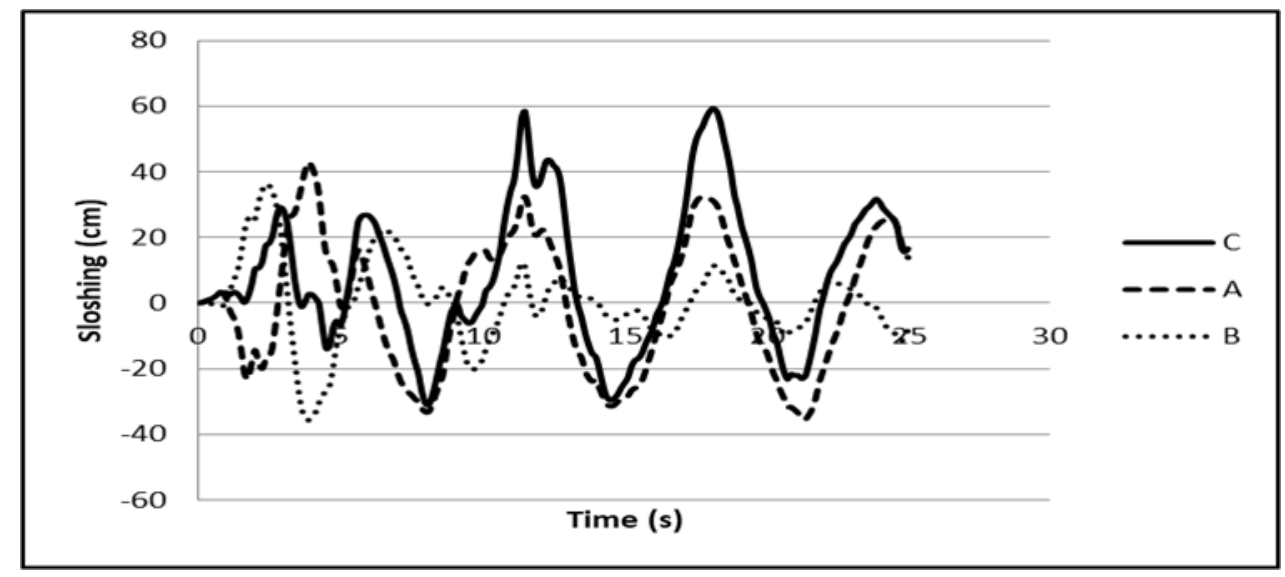

Figure 5:29: Time history of sloshing height for model "SX30Z15" under El-Centro

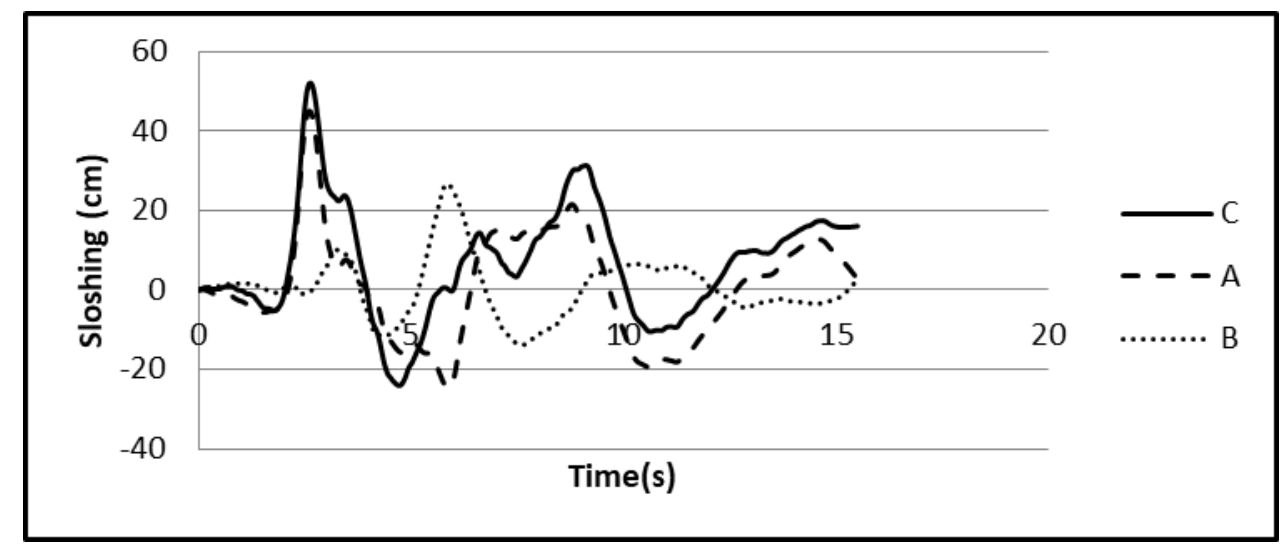

Figure 5.30: Time history of sloshing height for model "SX30Z15" under Northridge

Time history of sloshing for model SX30Z15 also shows that El-Centro has higher amplitude in $\mathrm{Z}$ direction of the tanks. Under El-Centro record the maximum sloshing at Point B happens 2.5 seconds after the beginning of the analysis. Moreover, when the sloshing height at Points $\mathrm{C}$ and $\mathrm{A}$ is maximum the sloshing value at Point $\mathrm{B}$ is positive meaning that the 
bidirectional waves are constructive. However, under Northridge record when the sloshing height at Points $\mathrm{C}$ and $\mathrm{A}$ is maximum, the sloshing height at $\mathrm{B}$ has not started to develop yet showing that the bidirectional waves act as non-constructive in this case.

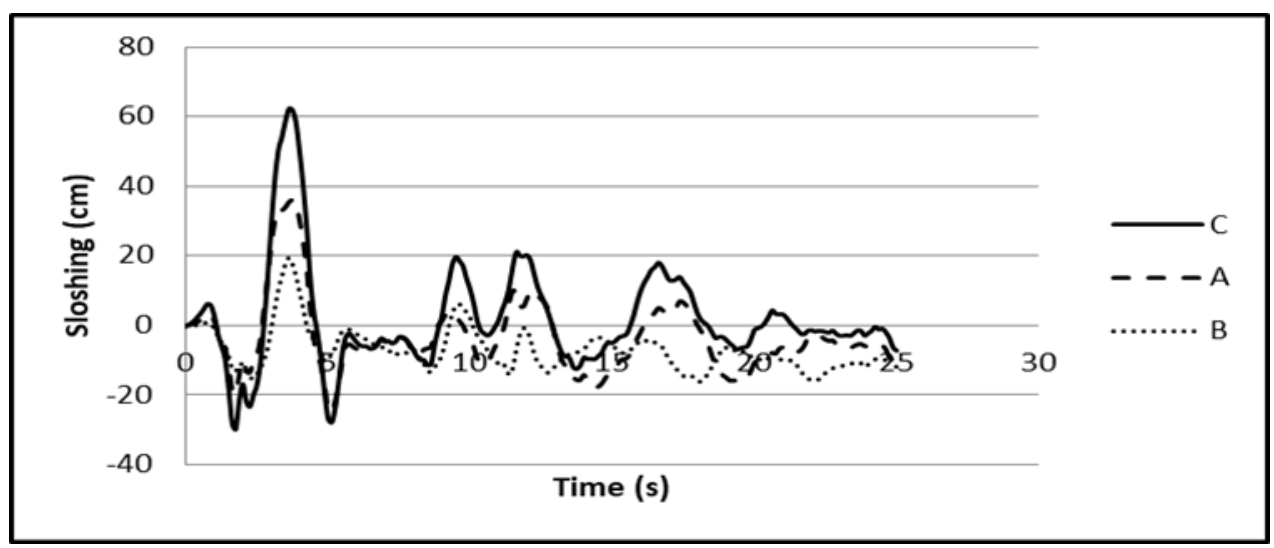

Figure 5.31: Time history of sloshing height for model "TX20Z10” under El-Centro

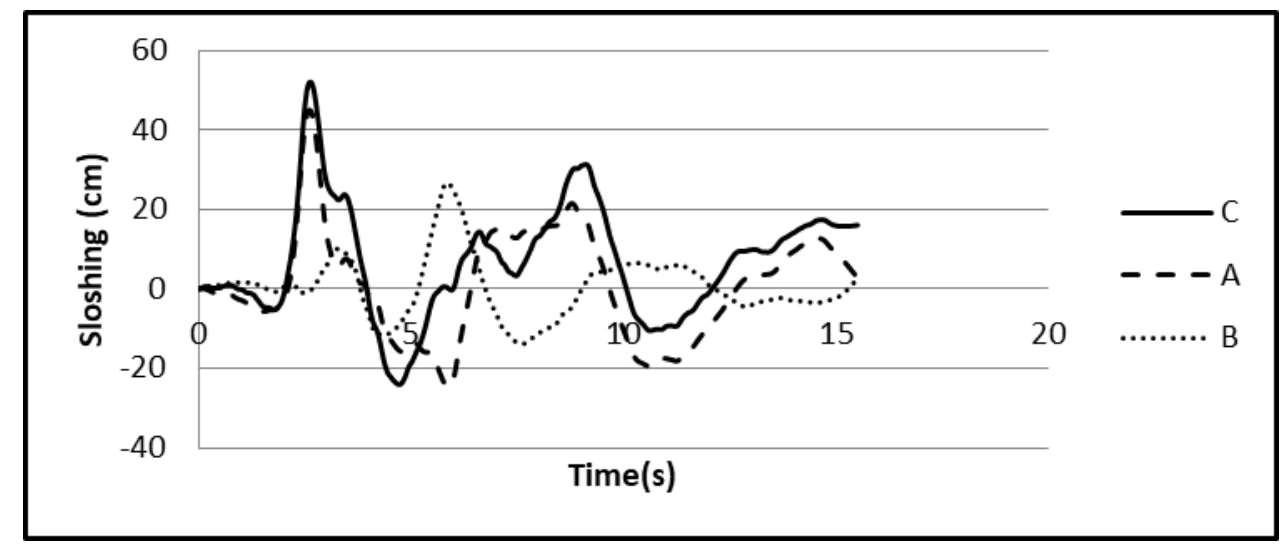

Figure 5.32: Time history of sloshing height for model "TX20Z10" under Northridge

The sloshing time history for model TX20Z10 under El-Centro record illustrates that the maximum of sloshing happens almost at the same time for all three points, indicating that the bidirectional waves are acting as constructive. On the other hand, under Northridge earthquake 
the sloshing at the corner is in phase with the sloshing at Point A whereas the sloshing response in $\mathrm{Z}$ direction is at the beginning stage and has not yet fully developed.

The percentage of increase in sloshing response for both models SX30Z15 and TX20Z10 at Point B resulted by the E-W components of the records is almost $40 \%$, however as a result of in-phase superposition of bidirectional waves at the corner of the tank, the amplitude of corner sloshing in model TX20Z10 is significantly increased by a factor of two compared to model SX30Z15.

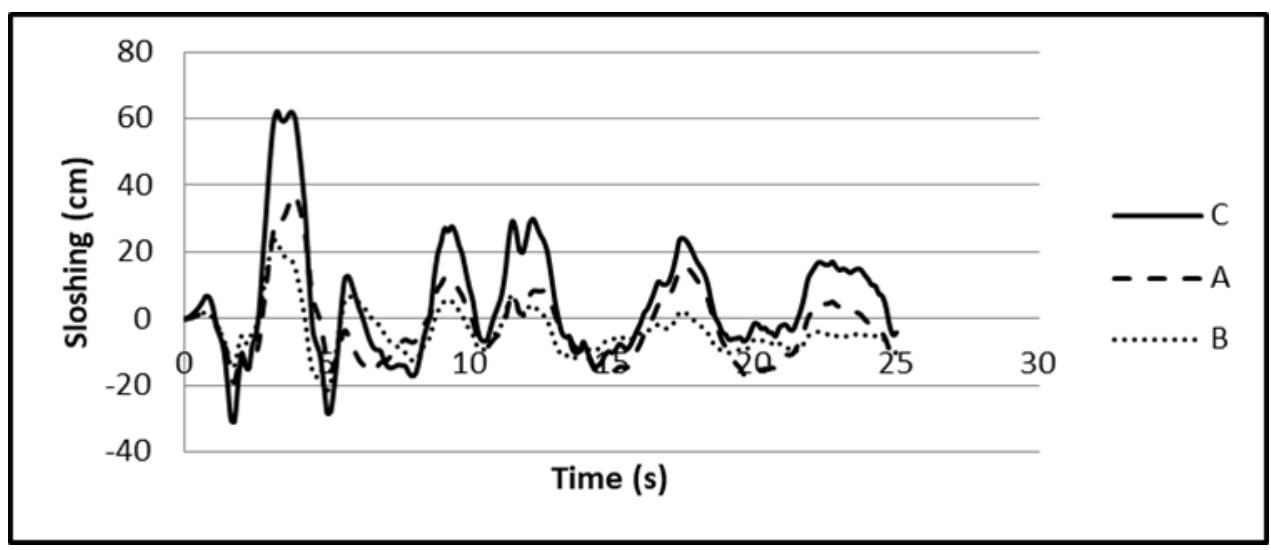

Figure 5.33: Time history of sloshing height for model "TX16Z8” under El-Centro

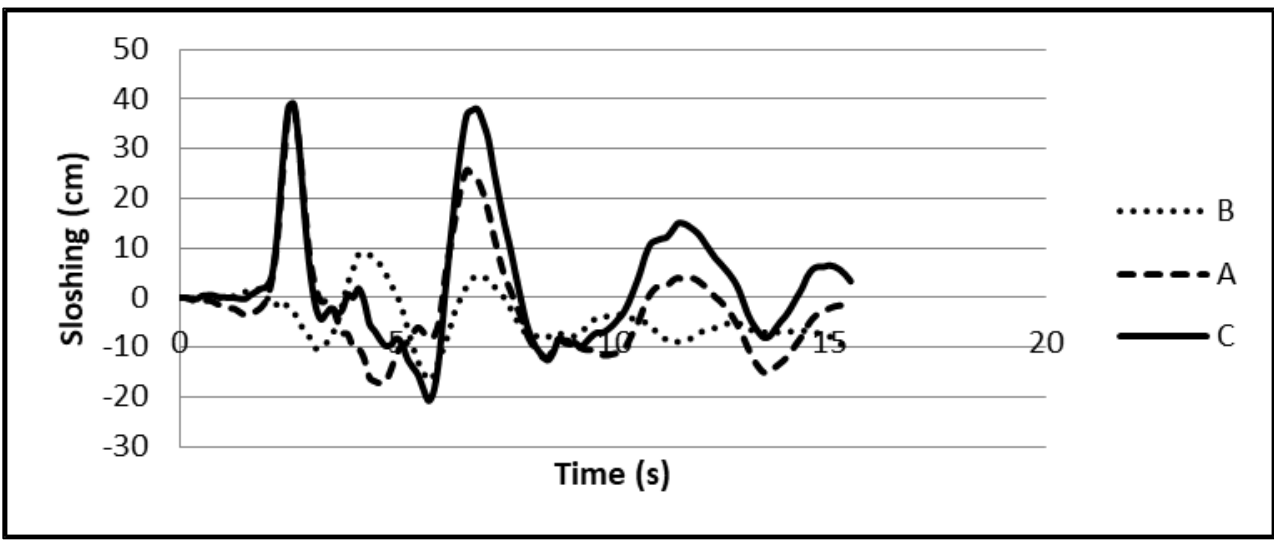

Figure 5.34: Time history of sloshing height for model "TX16Z8" under Northridge 
The sloshing time history for model TX16Z8 under El-Centro record illustrates that the maximum of sloshing happens almost at the same time at Points A and B. This has resulted in a significantly higher corner sloshing as compared to the case of Northridge record.

For better understanding of the interaction effect due to bidirectional waves, more attention is given to the sloshing time history response of model TX16Z8. The maximum sloshing of $40 \mathrm{~cm}$ has happened twice during the analysis; at $\mathrm{t}=2.5 \mathrm{sec}$ and $\mathrm{t}=6.75 \mathrm{sec}$. At $\mathrm{t}=$ $2.5 \mathrm{sec}$ the sloshing heights at Points $\mathrm{C}$ and $\mathrm{A}$ are almost equal while the sloshing value at Point $\mathrm{B}$ is almost zero. At $\mathrm{t}=6.75 \mathrm{sec}$ however the sloshing height at Point $\mathrm{A}$ is $22.05 \mathrm{~cm}$ and its value at Point $\mathrm{B}$ is $4 \mathrm{~cm}$. In fact, the equal corner sloshing height at the 6.75 seconds shows that even with weaker waves in $\mathrm{X}$ and $\mathrm{Z}$ directions, the constructive bidirectional wave effect has been able to produce the same amount of corner sloshing.

\subsubsection{Nonlinear Hydrodynamic Pressure on the Walls}

Hydrodynamic pressures acting on the walls of liquid storage tanks will be presented in this subsection. It should be noted that ABAQUS is only capable of calculating the total hydrodynamic forces or pressures. To validate the calculated FE pressures, the analytical models by Haroun (1983) and Housner (1968) are used for impulsive and convective components of response, respectively. To do so, the impulsive and convective parts are combined using the SRSS combination rule.

The hydrodynamic pressure is reported at the nodes on the middle section of the shorter wall perpendicular to the seismic component applied along X direction. It should be noted that in ABAQUS the surrounding walls are modeled as rigid body meaning that they act as completely rigid objects during the analysis. As a result no flexibility is associated with their performance.

In the following, some tables and figures are provided to provide more insight into the subject through comparison of the analytical linear pressures with the nonlinear FE estimations.

It should be noted that for this part of the study as the adopted analytical approach for calculating the hydrodynamic pressure response, Haroun (1984) and Housner (1963) formulations are used for estimating the impulsive and connective components, respectively. Most of the codes and standards around the world have adopted these two formulations as the basis for the hydrodynamic response analysis of the liquid-filled containers. The listed FE 
pressures are the maximum pressures at different levels along the height of the tank wall. The results represent the contact pressure at the interface between the liquid and structure domains from which the hydrostatic effect is taken out.

Table 5.16 summarizes the results obtained by the two approaches for model SX40Y20. For further clarification, Figure 5.26 is also provided to illustrate the distribution of seismically induced pressure along the height of the tank wall.

Table 5.18: Maximum hydrodynamic pressure in model "SX40Z20"

\begin{tabular}{|c|c|c|c|c|c|}
\hline \multirow{2}{*}{ Height $(\mathrm{m})$} & \multicolumn{4}{|c|}{ Hydrodynamic Pressure $(\mathrm{kPa})$} & \multirow{2}{*}{ Ratio } \\
\cline { 2 - 5 } & Convective & Impulsive & LI-Total & NL-Total & \\
\hline 0.00 & 1.21 & 32.38 & 32.40 & 31.27 & 0.97 \\
\hline 1.00 & 1.60 & 32.20 & 32.24 & 33.51 & 1.04 \\
\hline 2.00 & 1.99 & 31.76 & 31.82 & 33.66 & 1.06 \\
\hline 3.00 & 2.38 & 31.01 & 31.10 & 32.46 & 1.04 \\
\hline 4.00 & 2.77 & 29.89 & 30.02 & 31.37 & 1.04 \\
\hline 5.00 & 3.16 & 28.49 & 28.66 & 30.07 & 1.05 \\
\hline 6.00 & 3.55 & 26.65 & 26.88 & 28.18 & 1.05 \\
\hline 7.00 & 3.94 & 24.38 & 24.70 & 26.38 & 1.07 \\
\hline 8.00 & 4.33 & 21.69 & 22.12 & 24.28 & 1.10 \\
\hline 9.00 & 4.72 & 18.25 & 18.85 & 21.34 & 1.13 \\
\hline 10.00 & 5.11 & 14.10 & 15.00 & 17.89 & 1.19 \\
\hline 11.00 & 5.50 & 8.82 & 10.39 & 14.10 & 1.36 \\
\hline 12.00 & 5.89 & 0.06 & 5.89 & 9.30 & 1.57 \\
\hline
\end{tabular}




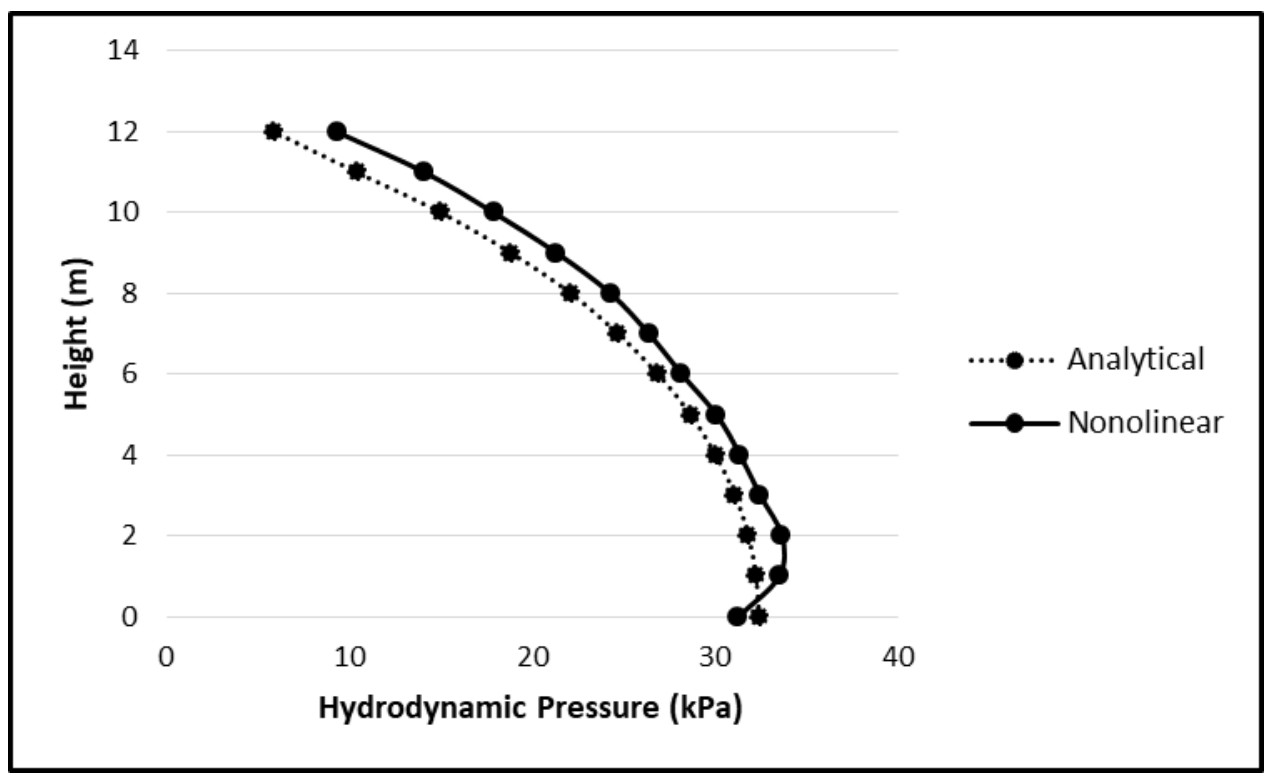

Figure 5.35: Comparison of linear analytical and nonlinear FE approach in model "SX40Z20"

The general conclusion to be drawn based on the table and the figure is that the linear approach can cover the hydrodynamic pressure for the impulsive part. In the last column of Table 5.16 the FE pressures are normalized with respect to the analytical values. It can be observed that in general the nonlinear FE approach has led to an overestimation of results, however the ratio constantly gets higher by moving toward the upper sections of the wall. Within the first two-third of the height measured from the base of the tank this difference is between $4 \%$ and 10\%; however, within the remaining height of the wall the difference ranges more considerably from $13 \%$ to $57 \%$. Considering the fact that the convective component usually dominates the hydrodynamic response in the upper portions of the wall, it can be concluded that linear formulation has underestimated the pressure for the convective part.

Table 5.19 presents the hydrodynamic pressure acting on the walls of model SX30Y15. It can be seen that nonlinear pressures are larger than the linear ones at all the measuring points along the height. The accuracy of the linear approach is getting lower on the upper part of the tank's wall. Both approaches estimate that the maximum hydrodynamic pressure happens at the base of the tank with the nonlinear response being $12 \%$ higher than the linear one. It was observed in the previous section that the maximum sloshing on the liquid free surface was 
underestimated by $67 \%$ by ACI 350.3-06 in comparison to the nonlinear FE approach for SX30Y15 under bidirectional El-Centro record. The pressure values reported here agree well with the previous observations by showing once again that the linear approach has underestimated the pressure at the top of the wall by $92 \%$. The inaccuracy observed in the calculated convective response by the linear approach could lead to an underestimation of the pressures at the upper parts of the tanks as well as an insufficient estimation of the tanks' freeboards.

Table 5.19: Maximum hydrodynamic pressure in model "SX30Z15"

\begin{tabular}{|c|c|c|c|c|c|}
\hline \multirow{2}{*}{ Height $(\mathrm{m})$} & \multicolumn{4}{|c|}{ Hydrodynamic Pressure $(\mathrm{kPa})$} & \multirow{2}{*}{ Ratio } \\
\cline { 2 - 5 } & Convective & Impulsive & LI-Total & NL-Total & \\
\hline 0.00 & 1.65 & 37.25 & 37.29 & 39.35 & 1.06 \\
\hline 1.00 & 1.86 & 37.10 & 37.15 & 41.16 & 1.11 \\
\hline 2.00 & 2.08 & 36.83 & 36.89 & 40.59 & 1.10 \\
\hline 3.00 & 2.30 & 36.27 & 36.34 & 39.55 & 1.09 \\
\hline 4.00 & 2.52 & 35.58 & 35.67 & 38.84 & 1.09 \\
\hline 5.00 & 2.74 & 34.56 & 34.67 & 37.80 & 1.09 \\
\hline 6.00 & 2.96 & 33.40 & 33.53 & 36.36 & 1.08 \\
\hline 7.00 & 3.18 & 31.85 & 32.01 & 34.86 & 1.09 \\
\hline 8.00 & 3.40 & 30.13 & 30.32 & 33.43 & 1.10 \\
\hline 9.00 & 3.62 & 27.92 & 28.15 & 32.20 & 1.14 \\
\hline 10.00 & 3.84 & 25.49 & 25.78 & 29.35 & 1.14 \\
\hline 11.00 & 4.06 & 22.39 & 22.75 & 26.11 & 1.15 \\
\hline 12.00 & 4.28 & 18.94 & 19.42 & 23.07 & 1.19 \\
\hline 13.00 & 4.50 & 14.39 & 15.08 & 18.88 & 1.25 \\
\hline 14.00 & 4.72 & 9.08 & 10.23 & 14.94 & 1.46 \\
\hline 15.00 & 4.94 & 0.00 & 4.94 & 9.50 & 1.93 \\
\hline
\end{tabular}




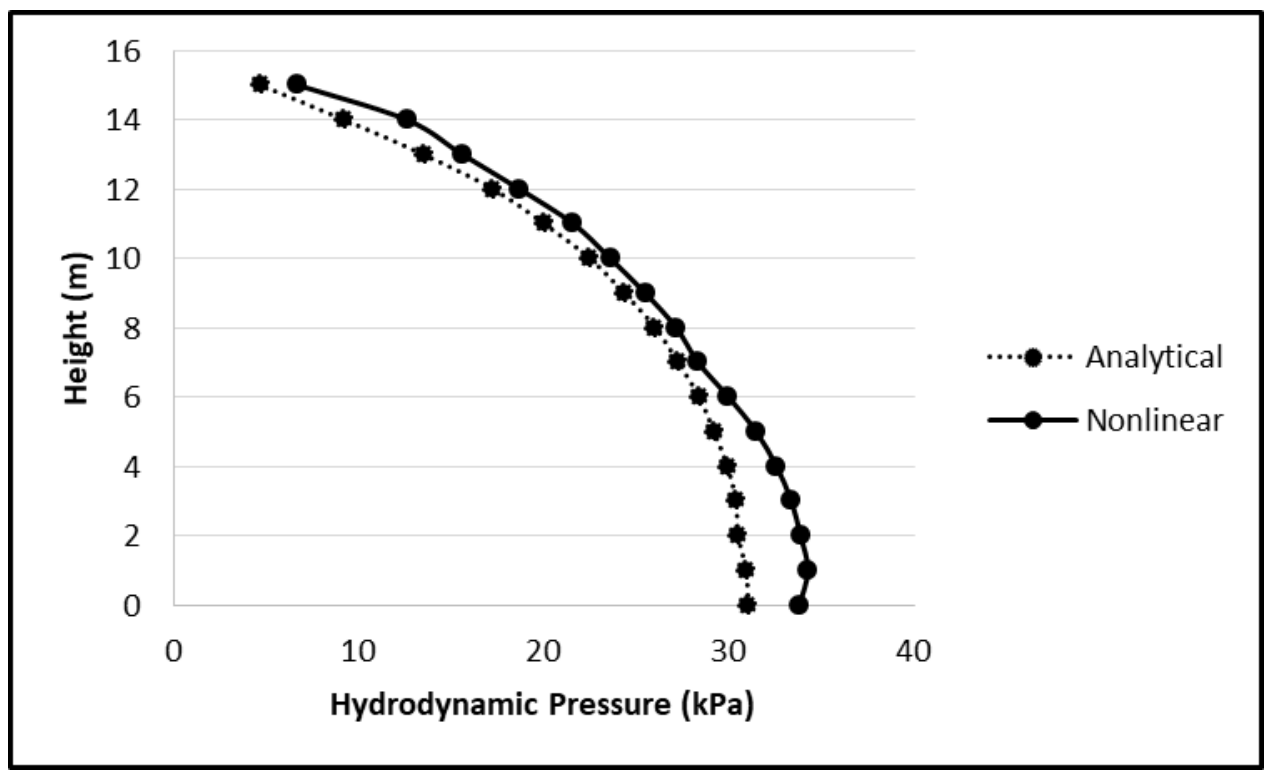

Figure 5.36: Comparison of linear analytical and nonlinear FE approach in model "SX30Z15"

Table 5.20 shows the maximum hydrodynamic pressures acting on the wall of the tank TX20Z10. Within the first two-third of the height measured from the base of the tank the linear approach has been able to predict a reasonable estimation of the hydrodynamic pressure with the difference being less than 14\%; however, within the remaining height of the wall the difference ranges more significantly from $15 \%$ to $93 \%$. As per the results of the previous section, a $17 \%$ insufficiency was observed in the freeboard calculations of the model using the linear approach which could be well justified with the results presented here for the hydrodynamic pressure. 
Table 5.20: Maximum hydrodynamic pressure in model "TX20Z10"

\begin{tabular}{|c|c|c|c|c|c|}
\hline \multirow{2}{*}{ Height $(\mathrm{m})$} & \multicolumn{4}{|c|}{ Hydrodynamic Pressure $(\mathrm{kPa})$} & \multirow{2}{*}{ Ratio } \\
\cline { 2 - 5 } & Convective & Impulsive & LI-Total & NL-Total & \\
\hline 0.00 & 0.33 & 31.02 & 31.02 & 33.79 & 1.09 \\
\hline 1.00 & 0.62 & 30.90 & 30.91 & 34.24 & 1.11 \\
\hline 2.00 & 0.91 & 30.47 & 30.48 & 33.85 & 1.11 \\
\hline 3.00 & 1.20 & 30.33 & 30.35 & 32.75 & 1.08 \\
\hline 4.00 & 1.49 & 29.87 & 29.91 & 32.26 & 1.08 \\
\hline 5.00 & 1.78 & 29.12 & 29.17 & 31.46 & 1.08 \\
\hline 6.00 & 2.07 & 28.30 & 28.38 & 28.16 & 0.99 \\
\hline 7.00 & 2.36 & 27.14 & 27.24 & 28.42 & 1.04 \\
\hline 8.00 & 2.66 & 25.87 & 26.01 & 26.34 & 1.01 \\
\hline 9.00 & 2.95 & 24.15 & 24.33 & 25.48 & 1.05 \\
\hline 10.00 & 3.24 & 22.24 & 22.47 & 23.08 & 1.03 \\
\hline 11.00 & 3.53 & 19.70 & 20.01 & 22.58 & 1.13 \\
\hline 12.00 & 3.82 & 16.82 & 17.25 & 17.59 & 1.02 \\
\hline 13.00 & 4.11 & 12.86 & 13.50 & 15.59 & 1.15 \\
\hline 14.00 & 4.40 & 8.10 & 9.22 & 12.60 & 1.37 \\
\hline 15.00 & 4.69 & 0.00 & 4.69 & 6.72 & 1.43 \\
\hline
\end{tabular}

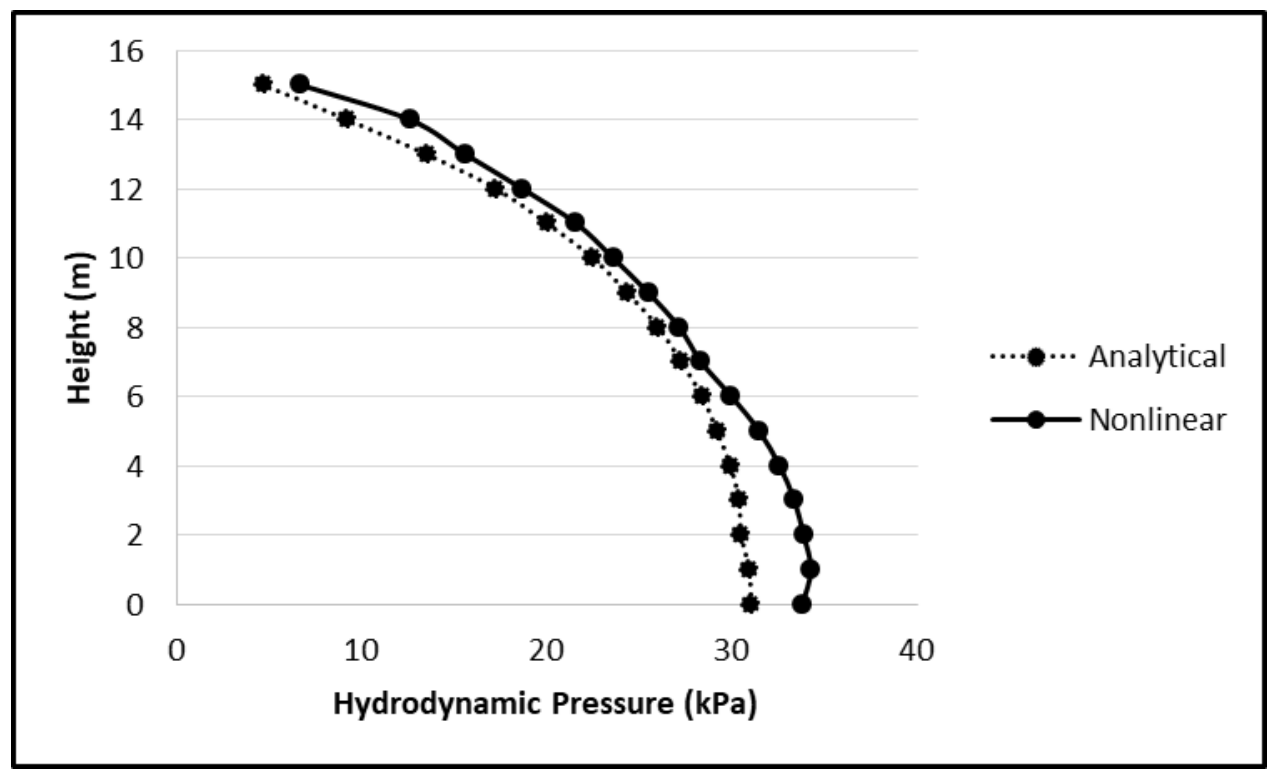

Figure 5.37: Comparison of linear analytical and nonlinear FE approach in model "TX20Z10" 
Table 5.21: Maximum hydrodynamic pressure in model "TX16Z8"

\begin{tabular}{|c|c|c|c|c|c|}
\hline \multirow{2}{*}{ Height $(\mathrm{m})$} & \multicolumn{4}{|c|}{ Hydrodynamic Pressure (kPa) } & \multirow{2}{*}{ Ratio } \\
\cline { 2 - 5 } & Convective & Impulsive & LI-Total & NL-Total & \\
\hline 1.00 & 0.00 & 29.09 & 29.09 & 29.35 & 1.01 \\
\hline 2.00 & 0.11 & 29.01 & 29.01 & 30.16 & 1.04 \\
\hline 3.00 & 0.42 & 28.74 & 28.74 & 30.47 & 1.06 \\
\hline 4.00 & 0.72 & 28.47 & 28.48 & 29.28 & 1.03 \\
\hline 5.00 & 1.02 & 27.99 & 28.01 & 28.34 & 1.01 \\
\hline 6.00 & 1.33 & 27.46 & 27.49 & 27.75 & 1.01 \\
\hline 7.00 & 1.63 & 26.72 & 26.77 & 27.21 & 1.02 \\
\hline 8.00 & 1.93 & 25.83 & 25.90 & 26.42 & 1.02 \\
\hline 9.00 & 2.24 & 24.71 & 24.81 & 25.08 & 1.01 \\
\hline 10.00 & 2.54 & 23.30 & 23.44 & 24.54 & 1.05 \\
\hline 11.00 & 2.85 & 21.60 & 21.79 & 21.05 & 0.97 \\
\hline 12.00 & 3.15 & 19.36 & 19.61 & 20.56 & 1.05 \\
\hline 13.00 & 3.45 & 16.73 & 17.08 & 18.49 & 1.08 \\
\hline 14.00 & 3.76 & 13.02 & 13.55 & 15.13 & 1.12 \\
\hline 15.00 & 4.06 & 8.50 & 9.42 & 11.19 & 1.19 \\
\hline 16.00 & 4.36 & 0.70 & 4.42 & 6.25 & 1.41 \\
\hline
\end{tabular}

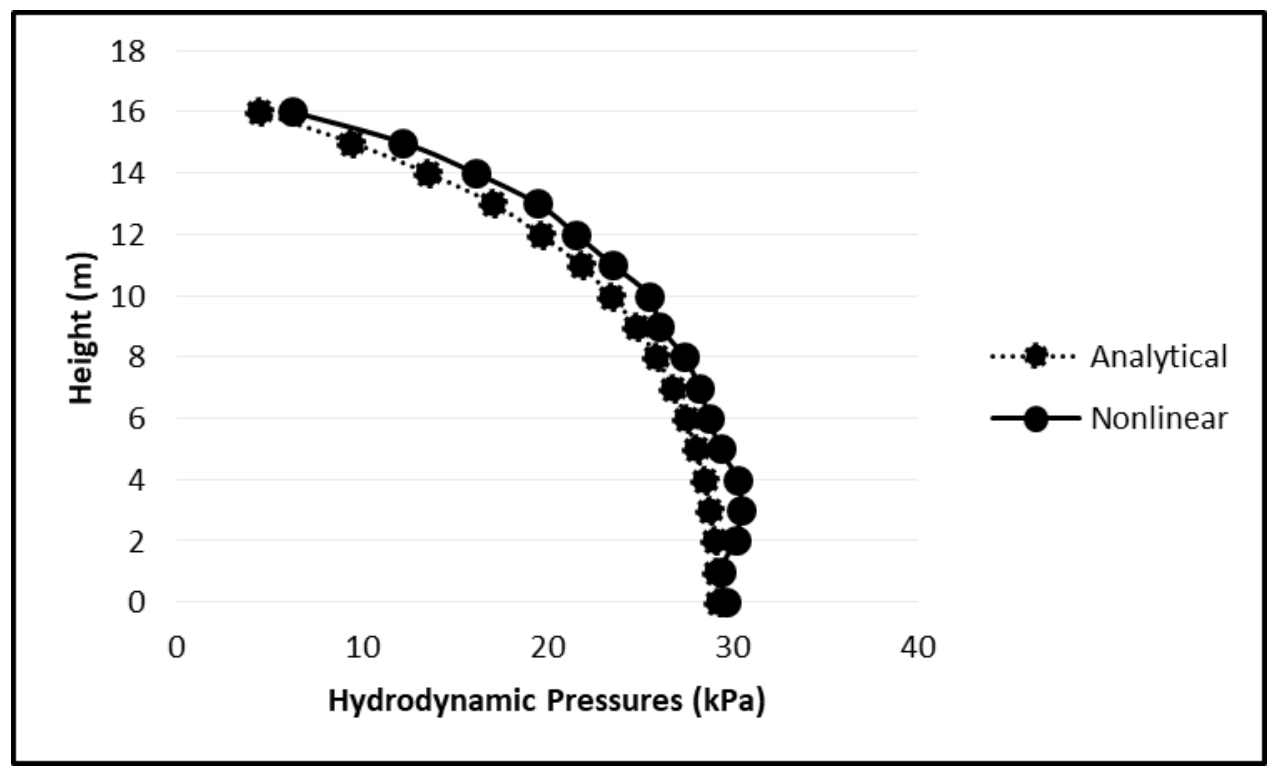

Figure 5.38: Comparison of linear analytical and nonlinear FE approach in model "TX16Z8" 
For the last tank studied in this section "TX16Y8", the linear methodology has estimated the pressure along the height of the wall with less than $11 \%$ inaccuracy for the first two-third of the height from the base. However, for the upper sections of the tank this difference varies more noticeably from $13 \%$ at the height of $11 \mathrm{~m}$ from the base to $43 \%$ on the free surface.

To sum up, the linear approach has shown enough sufficiency in estimating the impulsive component of hydrodynamic pressure, and has also yielded to more precise hydrodynamic behavior analysis of the tanks for tall configurations as compared to shallow ones. Furthermore, the linear theory reveals a significant lack of accuracy for hydrodynamic pressure estimation at upper height levels of the contained liquid.

Most of the previous studies available on the analytical solution for hydrodynamic pressure in liquid tanks imply that the amount of convective pressure is quite negligible in comparison with the impulsive part and as a result only little effort has been done to better identify this component analytically. The only analytical solution available for convective pressure is the Housner's formulation (1963) which is used as the basis for all the analytical calculations in this section. Moreover, all pressure response values provided in this section are reported at the middle sections of the walls for all considered models in order to make it possible to compare the obtained FE results with the linear analytical estimations.

It is important to note that according to the results of the previous section of this work, a higher convective pressure is expected at the corners of the tanks, indicating even a higher level of inadequacy of ACI linear formulation in predicting the sloshing response at critical corner points. This could be translated as a possible chance of vulnerability of liquid containers to excessive damage at the upper sections of the surrounding walls as well as the roof mounted on top. 


\section{Chapter 6}

\section{Summary, Conclusion, and Future Study}

\subsection{Summary}

Hydrodynamic response of liquid storage tanks has been the subject of many studies in the past. Each of these researches focused on a number of parameters which were found to be significant on performance of excited liquid containing tanks. However, poor performance of those structures based on damages reported from previous major earthquake reveals that there are many other important factors needed to be considered in design of liquid storage tanks. For improving the design approach of these structures the newly developed methodologies must maintain the features which simulate the real situations. For example, the effect of nonlinearity always exists in all conditions. To sum up, the major purpose of this study is to perform a nonlinear analysis on externally excited rectangular tanks by an earthquake ground motion.

Introducing a finite element 3D rectangular liquid storage tank model in maintaining nonlinearity is the most important purpose of the present work. Studying the nonlinear surface liquid sloshing performance and nonlinear hydrodynamic pressure acting on the tank walls under earthquake loading are set to be the first priority of this study.

In addition, some of important factors that affect the liquid sloshing performance are studied such as, frequency content of earthquake ground motion, tank plan dimension, and liquid level. In each part of this thesis nonlinear results are compared with the ACI 350.3-06 to evaluate the accuracy of the code.

A parametric study has been conducted to study effective parameters mentioned above. Twenty different rectangular tank configurations which are used mostly in industrial plants are simulated by nonlinear analysis using the ABAQUS/CAE (Version 2016) software program. The wall thickness of the simulated tanks is constant along the vertical height, and the structures are fixed at the base.

In previous studies conducted on this problem, a number of parameters have shown to have no or negligible effect on the sloshing performance of liquid storage tanks. For example, 
the vertical component of earthquake load has negligible effect on convective part of liquid domain. Moreover, flexibility of the wall has no effect on the sloshing height of the liquid. Based on conclusions from these studies, the effect of these parameters is ignored in this study.

It should be noted that the El-Centro (1940) earthquake is applied for the time history analysis in this study. The horizontal component (transversal and longitudinal) of this major seismic ground motion is used for nonlinear analysis of the liquid tanks. In this study, the N-S component of the El-Centro is scaled in way that its peak ground acceleration (PGA) becomes equal to $0.4 \mathrm{~g}$. Moreover, to study the frequency content of earthquake on wave interference in nonlinear condition four tank models are subjected to three different earthquake records. Northridge (1994), El-Centro (1940) and San-Francisco (1957) are the earthquake records used in this study and all of their peak ground acceleration scaled to $0.4 \mathrm{~g}$ in order to maintain different records with equal PGA.

\subsection{Conclusions}

- The nonlinear analysis shows that vertical distribution of hydrodynamic pressure acting on the wall of liquid storage tanks has no important difference with the calculated pressure based in linear elastic methodology. However, nonlinear hydrodynamic pressure values on the top of wall are significantly more than those based on linear calculation.

- Most of the previous major works ignored the convective portion of the pressures, and the few ones that proposed the formulations for calculation of this part of pressures is found to be underestimated and not accurate.

- Nonlinear analysis reveals that the corner sloshing will occur even under single component of earthquake load. The negative and positive maximum sloshing height is not equal in opposite sides a rectangular tank.

- The maximum sloshing height of liquid in nonlinear analysis is importantly higher that the values calculated with linear approach. While the linear maximum sloshing height can cover the nonlinear profile, nonlinear maximum sloshing height excessively dominate the linear on in wider liquid tanks.

- The wave interference in multi component earthquake loads is found to be important on the value of maximum sloshing height of the liquid. While 
Northridge sloshing height dominate sloshing height produced by El-Centro in single component earthquake, by applying bidirectional component of these earthquake loads, El-Centro sloshing heights are found to be higher excessively.

- The frequency content of earthquake ground motion has an effect on the sloshing height of liquid during time history analysis of liquid storage tanks. Low and intermediate frequency seismic ground motions (Northridge and El-Centro) generated significantly higher maximum liquid sloshing height in comparison with the value from high frequency ground motion (San-Francisco). This effect on the liquid sloshing height can be seen applicable to both in middle section and corner of the liquid tanks when it is under single component of earthquake load. The tanks under bidirectional component maintain effect only on the middle sections of the tanks.

- Most of previous studies conducted on the sloshing performance of liquid storage tanks showed that the linear ratio of $\left(H_{L} / L\right)$ has an important effect on liquid sloshing height. However, nonlinear analysis shows that the linear ratio between the depth of the water and tank plan dimension is not valid for prediction of liquid sloshing height especially for shallow tanks. this can be attributed to significance of nonlinear sloshing in shallow tanks.

- The linear formulation of ACI 350.3-06 for calculation of freeboard length is compared with the nonlinear maximum liquid sloshing height. It is founded that the function $\left(\tanh \left(3.16 H_{L} / L\right)\right)$ is not capable of estimationg the maximum sloshing height. The tank plan dimension is importantly ignored in this approach.

- The calculated results based on ACI 350.3-06 significantly underestimate the maximum liquid sloshing height and convective pressure acting on the top of tank walls.

- Nonlinear analysis is found to be more effective in wider tanks. The unique effects of nonlinearity in simulation are founded to be more critical in shallow tanks. 


\subsection{Future Studies}

A limited study was carried out as described in this thesis. Further the nonlinear analysis considering wall flexibility and soil flexibility can be carried out

The linear approach for calculation of convective term of hydrodynamic performance of liquid storage lacks accuracy. For providing better understanding of behavior of this portion of liquid domain, an experimental work is recommended, and also several liquid storage tanks could be simulated in order to introduce an accurate formulation for predicting the nonlinear behavior of convective part of retained liquid.

The maximum sloshing height in rectangular tanks occurs at the corner. As a further study, the response of rectangular liquid tanks with an optimum curve or trim at the corner of the tanks could be evaluated. Provided that an optimum curve or a trim could effectively decrease the liquid sloshing at the tank corner, liquid tanks with such details could be so favorable.

The pressure acting on the roof of the tank could be investigated as a further study. At this point of time there is no trustable calculation for this type of hydrodynamic pressure. 


\section{References}

Abaqus, (2016). V2016 User's Manual, Dassault Systems, Providence, RI, USA.

ACI 350.3-06, (2006). Seismic design of liquid-containing concrete structures (ACI 350.3-06)

and commentary (350.3R-06), American Concrete Institute (ACI) Committee 350, Environmental Engineering Concrete Structures, Farmington Hills, Mich.

Akyildi, H. Unal, E. (2006). Sloshing in a three-dimensional rectangular tank: Numerical simulation and experimental validation, Ocean Eng, 33, 2135-2149.

Akyildiz, H. and Unal, E. (2005). Experimental investigation of pressure distribution on a rectangular tank due to the liquid sloshing, Ocean Engineering, vol. 32, no. 11-12, pp. 15031516.

American Society of Civil Engineering (ASCE), (2005). Minimum design loads for buildings and other structures, ASCE Standard ASCE/SEI 7-05.

Batchelor, G.K. (1970). An introduction to fluid dynamics, Cambridge at the University Press, p. 615.

Berger, M. J., \& Colella, P. (1989). Local adaptive mesh refinement for shock hydrodynamics. Journal of Computational Physics, 82(1), 64-84.

Chen, J.Z., and Kianoush, M.R. (2005). Seismic response of concrete rectangular tanks for liquid containing structures, Canadian Journal of Civil Engineering, 32: 739-752.

Chopra, A.K. and Liaw, C.Y. (1975). Earthquake resistant design of intake-outlet towers. Journal of structural division, 101(7): 1349-136.

Choun, Y.S. and Yun, C.B., (1999). Sloshing analysis of rectangular tanks with a submerged structure by using small-amplitude water wave theory, Earthquake Engng. Struct. Dyn. 28, 763783.

Colagrossi, A., Landrini, M. (2003). Numerical simulation of interfacial flows by smoothed particle hydrodynamics, J. of Comp. Phys., 191, p. 448-475. 
Courant, R. Friedrichs, K. and Lewy, H. (1928) On the partial difference equations of mathematical physics, IBM Journal, March, 1967, pp. 215-234. English translation of a paper originally published in Mathematische Annalen 100, 32-74, 1928.

Epstein, H.I. (1976). Seismic design of liquid-storage tanks, Journal of the Structural Division, 102(9): 1659-167.

Ghaemmaghami, A.R. (2010). Dynamic time-history response of concrete rectangular liquid storage tanks (PhD thesis, Department of civil engineering. Ryerson University, Toronto, Ontario, Canada.

Ghaemmaghami, A.R. and Kianoush, M.R. (2010). Effect of wall flexibility on dynamic response of concrete rectangular tanks under horizontal and vertical ground motions. ASCE Journal of Structural Engineering, 136:4, 441-450.

Kianoush, M.R. and Ghaemmaghami, A.R. (2011). The effect of earthquake frequency content on the seismic behavior of concrete rectangular tanks using the finite element method incorporating soil-structure interaction.

Gingold R.A., Monaghan J.J. (1977). Smoothed particle hydrodynamics: theory and application to nonspherical stars, Monthly Notices of the Royal Astronomical Society, 181, 375-89.

Goudarzi, M. A., Sabbagh-Yazdi, S. R. (2012). Investigation of nonlinear sloshing effects in seismically excited tanks, Soil Dynamics and Earthquake Engineering, 43, 355-365.

Gonzales L.M., Sanchez J.M, Macia F., Souto-Iglesias A. (2009). Analysis of WCSPH laminar viscosity models, Proc. of the 4th International SPHERIC Workshop. Nantes, France, May 2729, 180-187.

Hanson, R.D. (1973). Behavior of liquid-storage tanks, in The Great Alaska earthquake of 1964: Engineering, National Academy of Science, Washington D.C., 331-339.

Haroun, M.A., (1979). Dynamic analysis of liquid storage tank, Ph.D. Thesis, Calif. Inst. Of Tech., 367-390.

Haroun, M.A. and Housner, G.W. (1981). Earthquake response of deformable liquid storage tanks, Journal of Applied Mechanics, ASME, 48, 411-418. 
Haroun, M.A. (1983). Vibration studies and tests of liquid storage tanks. Earthquake Engineering and Structural Dynamics, 11, 190-206.

Haroun, M.A. (1984). Stress analysis of rectangular walls under seismically induced hydrodynamic loads. bulletin of the seismological society of america, 74(3), 1031-1041.

Haroun, M.A. and Ellaithy, M.H. (1985). Seismically induced fluid forced on elevated tanks, Journal of Technical Topics in Civil Engineering, 111,1-15

Haroun, M.A. and Tayel, M.A. (1985). Response of tanks to vertical seismic excitations, Earthquake Engineering and Structural Dynamics, 13, 583-595.

Haroun, M.A. and Abou-Izzeddine, W. (1992). Parametric study of seismicsoil-tank interaction. I: horizontal excitation, ASCE Journal of Structural Engineering, 118:3, 783-797.

Hoskins, L.M. and Jacobsen, L.S. (1934). Water pressure in tank caused by a simulated earthquake, Bulletin of the seismological society of America, 24:1, 21-34.

Housner, G.W. (1957). Dynamic pressure on accelerated fluid containers, Bulletin of the seismological society of America, 47:1, 15-37.

Housner, G.W. (1963). The dynamic behavior of water tanks, Bulletin of the Seismological Society of America, 53:2, 381-387.

Jacobsen, L. S. (1949). Impulsive hydrodynamics of fluid Inside a cylindrical tank and of a fluid surrounding a cylindrical pier, Bull. Seism. Soc. Am., Vol. 39, 1949.

Kianoush, M.R., and Chen, J.Z. (2006). Effect of vertical acceleration on response of concrete rectangular liquid storage tanks, Engineering Structures, 28:5, 704-715.

Kianoush, M.R., Mirzabozorg, H. and Ghaemian, M. (2006). Dynamic analysis of rectangular liquid containersin three-dimensional space, Canadian Journal of Civil Engineering, 33:501-507

Kim, J.K., Koh, H.M. and Kwahk, I.J. (1996). Dynamic response of rectangular flexible fluid containers, ASCE Journal of Engineering Mechanics, 122:9, 807-817. 
Koh, H.M. Kim, J.K. and Park, J.H. (1998). Fluid-structure interaction analysis of 3D rectangular tanks by a variationally coupled BEM-FEM and comparison with test results, Earthquake Engineering and Structural Dynamics, 27, 109-124.

Liu, G.R. Liu, M.B. (2003). Smoothed particle hydrodynamics, A meshfree particle method, World Scientific Publishing Co Pte Ltd, p. 449.

Liu, M.B. Liu, G.R. Lam, K.Y. (2003). Constructing smoothing functions in smoothed particle hydrodynamics with applications, Journal of Computational and Applied Mathematics, Vol. 155, 263-284.

Liu, G.R. and Liu, M.B. (2003). Smoothed particle hydrodynamics: a meshfree particle method, Singapore: World Scientific.

Lucy, L. B. (1977). Astron. J. 82:1013.

Livaoglu, R. (2008). Investigation of seismic behavior of fluid-rectangular tank-soil/foundation systems in frequency domain, Soil Dynamics and Earthquake Engineering, 28:2, 132-146

Manos, G. C. and Clough, R. W. (1982). Further study of the earthquake response of a broad cylindrical liquid-storage tank model (UCB/EERC-82/07), Earthquake Engineering Research Center, University of California Berkeley, Berkeley.

Marchaj, T.J. (1979). Importance of vertical acceleration in the design of liquid containing tanks. In 2nd U.S. National Conference on Earthquake Engineering. Stanford, CA.

Mohammad Ali Goudarzi, Saeed Reza Sabbagh-Yazdi, (2012). Investigation of nonlinear sloshing effects in seismically excited tanks, Soil Dynamics and Earthquake Engineering, 43, $355-36$

Monaghan, J.J. (1989). On the problem of penetration in particle methods, J. of Comp. Phys. Vol. 82, 1-15.

Monaghan, J.J. (1985). Particle methods for hydrodynamics, Comput. Phys. Rep. 1985. Vol. 3, $71-124$.

Monaghan, J.J. (2005). Smoothed particle hydrodynamics, Rep. Prog. Phys. Vol. 68, 1703-1759. 
Pal, P. Bhattacharyya, S.K. (2010). Sloshing in partially filled liquid containers numerical and experimental study for 2D problems, J. Sound Vib. 329, 4466-4485

Panchal, V. R. and Jangid, R. S. (2008). Variable friction pendulum system for seismic isolation of liquid storage tanks, Nuclear Engineering and Design, Vol. 238, No. 6, 1304-1315

Park, J.H. Koh, H.M. and Kim, J. (1992). Liquid-structure interaction analysis by coupled boundary-element-finite-element-method in time domain. In Proceedings of the 7 th International Conference on Boundary Element Technology, Albuquerque, New Mexico, Edited by C.A.

Shepard, D. (1968). A two-dimensional interpolation function for irregularly-space data, Proc. of the 23rd ACM National Conference, New York, 27-29 August 1968, pp. 517-524.

NASA special publications. (1966). The dynamic behavior of liquid in moving containers, NASA SP-106, Washington, D.C.

Subhash, S. and Bhattachryya, S.K. (1996). Finite element analysis of fluid-structure interaction effect on liquid retaining structures due to sloshing, Computers and Structures, 59:6, 1165- 1171.

Sung, T.Y. (1953). Vibrations in semi-infinite solids due to periodic surface loading, ASTM STP, 156, 35-68.

Tavakkoli, Avval, I. (2012). Dynamic response of concrete rectangular liquid tanks in threedimensional space. Master's thesis. Department of civil engineering. Ryerson University. Toronto. Ontario.

Tavakkoli, Avval, I. Kianoush, M.R. and Ghaemmaghami, A.R. (2012). Sloshing characteristic of rectangular concrete tanks under seismic loading, Proc. 3rdInt. Struc. Speciality Conference. Edmonton, Alberta.

Veletsos, A.S. Kumar, A. (1989). Dynamic response of vertically excited liquid storage tanks, Proc. Eight World Conference on Earthquake Engineering, San Francisco, CA, VII, 453-460.

Veletsos, A.S. Tang, Y. and Tang, H.T. (1992). Dynamic response of flexible supported liquid storage tanks. ASCE Journal of Structural Engineering, 118: 264-28

Veletsos, A.S. and Tang, Y. (1986). Dynamics of vertically excited liquid storage tanks, ASCE Journal of Structural Engineering, 112:6, 1228-1246. 
Veletsos, A.S. and Yang, J.Y. (1977). Earthquake response of liquid storage tanks, advances in civil engineering through mechanics, ASCE Proceedings of the second engineering mechanics specially conference, Raleigh, NC: 1-24.

Virella, J.C. Prato, C.A. and Godoy, L.A. (2008). Linear and nonlinear 2D finite element analysis of sloshing modes and pressure in rectangular tanks subjected to horizontal harmonic motions, Journal of Sound and Vibration, 312:3, 442-460.

Vorobyev, A. (2012). A Smoothed particle hydrodynamics method for the simulation of centralized sloshing experiments, pp. 1-39.

Vorobyev, A. Kriventsev, V. Maschek, W. (2010). Analysis of central sloshing experiment using smoothed particle hydrodynamics (SPH) method (ICONE18-29805), 18th International Conference on Nuclear Engineering ICONE18, Xi'an, China, May 17 - 21.

Werner, P. W. and Sundquist, K. J. (1949). On hydrodynamic earthquake effects, Trans. Am. Geophys. Union, Vol. 30, 1949.

Westergaard, H.M. (1938). Water pressure on dams during earthquakes, American society of civil engineers. 98,418-433.

Yang, J.Y. (1976). Dynamic behavior of fluid-tank systems, PhD. thesis, Department of Civil Engineering, Rice University, Houston, Tex. 